\title{
COMPOSITE WAVELET BASES WITH EXTENDED STABILITY AND CANCELLATION PROPERTIES-EXTENDED VERSION*
}

\author{
ROB STEVENSON ${ }^{\dagger}$
}

\begin{abstract}
The efficient solution of operator equations using wavelets requires that they generate a Riesz basis for the underlying Sobolev space, and that they have cancellation properties of a sufficiently high order. Suitable biorthogonal wavelets were constructed on reference domains as the $n$-cube, which bases have been used, via a domain decomposition approach, as building blocks to construct biorthogonal wavelets on general domains or manifolds, where, in order to end up with local wavelets, biorthogonality was realized with respect to a modified $L_{2}$-scalar product. The use of this modified scalar product restricts the application of these so-called composite wavelets to problems of orders strictly larger than -1 , and, moreover, those wavelets with supports that extend to more than one patches generally have no cancellation properties. In this paper, we construct local, composite wavelets that are sufficiently close to being biorthogonal with respect to the standard $L_{2}$ scalar product, so that they generate Riesz bases for the Sobolev spaces $H^{s}$ for full range of $s$ that is allowed by the continuous gluing of functions over the patch interfaces, the properties of the primal and dual approximation spaces on the reference domain, and, in the manifold case, by the regularity of the manifold. Moreover, all these wavelets have cancellation properties of the full order induced by the approximation properties of the dual spaces on the reference domain. We illustrate our findings by a concrete realization of wavelets on a perturbed sphere.
\end{abstract}

Key words. Wavelets, Riesz bases, cancellation properties, domain decomposition, boundary integral equations.

AMS subject classifications. 46B15, 46E35, 65N55, 65T60.

1. Introduction. The use of wavelet bases for solving operator equations, as partial differential equations or (boundary) integral equations, has a number of advantages, cf. $[9,3]$. Assuming that the operator is symmetric, and, for $H$ being some Hilbert space, $H$-bounded and $H$-coercive, and that the infinite collection of, properly scaled, wavelets generates a Riesz basis for $H$, the stiffness matrix in wavelet coordinates resulting from a Ritz-Galerkin discretization is well-conditioned uniformly in its size, guaranteeing a uniform rate of convergence of an iterative method. In case of a differential operator, this stiffness matrix is not truly sparse, but has the well-known "finger structure". For multiplying with this matrix, however, one may switch to single-scale basis, with respect to which the stiffness matrix is sparse. For integral operators, the stiffness matrix with respect to both single-scale and wavelet basis is densely populated. Here the second important property of wavelets can be exploited of having vanishing moments or, more generally, cancellation properties, meaning that the integral of a wavelet against a smooth function vanishes with a certain order of the length scale of the wavelet. When, depending on the order of the operator and the order of approximation, this order of the cancellation properties is sufficiently large, the stiffness matrix with respect to the wavelet basis can be a priorily compressed to a sparse one without reducing the order of convergence, with which also for solving these integral equations a method of linear complexity is obtained $([19,10])$.

Instead of projecting the operator equation onto a fixed finite dimensional space, and then solving the resulting matrix-vector problem with an iterative method, the availability of a Riesz basis for $H$ opens an attractive alternative for approximating

*This work was supported by the Netherlands Organization for Scientific Research and by the EC-IHP project "Breaking Complexity."

${ }^{\dagger}$ Department of Mathematics, Utrecht University, P.O. Box 80.010, NL-3508 TA Utrecht, The Netherlands (stevenson@math.uu.nl). 
the solution by adaptive wavelet methods $([4,5])$. By writing this unknown solution in terms of this basis and testing the equation for all basis functions, one obtains an infinite dimensional matrix vector problem, that is equivalent to the operator equation, and that is well-posed in $\ell_{2}$-metric, meaning that it can be solved using an iterative method. In each iteration of such a method, the application of the infinite stiffness matrix to the current approximation vector has to be approximated. Here the concept of adaptivity enters; the accuracy with which a column is approximated grows with the modulus of the corresponding entry of the vector. The resulting method, extended with a so-called coarsening routine to remove small entries from the approximation vector, can be proven to be optimal in the following sense. Whenever, for a certain range of $s$, the solution is in a class of functions for which the error of the best $N$-term approximations from the wavelet basis decays like $N^{-s}$, the sequence of approximations produced by this adaptive method has the same rate of convergence, whereas the computational cost is equivalent to their support sizes. A necessary condition for this statement to be true is that the stiffness matrix is sufficiently close to a sparse matrix, which depends on the smoothness of the wavelets and, again, on the order of the cancellation properties ([22]). Recently, it has been shown that an optimal adaptive wavelet method can even be obtained without coarsening ([15]).

Aiming at the aforementioned applications, this paper deals with the construction on general $n$-dimensional domains or manifolds of wavelets that, properly scaled, generate Riesz bases for a range of Sobolev spaces, and satisfy cancellation properties of any required order. To be able to choose this order independently from the order of approximation, we will consider biorthogonal wavelets. Their construction starts with two nested sequences of approximation spaces that both satisfy Jackson and Bernstein estimates ("multiresolution analyses"). Then the primal and dual wavelets are sought as bases of the biorthogonal complements of successive approximation spaces at primal and dual side, respectively. In case the primal and dual approximation spaces can be equipped with bases of local, biorthogonal scaling functions, local primal wavelets are found by applying the biorthogonal projector onto a local basis of some complement space of two successive primal approximation spaces, in which case, under some mild additional condition, also the corresponding dual wavelets are local. Actually, for constructing only local primal wavelets, a reduced set of assumptions already suffices, which for simplicity we will ignore in this introduction. Note that in algorithms for solving operator equations, usually dual wavelets do not play any role.

Biorthogonal scaling functions have been constructed on the real line ([6]), and as adaptations of these, on the interval ([11]). By taking tensor products, one obtains biorthogonal scaling functions on the $n$-dimensional unit cube. To construct biorthogonal scaling functions and wavelets on general domains and manifolds, a domain decomposition approach has been developed by Dahmen and Schneider in [12] (see $[1,7]$ for related approaches). The domain or manifold of interest is written as a disjoint union of smooth parametric images of the unit cube. The biorthogonal scaling functions on the cube are lifted to the patches, and, assuming that the decomposition satisfies some matching condition, they are continuously connected over the interfaces. With respect to a modified $L_{2}$-scalar product, defined by ignoring the Jacobian determinants of the parametrizations in the definition of the canonical $L_{2}$-scalar product, the resulting collections of scaling functions are biorthogonal, and, as on the unit cube, wavelets, called composite wavelets, can be constructed using the biorthogonal projector. There are, however, two principal limitations related to the realization of biorthogonality with respect to the modified $L_{2}$-scalar product. First of all, wavelets 
with supports that extend to more than one patches, generally have no cancellation properties with respect to the canonical $L_{2}$-scalar product, so that results concerning matrix compression do not apply to entries involving such wavelets. Secondly, with respect to the interpretation of a wavelet as a functional using the duality pairing in terms of the canonical $L_{2}$-scalar product, generally the resulting wavelets cannot generate a Riesz basis for $H^{s}$ for $s \leq-\frac{1}{2}$, so that for operators of order $2 s \leq-1$, like the single-layer potential operator, neither optimal preconditioning results are valid, nor the adaptive wavelet method can be applied.

These limitations were already recognized by the authors in [12], and in [13], they developed an elegant approach to construct wavelets on general domains or manifolds that, properly scaled, generate Riesz bases for $H^{s}$ for in principal any $s$, and that have cancellation properties of any required order. Unfortunately, so far with this approach it seems not easy to construct wavelets that have competitive quantitative properties. A recent investigation of this approach was made in [16].

In this paper, we reconsider the approach from [12], except that, in view of the aforementioned limitations, we make use of the canonical $L_{2}$-scalar product. Although, generally, the lifted and connected scaling functions are not biorthogonal with respect to this scalar product, we can derive a general formula for the corresponding biorthogonal wavelets. Since this formula, however, involves the inverse of the matrix consisting of the $L_{2}$-scalar products between all primal and dual scaling functions, which matrix is thus generally not diagonal, these wavelet have global supports. On the other hand, this matrix is nearly diagonal so that its inverse can be well approximated by sparse matrices which gives rise to local, approximate wavelets. We derive general conditions under which, properly scaled, such approximate wavelets generate a Riesz basis for $H^{s}$ for the full range of $s$ that is allowed by the continuous gluing of the scaling functions over the interfaces, the properties of the primal and dual approximation spaces on the cube, and, in the manifold case, by the regularity of the manifold. We give three possibilities for the construction of approximate wavelets that are local, generate Riesz bases for $H^{s}$ for the aforementioned full range of $s$, and all have cancellation properties of the full order induced by the approximation properties of the dual spaces on the unit cube. Firstly, we show that the approximation of the inverse of the matrix of $L_{2}$-scalar products of primal and dual scaling functions by a suitable, fixed number of Jacobi iterations yields such approximate wavelets. In view of the relatively large supports of these wavelets, secondly, we show that away from the patch interfaces they can be replaced by the wavelets one gets by ignoring the Jacobian determinants, which are the wavelets from [12]. Thirdly, we show that also along the patch interfaces suitable approximate wavelets with smaller supports can be constructed, which, however, will involve solving some local systems. Although several proofs will be quite involved, we emphasize that the implementation of the approximate wavelets is relatively straightforward.

In $[14,21]$, we constructed wavelet bases for Lagrange finite element spaces based on a subdivision of polygonal domains into $n$-simplices. In this paper, we include the option that these finite element wavelets, or more precisely the underlying scaling functions are used as building blocks for wavelets on general (non-polygonal) domains or manifolds, where then the unit $n$-cube as reference domain should be replaced by some reference $n$-simplex.

This paper is organized as follows. In the remainder of this section we fix a few notations. In Section 2, we define the type of domains and manifolds and their parametrizations that we will consider, as well as the Sobolev spaces, that may involve 
zero order Dirichlet boundary conditions, which we are going to equip with Riesz bases. In Section 3, we collect all assumptions on the multiresolution analyses on the reference domain. The induced, continuous multiresolution analyses on the target domain or manifold are defined in Section 4. Put here in a more general framework, the main construction principles from Sections 2-4 originate from [12]. Biorthogonal space decompositions and the, generally, globally supported biorthogonal wavelets are constructed in Section 5. Sections 6 and 7, which form the main part of this paper, are devoted to the construction of local, approximate wavelets. Finally, in Section 8 we show examples of approximate wavelets on a perturbed sphere, and give some numerically computed condition numbers.

In order to avoid the repeated use of generic but unspecified constants, in this paper by $C \lesssim D$ we mean that $C$ can be bounded by a multiple of $D$, independently of parameters which $C$ and $D$ may depend on. Obviously, $C \gtrsim D$ is defined as $D \lesssim C$, and $C \approx D$ as $C \lesssim D$ and $C \gtrsim D$.

Let $H$ be a separable Hilbert space with with scalar product $\langle\cdot, \cdot\rangle$ and norm $\|\cdot\|$. For a countable collection $\Sigma$ of functions in $H$, which we formally view as a (column) vector, and for $\mathbf{c}=\left(c_{\sigma}\right)_{\sigma \in \Sigma}$ a vector of scalars, with $\mathbf{c}^{T} \Sigma$ we will mean the expansion $\sum_{\sigma \in \Sigma} c_{\sigma} \sigma$. The span of $\Sigma$ will be denoted as $\mathcal{S}(\Sigma)$. For $x \in H$, with $\langle\Sigma, x\rangle$ and $\langle x, \Sigma\rangle$ we will mean the column- and row-vectors with coefficients $\langle\sigma, x\rangle$ and $\langle x, \sigma\rangle$, $\sigma \in \Sigma$. When $\tilde{\Sigma}$ is another countable collection in $H$, with $\langle\Sigma, \tilde{\Sigma}\rangle$ we denote the matrix $(\langle\sigma, \tilde{\sigma}\rangle)_{\sigma \in \Sigma, \tilde{\sigma} \in \tilde{\Sigma}}$. For $V \subset H$ being a dense, continuously embedded Banach space, as usual we will use $\langle\cdot, \cdot\rangle$ sometimes also to denote the duality pairing $\langle\cdot, \cdot\rangle_{V \times V^{\prime}}$, which, with the aforementioned meaning, can also be applied to collections from $V$ and/or $V^{\prime}$.

On the spaces of (possibly infinite) scalar vectors or matrices, we will exclusively use the $\ell_{2}$-scalar product, $\ell_{2}$-norm or the resulting operator norm, that we therefore simply denote by $\langle\cdot, \cdot\rangle$ or $\|\cdot\|$, respectively. A collection $\Sigma$ is called a Riesz system when $\left\|\mathbf{c}^{T} \Sigma\right\| \approx\|\mathbf{c}\|$, i.e., when $\langle\Sigma, \Sigma\rangle$ is boundedly invertible, and $\Sigma$ is called a Riesz basis when it is in addition a basis for $H$. When $\Sigma$ depends on a parameter, we will speak about uniform Riesz systems (or bases) when the above equivalence holds uniformly over the values this parameter may attain. We set $\|\Sigma\|=\|\langle\Sigma, \Sigma\rangle\|^{\frac{1}{2}}$, and collect a few properties related to this definition:

Proposition 1.1.

(i) $\sup _{\mathbf{c} \neq 0} \frac{\left\|\mathbf{c}^{T} \Sigma\right\|}{\|\mathbf{c}\|}=\|\Sigma\|$,

(ii) $\|\langle\Sigma, \tilde{\Sigma}\rangle\| \leq\|\Sigma\|\|\tilde{\Sigma}\|$,

(iii) $\|\Sigma+\tilde{\Sigma}\| \leq\|\Sigma\|+\|\tilde{\Sigma}\|$,

(iv) For a matrix $\mathbf{A},\|\mathbf{A} \Sigma\| \leq\|\mathbf{A}\|\|\Sigma\|$.

Proof. For (i), use $\left\|\mathbf{c}^{T} \Sigma\right\|^{2}=\langle\langle\Sigma, \Sigma\rangle \mathbf{c}, \mathbf{c}\rangle$. Part (ii) follows from $|\langle\langle\Sigma, \tilde{\Sigma}\rangle \mathbf{c}, \tilde{\mathbf{c}}\rangle|=$ $\left|\left\langle\mathbf{c}^{T} \Sigma, \tilde{\mathbf{c}}^{T} \tilde{\Sigma}\right\rangle\right| \leq\|\mathbf{c}\|\|\tilde{\mathbf{c}}\|\|\Sigma\|\|\tilde{\Sigma}\|$ because of (i). Part (iii) follows easily from (ii). For (iv), use $\langle\mathbf{A} \Sigma, \mathbf{A} \Sigma\rangle=\mathbf{A}\langle\Sigma, \Sigma\rangle \mathbf{A}^{*}$.

2. Domains and function spaces. For some $n^{\prime} \geq n \geq 1$, let $\Gamma$ be an $n$ dimensional bounded manifold in $\mathbb{R}^{n^{\prime}}$, with or without a boundary. For $\square$ denoting the interior of either the $n$-cube $[0,1]^{n}$ or, despite of its notation, of some reference $n$-simplex, we assume that $\Gamma$ is given as

$$
\bar{\Gamma}=\cup_{q=1}^{M} \overline{\Gamma_{q}} \text {, with } \Gamma_{q} \cap \Gamma_{q^{\prime}}=\emptyset \text { when } q \neq q^{\prime} \text {, and } \Gamma_{q}=\kappa_{q}(\square) \text {, }
$$

where $\kappa_{q}: \mathbb{R}^{n} \rightarrow \mathbb{R}^{n^{\prime}}$ be some smooth, regular parametrizations. With $\Pi$ we will denote the collection of all affine mappings from $\square$ onto $\square$. So in case $\square$ is the interior 
of an $n$-simplex, this collection consists of the permutations of the $n+1$ barycentric coordinates, and otherwise it consists of the compositions of any permutation of the $n$ Cartesian coordinates and reflections of type $x \mapsto\left(x_{1}, \ldots, x_{i-1}, 1-x_{i}, x_{i+1}, \ldots, x_{n}\right)$ $(1 \leq i \leq n)$. We assume that the splitting of $\Gamma$ into the patches $\Gamma_{q}$ is conforming in the sense for any $q \neq q^{\prime}, \overline{\Gamma_{q}} \cap \overline{\Gamma_{q^{\prime}}}$ is either empty, or

$$
\kappa_{q}^{-1}\left(\overline{\Gamma_{q}} \cap \overline{\Gamma_{q^{\prime}}}\right) \text { is a face of } \square,
$$

and, in addition, that the parametrizations can be chosen such that the following matching condition is satisfied: There exists a $\pi \in \Pi$ with

$$
\kappa_{q^{\prime}} \circ \pi \circ \kappa_{q}^{-1}=I d \quad \text { on } \overline{\Gamma_{q}} \cap \overline{\Gamma_{q^{\prime}}} .
$$

Here and in the remainder of this paper, with a "face" of $\square$, we mean a (complete, closed) face of any dimension $0 \leq k \leq n-1$, i.e., for $n=3$, it is either a vertex, an edge or a facet. Note that our setting allows $\Gamma$ to be a bounded domain in $\mathbb{R}^{n}$, as well as an open or closed bounded manifold in $\mathbb{R}^{n^{\prime}}$ for some $n^{\prime}>n$.

We include the possibility that homogeneous, zero order Dirichlet boundary conditions are prescribed on some part $\partial \Gamma_{D} \subset \bar{\Gamma} \backslash \Gamma$, for which, for all $1 \leq q \leq M$,

$$
\kappa_{q}^{-1}\left(\partial \Gamma_{D} \cap \bar{\Gamma}_{q}\right) \text { is a, possibly empty, union of faces of } \square \text {, }
$$

see Figure 2.1.

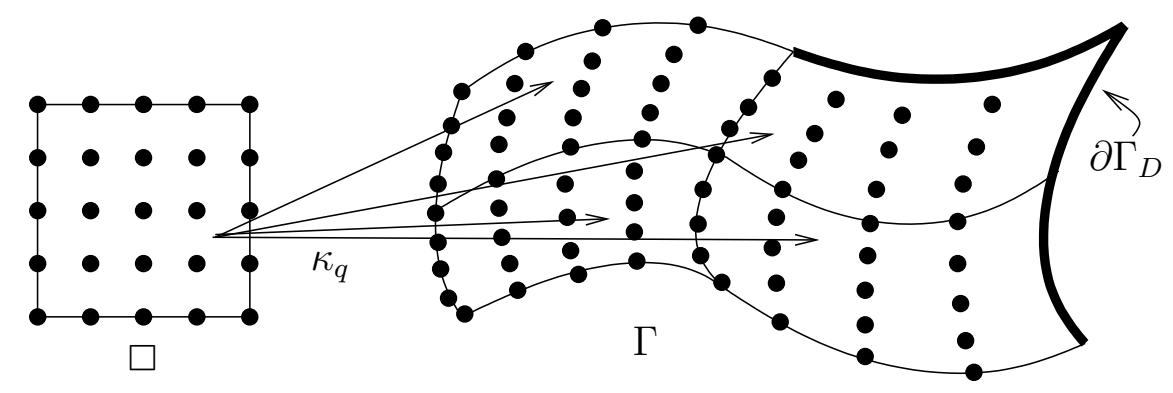

FIG. 2.1. Illustration of the domain decomposition approach

For some $s_{\Gamma}>0$, we assume that, globally,

$$
\Gamma \in C^{s_{\Gamma}} \text { when } s_{\Gamma} \notin \mathbb{N} \text {, or } \Gamma \in C^{s_{\Gamma}-1,1} \text { when } s_{\Gamma} \in \mathbb{N},
$$

which means that for $0 \leq s<s_{\Gamma} \notin \mathbb{N}$, or $0 \leq s \leq s_{\Gamma} \in \mathbb{N}$, the Sobolev spaces

$$
\mathcal{H}^{s}(\Gamma):=\left\{\begin{array}{cc}
H_{0, \partial \Gamma_{D}}^{s}(\Gamma) & \text { when } s \leq 1, \\
H^{s}(\Gamma) \cap H_{0, \partial \Gamma_{D}}^{1}(\Gamma) & \text { when } s>1
\end{array}\right.
$$

can be defined in the usual way using a partition of unity relative to some atlas. For $s>0$ in the above range, $\mathcal{H}^{-s}(\Gamma)$ will be understood as being the dual of $\mathcal{H}^{s}(\Gamma)$.

With $\mu$ being the induced Lebesgue measure on $\Gamma$, the inner product on $L_{2}(\Gamma)$ is given by

$$
\langle u, v\rangle_{L_{2}(\Gamma)}=\int_{\Gamma} u \bar{v} d \mu=\sum_{q=1}^{M}\left\langle u \circ \kappa_{q}, v \circ \kappa_{q}\right\rangle_{L_{2}(\square),\left|\partial \kappa_{q}\right|},
$$


where, for $w \in L^{\infty}(\square)$ with $w>0$ a.e., $\langle f, g\rangle_{L_{2}(\square), w}:=\int_{\square} f(z) \overline{g(z)} w(z) d z$, and $\left|\partial \kappa_{q}\right|: z \mapsto\left|\partial \kappa_{q}(z)\right|$ are the Jacobian determinants of the parametrizations. We will also make use of a modified inner product

$$
\langle\langle u, v\rangle\rangle_{0}:=\sum_{q=1}^{M}\left\langle u \circ \kappa_{q}, v \circ \kappa_{q}\right\rangle_{L_{2}(\square)},
$$

which is the inner product one gets by ignoring the Jacobian determinants, and which is equivalent to $\langle,\rangle_{L_{2}(\Gamma)}$ in the sense that $\|\cdot\|_{0}:=\langle\langle\cdot, \cdot\rangle\rangle_{0}^{\frac{1}{2}} \approx\|\cdot\|_{L_{2}(\Gamma)}$. More generally, for any $s \geq 0$, we define

$$
\langle\langle u, v\rangle\rangle_{s}=\sum_{q=1}^{M}\left\langle u \circ \kappa_{q}, v \circ \kappa_{q}\right\rangle_{H^{s}(\square)}
$$

and let $\mathcal{H}_{s}(\Gamma)$ denote the closure with respect to $\|\cdot\|_{s}:=\langle\langle\cdot, \cdot\rangle\rangle_{s}^{\frac{1}{2}}$ of the set all globally continuous, and with respect to the subdivision $\bar{\Gamma}=\cup_{q=1}^{M} \overline{\Gamma_{q}}$, piecewise $C^{\infty}$ functions on $\Gamma$ that are zero on $\partial \Gamma_{D}$, and define $\mathcal{H}_{-s}(\Gamma)=\left(\mathcal{H}_{s}(\Gamma)\right)^{\prime}$. For $0 \leq s<s_{\Gamma} \notin \mathbb{N}$ or $0 \leq s \leq s_{\Gamma} \in \mathbb{N}$, it holds that $\|\cdot\|_{H^{s}(\Gamma)} \approx\|\cdot\|_{s}$ on $H^{s}(\Gamma)$. Furthermore, if $s<\frac{3}{2}$, then the functions in the aforementioned set generate a dense subset in $\mathcal{H}^{s}(\Gamma)$. Using in addition duality, we infer that

$$
\mathcal{H}^{s}(\Gamma) \asymp \mathcal{H}_{s}(\Gamma) \quad\left(|s|<\frac{3}{2} \text { with }|s|<s_{\Gamma} \notin \mathbb{N} \text { or }|s| \leq s_{\Gamma} \in \mathbb{N}\right),
$$

meaning that both spaces agree as sets and have equivalent norms. The spaces $\mathcal{H}_{s}(\Gamma)$ will only serve as auxiliary spaces to be able to prove that the wavelets we are going to construct generate, properly scaled, a Riesz basis for $\mathcal{H}^{s}$ for the full range of $s$, in case this range is limited by the regularity of $\Gamma$ to a closed range $\left[-s_{\Gamma}, s_{\Gamma}\right]$.

3. Multiresolution analyses on the reference domain. On the reference domain, we will need two nested sequences of approximation spaces (multiresolution analyses) that satisfy Jackson and Bernstein estimates. We will assume that these spaces are equipped with single-scale bases, that satisfy certain conditions concerning their supports and symmetry (cf. assumptions $(\mathcal{L}),(\mathcal{V}),(\mathcal{S})$ ), so that after their lifting to the patches, they can be continuously connected over the interfaces. Furthermore, we will assume that the rate of best approximation from these sequences is realized by some concrete projector (cf. (J) and Proposition 3.1), with which it will be shown that the induced approximation spaces on $\Gamma$ are nested and have the same rate of approximation. We will make some assumptions ((J1) and (J2)) connecting primal and dual multiresolution analyses to ensure the existence and uniform boundedness of the biorthogonal projector (cf. Proposition 5.2), and finally we will assume the existence of a suitable "initial stable completion".

For $j \in \mathbb{N}_{0}$, let $I_{j}^{\square} \subset \bar{\square}$ be some index set with

$$
\pi\left(I_{j}^{\square}\right)=I_{j}^{\square} \quad(\pi \in \Pi), \quad \sup _{y \in \square} \#\left(I_{j}^{\square} \cap B\left(y ; 2^{-j}\right)\right) \lesssim 1
$$

(see Figure 2.1). For completeness, for $A \subset \mathbb{R}^{n}$ and $\delta \geq 0$, with $B(A ; \delta)$ we mean $\left\{x \in \mathbb{R}^{n}: \operatorname{dist}(x, A) \leq \delta\right\}$, and $B(\emptyset ; \delta):=\emptyset$. For $j \in \mathbb{N}_{0}$, we assume a collection $\Phi_{j}^{\square}=\left(\phi_{j, x}^{\square}\right)_{x \in I_{j}^{\square}} \subset C(\bar{\square})$, usually referred to as being the set of scaling functions, 
such that

$(\mathcal{L}) \exists$ constant $\varepsilon>0, \operatorname{supp} \phi_{j, x}^{\square} \subset B\left(x ; \varepsilon 2^{-j}\right)$.

$(\mathcal{V}) \phi_{j, x}^{\square}$ vanishes on any face of $\square$ that does not contain $x$.

(S) $\phi_{j, x}^{\square}=\phi_{j, \pi(x)}^{\square} \circ \pi, \quad(\pi \in \Pi)$.

(R) $\Phi_{j}^{\square}$ is a uniform $L_{2}(\square)$-Riesz system.

(J) There exists a collection of functionals $\Lambda_{j}^{\square}=\left(\lambda_{j, x}^{\square}\right)_{x \in I_{j}^{\square}} \subset C(\bar{\square})^{\prime}$ such that

(i) $\exists$ constant $\vartheta>0$, supp $\lambda_{j, x}^{\square} \subset B\left(x ; \vartheta 2^{-j}\right)$.

(ii) If $x \in \partial \square$, then supp $\lambda_{j, x}^{\square}$ is contained in the lowest dimensional face of that contains $x$.

(iii) $\left\langle u, \lambda_{j, \pi(x)}^{\square}\right\rangle_{L_{2}(\square)}=\left\langle u \circ \pi, \lambda_{j, x}^{\square}\right\rangle_{L_{2}(\square)}, \quad(\pi \in \Pi)$.

(iv) $\left|\left\langle u, \lambda_{j, x}^{\square}\right\rangle_{L_{2}(\square)}\right| \lesssim 2^{-j n / 2}\|u\|_{L_{\infty}\left(\operatorname{supp} \lambda_{j, x}^{\square}\right)}$.

(v) $\left\langle\Phi_{j}^{\square}, \Lambda_{j}^{\square}\right\rangle_{L_{2}(\square)}=I d$.

(vi) For some $\frac{n}{2}<d \in \mathbb{N}, P_{d-1}(\square) \subset \mathcal{S}\left(\Phi_{j}^{\square}\right)$.

$(\mathcal{N}) \mathcal{S}\left(\Phi_{j}^{\square}\right) \subset \mathcal{S}\left(\Phi_{j+1}^{\square}\right)$.

(B) For some $\gamma>0$, and any $s \in[0, \gamma)$, it holds that

$$
\left\|u_{j}\right\|_{H^{s}(\square)} \lesssim 2^{s j}\left\|u_{j}\right\|_{L_{2}(\square)}, \quad\left(u_{j} \in \mathcal{S}\left(\Phi_{j}^{\square}\right)\right) .
$$

Note that, in particular, (J)(ii) implies that for $x$ being a vertex of $\square,\left\langle u, \lambda_{j, x}^{\square}\right\rangle_{L_{2}(\square)}$ is a multiple of $u(x)$. Examples of such collections will be given at the end of this section. have

Proposition 3.1. For the projector $P_{j}^{\square}: u \mapsto\left\langle u, \Lambda_{j}^{\square}\right\rangle_{L_{2}(\square)} \Phi_{j}^{\square}$ onto $\mathcal{S}\left(\Phi_{j}^{\square}\right)$, we

$$
\left\|u-P_{j}^{\square} u\right\|_{L_{2}(\diamond)} \lesssim 2^{-d j}|u|_{H^{d}\left(B\left(\diamond ;(\vartheta+3 \varepsilon) 2^{-j}\right) \cap \square\right)}, \quad\left(\diamond \subset \square, u \in H^{d}(\square)\right) .
$$

Proof. Although the proof follows standard lines (cf. [12, Lemma 3.2.1]), we include it since some arguments will be used more often. Using (J)(iv) and $d>\frac{n}{2}$, by applying the Sobolev embedding theorem on a Lipschitz domain with volume of order one as well as $\|\cdot\|_{H^{d}} \approx\left[\|\cdot\|_{L_{2}}^{2}+|\cdot|_{H^{d}}^{2}\right]^{\frac{1}{2}}$ on such a domain, together with a homogeneity argument, we infer that

$$
\left|\left\langle u, \lambda_{j, y}^{\square}\right\rangle_{L_{2}(\square)}\right| \lesssim\|u\|_{L_{2}\left(B\left(y ; \vartheta 2^{-j}\right) \cap \square\right)}+2^{-d j}|u|_{H^{d}\left(B\left(y ; \vartheta 2^{-j}\right) \cap \square\right)} \cdot
$$

Since $P_{j}^{\square}$ reproduces $P_{d-1}(\square)$, for each $x \in I_{j}^{\square}$ and $p \in P_{d-1}(\square)$ we have

$$
\begin{aligned}
& \left\|u-P_{j}^{\square} u\right\|_{L_{2}\left(B\left(x ; \varepsilon 2^{-j}\right) \cap \square\right)}=\left\|u-p-P_{j}^{\square}(u-p)\right\|_{L_{2}\left(B\left(x ; \varepsilon 2^{-j}\right) \cap \square\right)} \\
& \leq\|u-p\|_{L_{2}\left(B\left(x ; \varepsilon 2^{-j}\right) \cap \square\right)}+\left\|\sum_{y \in I_{j}^{\square} \cap B\left(x ; 2 \varepsilon 2^{-j}\right)}\left\langle u-p, \lambda_{j, y}^{\square}\right\rangle_{L_{2}(\square)} \phi_{j, y}\right\|_{L_{2}\left(B\left(x ; \varepsilon 2^{-j}\right) \cap \square\right)} \\
& \lesssim\|u-p\|_{L_{2}\left(B\left(x ; \varepsilon 2^{-j}\right) \cap \square\right)}+\sum_{y \in I_{j}^{\square} \cap B\left(x ; 2 \varepsilon 2^{-j}\right)}\|u-p\|_{L_{2}\left(B\left(y ; \vartheta 2^{-j}\right) \cap \square\right)}+2^{-d j}|u|_{H^{d}\left(B\left(y ; \vartheta 2^{-j}\right) \cap \square\right)} \\
& \lesssim\|u-p\|_{L_{2}\left(B\left(x ;(\vartheta+2 \varepsilon) 2^{-j}\right) \cap \square\right)}+2^{-d j}|u|_{H^{d}\left(B\left(x ;(\vartheta+2 \varepsilon) 2^{-j}\right) \cap \square\right)},
\end{aligned}
$$


where we have used that $\sup _{z \in \square} \#\left(I_{j}^{\square} \cap B\left(z ; 2^{-j}\right)\right) \lesssim 1$. By taking the infimum over $p$, using the Bramble-Hilbert lemma we find that $\left\|u-P_{j}^{\square} u\right\|_{L_{2}\left(B\left(x ; \varepsilon 2^{-j}\right) \cap \square\right)} \lesssim$ $2^{-d j}|u|_{H^{d}\left(B\left(x ;(\vartheta+2 \varepsilon) 2^{-j}\right) \cap \square\right)}$. Since $P_{0}(\square) \subset \mathcal{S}\left(\Phi_{j}^{\square}\right),\left\{B\left(x ; \varepsilon 2^{-j}\right): x \in I_{j}^{\square}\right\}$ is a covering of $\square$, so that $\left\|u-P_{j}^{\square} u\right\|_{L_{2}(\diamond)}^{2} \leq \sum_{\left\{x \in I_{j}^{\square}: B\left(x ; \varepsilon 2^{-j}\right) \cap \diamond \neq \emptyset\right\}}\left\|u-P_{j}^{\square} u\right\|_{L_{2}\left(B\left(x ; \varepsilon 2^{-j}\right) \cap \square\right)^{2}}^{2}$. By again using that $\sup _{z \in \square} \#\left(I_{j}^{\square} \cap B\left(z ; 2^{-j}\right)\right) \lesssim 1$, we conclude the statement of the proposition.

Remark 3.2. If, when $p \in(2, \infty]$ we assume that $\left\|\phi_{j, x}\right\|_{L_{p}(\square)} \lesssim 2^{\left(\frac{n}{2}-\frac{n}{p}\right) j}$, and, when $p \in[1,2)$ that $d>\frac{n}{p}$, then an easy adaptation of the above proof shows that

$$
\left\|u-P_{j}^{\square} u\right\|_{L_{p}(\diamond)} \lesssim 2^{-d j}|u|_{W_{p}^{d}\left(B\left(\diamond ;(\vartheta+3 \varepsilon) 2^{-j}\right) \cap \square\right)}, \quad\left(\diamond \subset \square, u \in W_{p}^{d}(\square)\right) .
$$

Apart from the above collection $\Phi_{j}^{\square}$ of primal scaling functions, for $j \in \mathbb{N}_{0}$ we assume the existence of a collection $\tilde{\Phi}_{j}^{\square}=\left(\tilde{\phi}_{j, x}^{\square}\right)_{x \in I_{j}^{\square}} \subset C(\bar{\square})$ of dual scaling functions that also satisfies all of $(\mathcal{L})-(\mathcal{B})$ with the same index set $I_{j}^{\square}$, but with generally different parameters and functionals in $(\mathcal{B})$ and $(\mathcal{J})$ that we will denote as $\tilde{\gamma}>0$, $\tilde{d}>\frac{n}{2}, \tilde{\Lambda}_{j}^{\square}$, and $\tilde{\varepsilon}, \tilde{\vartheta}>0$. The resulting projector $\tilde{P}_{j}^{\square}: u \mapsto\left\langle u, \tilde{\Lambda}_{j}^{\square}\right\rangle_{L_{2}(\square)} \tilde{\Phi}_{j}^{\square}$ satisfies the analogue of Proposition 3.1 with $(d, \vartheta, \varepsilon)$ replaced by $(\tilde{d}, \tilde{\vartheta}, \tilde{\varepsilon})$.

Since $\Phi_{j}^{\square}$ and $\tilde{\Phi}_{j}^{\square}$ are uniform $L_{2}(\square)$-Riesz systems, the matrix $\left\langle\Phi_{j}^{\square}, \tilde{\Phi}_{j}^{\square}\right\rangle_{L_{2}(\square)}$ defines a uniformly bounded linear operator on $\ell_{2}\left(I_{j}^{\square}\right)$. A relation between $\mathcal{S}\left(\Phi_{j}^{\square}\right)$ and $\mathcal{S}\left(\tilde{\Phi}_{j}^{\square}\right)$ is established by assuming that that its real part satisfies

$$
\Re\left\langle\Phi_{j}^{\square}, \tilde{\Phi}_{j}^{\square}\right\rangle_{L_{2}(\square)} \gtrsim I d
$$

Finally, for $j \in \mathbb{N}_{0}$, let $J_{j}^{\square} \subset \bar{\square}$ be some index set with $\pi\left(J_{j}^{\square}\right)=J_{j}^{\square}(\pi \in \Pi)$, $\sup _{y \in \square} \#\left(J_{j}^{\square} \cap B\left(y ; 2^{-j}\right)\right) \lesssim 1$, and for $e$ being either $\square$ or any face of $\square, \#\left(\left(I_{j}^{\square} \cup\right.\right.$ $\left.\left.J_{j}^{\square}\right) \cap e\right)=\#\left(I_{j+1}^{\square} \cap e\right)$. In case $I_{j}^{\square} \subset I_{j+1}^{\square}$, a natural candidate is $J_{j}^{\square}=I_{j+1}^{\square} \backslash I_{j}^{\square}$. We assume the existence of collections $\Theta_{j}^{\square}=\left(\theta_{j, x}^{\square}\right)_{x \in I_{j}^{\square}}$ with

$$
\left\langle\Theta_{j}^{\square}, \tilde{\Phi}_{j}^{\square}\right\rangle_{L_{2}(\square)}=I d
$$

and $\Xi_{j}^{\square}=\left(\xi_{j, x}^{\square}\right)_{x \in J_{j}^{\square}}$ such that the union $\Upsilon_{j+1}^{\square}:=\left[\left(\Theta_{j}^{\square}\right)^{T} \quad\left(\Xi_{j}^{\square}\right)^{T}\right]^{T}$ satisfies $(\mathcal{L})-(\mathcal{R})$, and $\mathcal{S}\left(\Upsilon_{j+1}^{\square}\right)=\mathcal{S}\left(\Phi_{j+1}^{\square}\right)$.

Remark 3.3. "Classical" wavelet constructions start with assuming biorthogonal scaling functions, i.e., $\left\langle\Phi_{j}^{\square}, \tilde{\Phi}_{j}^{\square}\right\rangle_{L_{2}(\square)}=I d$, in which case (J1) and (J2) are satisfied with $\Theta_{j}^{\square}=\Phi_{j}^{\square}$. When there is no need for locally supported dual wavelets, biorthogonality of the scaling functions can be relaxed to the conditions given here, with generally $\Theta_{j}^{\square}$ different from $\Phi_{j}^{\square}$, and in particular not contained in $\mathcal{S}\left(\Phi_{j}\right)$. For the case that $\Theta_{j}^{\square}=\Phi_{j}^{\square}$, in the literature the set $\Xi_{j}^{\square}$ is sometimes called an initial "stable" completion of $\Phi_{j}^{\square}$, that is, a completion of $\Phi_{j}^{\square}$ to a uniform $L_{2}(\square)$-Riesz basis for $\mathcal{S}\left(\Phi_{j+1}^{\square}\right)$. The wavelets to be constructed are then thought of being the target stable completion.

Remark 3.4. The condition (J2) can be further relaxed, which turned out to be useful in [21]. Instead of assuming that $\left\langle\Theta_{j}^{\square}, \tilde{\Phi}_{j}^{\square}\right\rangle_{L_{2}(\square)}$ is diagonal, more generally it is also sufficient when, for some fixed $p, I_{j}^{\square}$ is the union of disjoint sets $I_{j, 1}^{\square}, \ldots, I_{j, p}^{\square}$, 
with $\pi\left(I_{j, i}^{\square}\right)=I_{j, i}^{\square}(\pi \in \Pi, 1 \leq i \leq p)$, such that, with respect to this partitioning, $\left\langle\Theta_{j}^{\square}, \tilde{\Phi}_{j}^{\square}\right\rangle_{L_{2}(\square)}$ is a block triangular matrix, with diagonal blocks that are identity matrices. Although all results from this paper are also valid under this relaxed assumption, for ease of presentation we will stick to assumption (J2).

Because of $\mathcal{S}\left(\Phi_{j}^{\square}\right) \subset \mathcal{S}\left(\Phi_{j+1}^{\square}\right), \mathcal{S}\left(\tilde{\Phi}_{j}^{\square}\right) \subset \mathcal{S}\left(\tilde{\Phi}_{j+1}^{\square}\right)$ and $\mathcal{S}\left(\Upsilon_{j+1}^{\square}\right)=\mathcal{S}\left(\Phi_{j+1}^{\square}\right)$, it holds that $\Phi_{j}^{\square}=\left\langle\Phi_{j}^{\square}, \Lambda_{j+1}^{\square}\right\rangle_{L_{2}(\square)} \Phi_{j+1}^{\square}, \tilde{\Phi}_{j}^{\square}=\left\langle\tilde{\Phi}_{j}^{\square}, \tilde{\Lambda}_{j+1}^{\square}\right\rangle_{L_{2}(\square)} \tilde{\Phi}_{j+1}^{\square}$, and $\Upsilon_{j+1}^{\square}=$ $\left\langle\Upsilon_{j+1}^{\square}, \Lambda_{j+1}^{\square}\right\rangle_{L_{2}(\square)} \Phi_{j+1}^{\square}$, where $\left\langle\Upsilon_{j+1}^{\square}, \Lambda_{j+1}^{\square}\right\rangle_{L_{2}(\square)}$ is uniformly boundedly invertible.

LemmA 3.5. For the matrix $\mathbf{R}_{j}^{\square}$ being either $\left\langle\Phi_{j}^{\square}, \Lambda_{j+1}^{\square}\right\rangle_{L_{2}(\square)},\left\langle\tilde{\Phi}_{j}^{\square}, \tilde{\Lambda}_{j+1}^{\square}\right\rangle_{L_{2}(\square)}$, $\left\langle\Upsilon_{j+1}^{\square}, \Lambda_{j+1}^{\square}\right\rangle_{L_{2}(\square)}$ or $\left\langle\Upsilon_{j+1}^{\square}, \Lambda_{j+1}^{\square}\right\rangle_{L_{2}(\square)}^{-1}$, it holds that

(a) $\left(\mathbf{R}_{j}^{\square}\right)_{\pi(x), \pi(y)}=\left(\mathbf{R}_{j}^{\square}\right)_{x, y}(\pi \in \Pi)$.

(b) $\left(\mathbf{R}_{j}^{\square}\right)_{x, y}=0$ when $y$ is on a face of $\square$ that does not contain $x$.

Proof. Part (a) follows from the assumptions (S) or (J)(iii) for the involved collections of functions and functionals, respectively. Similarly, for the first three matrices, Part (b) follows from the assumptions $(\mathcal{V})$ or $(\mathcal{J})(\mathrm{ii})$. Now let $e$ be a face of $\square$. With respect to the partitioning of the index sets for $\Upsilon_{j+1}^{\square}$ and $\Lambda_{j+1}^{\square}$ into indices on $e$ and indices not on $e,\left\langle\Upsilon_{j+1}^{\square}, \Lambda_{j+1}^{\square}\right\rangle_{L_{2}(\square)}$ is a $2 \times 2$ upper block triangular matrix with square diagonal blocks, and thus so is its inverse, which shows (b) also for $\left\langle\Upsilon_{j+1}^{\square}, \Lambda_{j+1}^{\square}\right\rangle_{L_{2}(\square)}^{-1}$. $\square$

By our assumptions, the matrices $\left\langle\Phi_{j}^{\square}, \Lambda_{j+1}^{\square}\right\rangle_{L_{2}(\square)}$ and $\left\langle\Upsilon_{j+1}^{\square}, \Lambda_{j+1}^{\square}\right\rangle_{L_{2}(\square)}$ are uniformly local, with which we mean that only entries with indices $(x, y)$ with $|x-y| \lesssim 2^{-j}$ might be nonzero. As a consequence, for the wavelets we are going to construct, the basis transformation from wavelet- to single scale basis will be of optimal computational complexity.

For some applications, it is also essential to have a basis transformation from single scale to wavelet basis that is of optimal computational complexity. In that case, one has to assume both that

$$
\Theta_{j}^{\square}=\Phi_{j}^{\square},
$$

with which (J1) can be dropped since it is implied by (J2), and also that

$$
\left\langle\Upsilon_{j+1}^{\square}, \Lambda_{j+1}^{\square}\right\rangle_{L_{2}(\square)}^{-1} \text { is uniformly local. }
$$

Note that, for $\Theta_{j}^{\square}=\Phi_{j}^{\square},\left\langle\Upsilon_{j+1}^{\square}, \Lambda_{j+1}^{\square}\right\rangle_{L_{2}(\square)}^{-1}$ is the basis transformation from $\Phi_{j+1}^{\square}$ to the two-level basis $\Phi_{j}^{\square} \cup \Xi_{j}^{\square}$.

All conditions imposed in this section are satisfied by the collections $\Phi_{j}^{\square}, \tilde{\Phi}_{j}^{\square}, \Theta_{j}^{\square}$, $\Xi_{j}^{\square}$ underlying the finite element wavelets introduced in [14]. With this construction, $\mathcal{S}\left(\Phi_{j}^{\square}\right), \mathcal{S}\left(\tilde{\Phi}_{j}^{\square}\right)$ are standard Lagrange finite element spaces, so that $\gamma=\tilde{\gamma}=\frac{3}{2}$, of orders $d$ and $\tilde{d}$, respectively, with respect to a $j$ times repeated uniform dyadic refinement of an initial simplicial partition of a polytope. For the present setting, we take this polytope to be a reference $n$-simplex. Thinking of $\tilde{d} \geq d$, these orders are chosen such that there is an $m \in \mathbb{N}$ with $2^{m}(d-1)=\tilde{d}-1$, so that with the initial partition at the dual side being the reference simplex itself, and at the primal side being created by applying $m$ dyadic recursive refinements to this simplex, we have $\operatorname{dim} \mathcal{S}\left(\Phi_{j}^{\square}\right)=\operatorname{dim} \mathcal{S}\left(\tilde{\Phi}_{j}^{\square}\right)$. So each "element" at the dual side is equal to a macroelement at the primal side consisting of $2^{m}$ "elements". The collections $\tilde{\Phi}_{j}^{\square}$ at the dual side, and $\Phi_{j}^{\square}, \Theta_{j}^{\square}, \Xi_{j}^{\square}$ at the primal side are now assembled in the standard 
finite element fashion from local collections, of a small, fixed dimension, living on the individual elements or macro-elements, respectively, each of these collections being a copy, or more precisely, a push-forward using an affine bijection of such a collection created once and for all on a reference (macro-) element. The functionals from $\tilde{\Lambda}_{j}^{\square}$ and $\Lambda_{j}^{\square}$ are assembled in the same manner from local collections, and are either simply scaled function evaluations in the "nodal points" $I_{j}^{\square}$, or local linear combinations of these. Actually, in the present paper, we will repeat the idea of assembling functions and functionals from collections defined on (macro-) elements, which in turn are pushforwards of collections defined on a reference (macro-) element, where the role of the (macro-) elements will now be played by the patches $\Gamma_{q}$, and that of the reference (macro-) element by $\kappa_{q}^{-1}\left(\Gamma_{q}\right)$. A difference is that the number of patches is fixed, and that, as a consequence, the dimension of the local collections grows with the level. The major difficulty we have to deal with is that generally the $\kappa_{q}$ are not affine, so that the Jacobian determinants are not constants.

In [21], we reconsidered the finite element wavelets, and constructed collections with $\Theta_{j}^{\square}=\Phi_{j}^{\square}$, so that also the resulting dual wavelets are locally supported. In this case, the dual spaces, although consisting of continuous piecewise polynomials, are not standard finite element spaces.

Other examples of collections $\Phi_{j}^{\square}, \tilde{\Phi}_{j}^{\square}, \Theta_{j}^{\square}, \Xi_{j}^{\square}$ satisfying our assumptions, with $\Theta_{j}^{\square}=\Phi_{j}^{\square}$, and with $\square$ now being the $n$-cube, are given in [12], which underly the construction of biorthogonal spline wavelets. These collections are slight modifications of those developed in [11], and, for $n>1$, they are simply generated using tensor products from univariate collections $\Phi_{j}^{[0,1]}, \tilde{\Phi}_{j}^{[0,1]}, \Xi_{j}^{[0,1]}$ defined on $[0,1]$. For given $\tilde{d} \geq d \geq 2$ with $d+\tilde{d}$ even, $\mathcal{S}\left(\Phi_{j}^{[0,1]}\right)$ is the spline space of order $d$, so that $\gamma=d-\frac{1}{2}$, with respect to the knot sequence

$$
(\underbrace{0, \ldots, 0}_{d \text { times }}, r 2^{-j}, r 2^{-j}+2^{-j}, \ldots, 1-r 2^{-j}, \underbrace{1, \ldots, 1}_{d \text { times }}) .
$$

where $\mathbb{N} \ni r \geq d-1$ is some parameter that one can choose. The collection $\tilde{\Phi}_{j}^{[0,1]}$ is such that $P_{\tilde{d}-1}[0,1] \subset \mathcal{S}\left(\tilde{\Phi}_{j}^{[0,1]}\right)$ and $\left\langle\Phi_{j}^{[0,1]}, \tilde{\Phi}_{j}^{[0,1]}\right\rangle_{L_{2}([0,1])}=I d$, where $\tilde{\gamma}$ grows linearly with $\tilde{d}$. For $x$ not near the endpoints 0 or $1, \phi_{j, x}^{[0,1]}=2^{j / 2} \phi\left(2^{j} \cdot-x\right)$ and $\tilde{\phi}_{j, x}^{[0,1]}=2^{j / 2} \tilde{\phi}\left(2^{j} \cdot-x\right)$, where $(\phi, \tilde{\phi})$ is a biorthogonal pair constructed in [6]. Also the functionals from $\Lambda_{j}^{\square}$ and $\tilde{\Lambda}_{j}^{\square}$ are constructed from the collections of univariate functionals $\Lambda_{j}^{[0,1]}$ and $\tilde{\Lambda}_{j}^{[0,1]}$ using tensor products, where $\lambda_{j, x}^{[0,1]}=\tilde{\phi}_{j, x}^{[0,1]}, \tilde{\lambda}_{j, x}^{[0,1]}=\phi_{j, x}^{[0,1]}$ for $x \notin\{0,1\}$, and where they are simply scaled function evaluations in 0 or 1 , respectively, otherwise.

4. Induced, continuous multiresolution analyses on $\Gamma$. By lifting the collections of functions on $\square$ to the patches of $\Gamma$, and by connecting those that do not vanish at the interfaces continuously with ones from other patches, we will construct nested sequences of primal and dual spaces that satisfy Jackson estimates and Bernstein inequalities.

We define the index sets $I_{j} \subset \bar{\Gamma} \backslash \partial \Gamma_{D}$, and analogously $J_{j}$, by

$$
I_{j}=\left(\cup_{q=1}^{M} \kappa_{q}\left(I_{j}^{\square}\right)\right) \cap\left(\bar{\Gamma} \backslash \partial \Gamma_{D}\right)
$$

(see Figure 2.1). By $(\mathcal{M})$ and $\pi\left(I_{j}^{\square}\right)=I_{j}^{\square}(\pi \in \Pi)$, for any $1 \leq q, q^{\prime} \leq M$ with 
$\overline{\Gamma_{q}} \cap \overline{\Gamma_{q^{\prime}}} \neq \emptyset$, the sets $\kappa_{q}\left(I_{j}^{\square}\right)$ and $\kappa_{q^{\prime}}\left(I_{j}^{\square}\right)$ restricted to this interface coincide. For $x \in \bar{\Gamma}$, we set $k(x)=\#\left\{q: x \in \overline{\Gamma_{q}}\right\}$.

For $j \in \mathbb{N}_{0}$, we define the collection $\Phi_{j}=\left(\phi_{j, x}\right)_{x \in I_{j}} \subset C(\bar{\Gamma})$ by

$$
\phi_{j, x}(y)=k(x)^{-\frac{1}{2}}\left\{\begin{array}{cl}
\phi_{j, \kappa_{q}^{-1}(x)}^{\square}\left(\kappa_{q}^{-1}(y)\right) & \text { when } x, y \in \overline{\Gamma_{q}} \text { for some } 1 \leq q \leq M, \\
0 & \text { elsewhere. }
\end{array}\right.
$$

Note that by $(\mathcal{S}),(\mathcal{V})$ and $(2.1), \phi_{j, x}$ is well-defined, indeed continuous and it vanishes on $\partial \Gamma_{D}$. By assumption $(\mathcal{L})$, the collection $\Phi_{j}$ is uniformly local, with which we mean that $x \in \operatorname{supp} \phi_{j, x}$, and that $d_{\Gamma}(x, y) \lesssim 2^{-j}$ for any $y \in \operatorname{supp} \phi_{j, x}$, where $d_{\Gamma}(x, y)$ denotes the geodesic distance of $x$ and $y$ over $\Gamma$, i.e., the length of the shortest curve on $\Gamma$ connecting $x$ and $y$.

With $\mathbf{E}_{j, q}: \ell_{2}\left(I_{j}^{\square}\right) \rightarrow \ell_{2}\left(I_{j}\right)$ defined by

$$
\left(\mathbf{E}_{j, q} \mathbf{c}_{j}^{\square}\right)_{x}=k(x)^{-\frac{1}{2}}\left\{\begin{array}{cl}
\mathbf{c}_{j, \kappa_{q}^{-1}(x)}^{\square} & x \in \overline{\Gamma_{q}}, \\
0 & \text { otherwise, }
\end{array}\right.
$$

and similarly $\mathbf{F}_{j, q}: \ell_{2}\left(J_{j}^{\square}\right) \rightarrow \ell_{2}\left(J_{j}\right)$, we have $\sum_{q=1}^{M} \mathbf{E}_{j, q} \mathbf{E}_{j, q}^{T}=I d$ and $\sum_{q=1}^{M} \mathbf{F}_{j, q} \mathbf{F}_{j, q}^{T}=$ $I d$. By construction of $\Phi_{j}$ from $\Phi_{j}^{\square}$, we have

$$
\left\langle\Phi_{j}, \Phi_{j}\right\rangle_{L_{2}(\Gamma)}=\sum_{q=1}^{M} \mathbf{E}_{j, q}\left\langle\Phi_{j}^{\square}, \Phi_{j}^{\square}\right\rangle_{L_{2}(\square), \mid \partial \kappa_{q}} \mathbf{E}_{j, q}^{T},
$$

so that, because of $\left\langle\Phi_{j}^{\square}, \Phi_{j}^{\square}\right\rangle_{L_{2}(\square),\left|\partial \kappa_{q}\right|} \bar{\sim}\left\langle\Phi_{j}^{\square}, \Phi_{j}^{\square}\right\rangle_{L_{2}(\square)} \approx I d$ by $\left|\partial \kappa_{q}\right| \approx 1$ and (R), $\Phi_{j}$ is a uniform $L_{2}(\Gamma)$-Riesz system.

Proposition 4.1. Setting $\Lambda_{j}=\left(\lambda_{j, x}\right)_{x \in I_{j}} \subset C(\bar{\Gamma})^{\prime}$ by

$$
\lambda_{j, x}(u)=k(x)^{\frac{1}{2}} \lambda_{j, \kappa_{q}^{-1}(x)}^{\square}\left(u \circ \kappa_{q}\right) \quad \text { when } x \in \bar{\Gamma}_{q},
$$

we have $\left\langle\Phi_{j}, \Lambda_{j}\right\rangle_{L_{2}(\Gamma)}=I d$. The projector $P_{j}: u \mapsto\left\langle u, \Lambda_{j}\right\rangle_{L_{2}(\Gamma)} \Phi_{j}$ onto $\mathcal{S}\left(\Phi_{j}\right)$ satisfies

$\left\|\left(I d-P_{j}\right) u\right\|_{L_{2}(\Omega)} \lesssim 2^{-d j} \sum_{q=1}^{M}\left|u \circ \kappa_{q}\right|_{H^{d}\left(B\left(\kappa_{q}^{-1}\left(\Omega \cap \Gamma_{q}\right) ;(\vartheta+3 \varepsilon) 2^{-j}\right) \cap \square\right)} \quad\left(\Omega \subset \Gamma, u \in \mathcal{H}_{d}(\Gamma)\right)$.

Proof. Assumption (J)(ii) shows that, for $u \in C(\bar{\Gamma}), \lambda_{j, x}(u)$ is well-defined, also when $x$ is on an interface between patches, and, because of $(4.1)$, that $\left(P_{j} u\right) \circ \kappa_{q}=$ $P_{j}^{\square}\left(u \circ \kappa_{q}\right)$ when $u$ vanishes on $\partial \Gamma_{D}$. Condition $(\mathcal{J})(\mathrm{v})$ shows that $\left\langle\Phi_{j}, \Lambda_{j}\right\rangle_{L_{2}(\Gamma)}=I d$. By Proposition 3.1, we have

$$
\begin{aligned}
& \left\|\left(I d-P_{j}\right) u\right\|_{L_{2}(\Omega)} \bar{\sim} \sum_{q=1}^{M}\left\|\left(\left(I d-P_{j}\right) u\right) \circ \kappa_{q}\right\|_{L_{2}\left(\kappa_{q}^{-1}\left(\Omega \cap \Gamma_{q}\right)\right)}= \\
& \sum_{q=1}^{M}\left\|\left(I d-P_{j}^{\square}\right)\left(u \circ \kappa_{q}\right)\right\|_{L_{2}\left(\kappa_{q}^{-1}\left(\Omega \cap \Gamma_{q}\right)\right)} \lesssim 2^{-d j} \sum_{q=1}^{M}\left|u \circ \kappa_{q}\right|_{H^{d}\left(B\left(\kappa_{q}^{-1}\left(\Omega \cap \Gamma_{q}\right) ;(\vartheta+3 \varepsilon) 2^{-j}\right) \cap \square\right)} .
\end{aligned}
$$

By substituting $\Omega=\Gamma$ in Proposition 4.1, we have the following Jackson estimate:

$$
\inf _{u_{j} \in \mathcal{S}\left(\Phi_{j}\right)}\left\|u-u_{j}\right\|_{L_{2}(\Gamma)} \lesssim 2^{-d j}\|u\|_{d}, \quad\left(u \in \mathcal{H}_{d}(\Gamma)\right) .
$$


A direct consequence of $(\mathcal{B})$ is the following Bernstein inequality: For $s \in[0, \gamma)$,

$$
\left\|u_{j}\right\|_{s} \lesssim 2^{s j}\left\|u_{j}\right\|_{L_{2}(\Gamma)}, \quad\left(u_{j} \in \mathcal{S}\left(\Phi_{j}\right)\right) .
$$

Thanks to properties of a Sobolev scale, (4.5) gives rise to the following extended version that will be used in the appendix:

LEMmA 4.2. For any $t \leq s<\gamma$ with $t \leq 0$,

$$
\left\|u_{j}\right\|_{s} \lesssim 2^{(s-t) j}\left\|u_{j}\right\|_{t}, \quad\left(u_{j} \in \mathcal{S}\left(\Phi_{j}\right)\right) .
$$

Proof. For $s=0$, the statement follows from $\|\cdot\|_{0} \lesssim\|\cdot\|_{t}^{\frac{\gamma^{\prime}}{\gamma^{\prime}-t}}\|\cdot\|_{\gamma^{\prime}}^{\frac{-t}{\gamma^{\prime}-t}}$ on $\mathcal{H}_{\gamma^{\prime}}(\Gamma)$ for some $\gamma^{\prime} \in(0, \gamma)$, and $\|\cdot\|_{\gamma^{\prime}} \lesssim 2^{\gamma^{\prime} j}\|\cdot\|_{0}$ on $\mathcal{S}\left(\Phi_{j}\right)$. An additional application of $\|\cdot\|_{s} \lesssim 2^{s j}\|\cdot\|_{0}$ on $\mathcal{S}\left(\Phi_{j}\right)$ when $s>0$, or $\|\cdot\|_{s} \lesssim\|\cdot\|_{0}^{\frac{-t+s}{-t}}\|\cdot\|_{t}^{\frac{-s}{-t}}$ on $L_{2}(\Gamma)$ when $s<0$ shows it for any $s \in[t, \gamma)$. $\square$

As $\Phi_{j}^{\square}$, via (4.1), gave rise to a uniformly local, uniform $L_{2}(\Gamma)$-Riesz system $\Phi_{j}$, analogously the collections $\Upsilon_{j+1}^{\square}=\left[\begin{array}{lll}\left(\Theta_{j}^{\square}\right)^{T} & \left(\Xi_{j}^{\square}\right)^{T}\end{array}\right]^{T}$ and $\tilde{\Phi}_{j}^{\square}$ yield uniformly local, uniform $L_{2}(\Gamma)$-Riesz systems $\Upsilon_{j+1}=\left[\begin{array}{ll}\Theta_{j}^{T} & \Xi_{j}^{T}\end{array}\right]^{T}$ and $\tilde{\Phi}_{j}$, respectively.

We have the analogue of Proposition 4.1 at the dual side, with functionals and a projector denoted as $\tilde{\Lambda}_{j}=\left(\tilde{\lambda}_{j, x}\right)_{x \in I_{j}}$ and $\tilde{P}_{j}$, respectively, and with $\Phi_{j}, d, \vartheta, \varepsilon$ replaced by $\tilde{\Phi}_{j}, \tilde{d}, \tilde{\vartheta}, \tilde{\varepsilon}$. In particular, we have the Jackson estimate

$$
\inf _{u_{j} \in \mathcal{S}\left(\tilde{\Phi}_{j}\right)}\left\|u-u_{j}\right\|_{L_{2}(\Gamma)} \lesssim 2^{-\tilde{d} j}\|u\|_{\tilde{d}}, \quad\left(u \in \mathcal{H}_{\tilde{d}}(\Gamma)\right),
$$

and furthermore also the Bernstein inequality: For any $s \in[0, \tilde{\gamma})$,

$$
\left\|u_{j}\right\|_{s} \lesssim 2^{s j}\left\|u_{j}\right\|_{L_{2}(\Gamma)}, \quad\left(u_{j} \in \mathcal{S}\left(\tilde{\Phi}_{j}\right)\right),
$$

which can be extended analogously to Lemma 4.2 .

Analogously to [12, Prop. 4.3.1], using Lemma 3.5, one may verify the following easily implementable formulas for the representations of the global embeddings in terms of corresponding representations of local embeddings.

Proposition 4.3. It holds that

$$
\begin{aligned}
\Phi_{j} & =\sum_{q=1}^{M} \mathbf{E}_{j, q}\left\langle\Phi_{j}^{\square}, \Lambda_{j+1}^{\square}\right\rangle_{L_{2}(\square)} \mathbf{E}_{j+1, q}^{T} \Phi_{j+1}, \\
\tilde{\Phi}_{j} & =\sum_{q=1}^{M} \mathbf{E}_{j, q}\left\langle\tilde{\Phi}_{j}^{\square}, \tilde{\Lambda}_{j+1}^{\square}\right\rangle_{L_{2}(\square)} \mathbf{E}_{j+1, q}^{T} \tilde{\Phi}_{j+1}, \\
\Upsilon_{j+1} & =\sum_{q=1}^{M}\left[\begin{array}{l|l}
\mathbf{E}_{j, q} & 0 \\
\hline 0 & \mathbf{F}_{j, q}
\end{array}\right]\left\langle\Upsilon_{j+1}^{\square}, \Lambda_{j+1}^{\square}\right\rangle_{L_{2}(\square)} \mathbf{E}_{j+1, q}^{T} \Phi_{j+1}, \\
\Phi_{j+1} & =\sum_{q=1}^{M} \mathbf{E}_{j+1, q}\left\langle\Upsilon_{j+1}^{\square}, \Lambda_{j+1}^{\square}\right\rangle_{L_{2}(\square)}^{-1}\left[\begin{array}{c|c}
\mathbf{E}_{j, q}^{T} & 0 \\
\hline 0 & \mathbf{F}_{j, q}^{T}
\end{array}\right] \Upsilon_{j+1} .
\end{aligned}
$$

So, in particular, $\mathcal{S}\left(\Phi_{j}\right) \subset \mathcal{S}\left(\Phi_{j+1}\right), \mathcal{S}\left(\tilde{\Phi}_{j}\right) \subset \mathcal{S}\left(\tilde{\Phi}_{j+1}\right)$ and $\mathcal{S}\left(\Upsilon_{j+1}\right)=\mathcal{S}\left(\Phi_{j+1}\right)$. 
5. Biorthogonal space decompositions and wavelets. We have constructed primal and dual sequences of nested spaces that satisfy Jackson and Bernstein estimates. To conclude existence and stability, with respect to a range of Sobolev norms, of the corresponding biorthogonal space decompositions, the only thing left to show is the existence and uniform $L_{2}(\Gamma)$-boundedness of the biorthogonal projector.

Results similar to the next lemma are often used in the context of saddle point problems. A proof of (the non-trivial part of) this lemma can be found in, e.g., [14, Theorem 2.1(a)].

Lemma 5.1. Let $V, U$ be closed subspaces of a Hilbert space $H$. Then the following statements are equivalent:

(a) $\gamma:=\inf _{0 \neq u \in U} \sup _{0 \neq v \in V} \frac{|\langle u, v\rangle|}{\|u\|\|v\|}>0$, and for any $v \in V$, there exists a $u \in U$ with $\langle u, v\rangle \neq 0$.

(b) There exists a bounded projector $Q: H \rightarrow H$ with $\Im(Q)=V$ and $\Im(I-Q)=U^{\perp}$, which is therefore appropriately called a biorthogonal projector.

In either case it holds that $\gamma=\|Q\|^{-1}$, and the adjoint $Q^{*}$ satisfies $\Im\left(Q^{*}\right)=U$ and $\Im\left(I-Q^{*}\right)=V^{\perp}$.

When $\Sigma$ and $\Delta$ are Riesz bases for $U$ and $V$, respectively, then (a) or (b) are equivalent to the existence of a bounded inverse of $\langle\Sigma, \Delta\rangle: \ell_{2}(\Delta) \rightarrow \ell_{2}(\Sigma)$. In that case it holds that

$$
\left\|\langle\Sigma, \Sigma\rangle^{-1}\right\|^{-\frac{1}{2}}\left\|\langle\Delta, \Delta\rangle^{-1}\right\|^{-\frac{1}{2}} \leq \frac{\|Q\|}{\left\|\langle\Sigma, \Delta\rangle^{-1}\right\|} \leq\|\Sigma\|\|\Delta\| .
$$

To be able to transfer results valid on the reference parameter domain to the manifold, in particular those concerning $L_{2}(\square)$ - or $L_{2}(\Gamma)$-angles between spaces, we will have to assume that the coarsest "mesh" is sufficiently fine in order to control the influence of the generally non-constant Jacobian determinants.

Proposition 5.2. For $j \geq j_{0}$ being large enough, there exists a uniformly bounded projector $Q_{j}: L_{2}(\Gamma) \rightarrow L_{2}(\Gamma)$ with $\Im\left(Q_{j}\right)=\mathcal{S}\left(\Phi_{j}\right)$ and $\Im\left(I-Q_{j}\right)=$ $\mathcal{S}\left(\tilde{\Phi}_{j}\right)^{\perp_{L_{2}(\Gamma)}}$.

Proof. Setting

$$
\Delta_{j, q}^{\square}=\operatorname{diag}\left(\left|\partial \kappa_{q}(x)\right|\right)_{x \in I_{j}^{\square}},
$$

by $(\mathcal{L})$ for both $\Phi_{j}^{\square}, \tilde{\Phi}_{j}^{\square}$, the smoothness of $z \mapsto\left|\partial \kappa_{q}(z)\right|$, and the uniform boundedness of $\left\|\phi_{j, x}^{\square}\right\|_{L_{2}(\square)},\left\|\tilde{\phi}_{j, x}^{\square}\right\|_{L_{2}(\square)}$, we have

$$
\left\|\left\langle\Phi_{j}^{\square}, \tilde{\Phi}_{j}^{\square}\right\rangle_{L_{2}(\square),\left|\partial \kappa_{q}\right|}-\left(\Delta_{j, q}^{\square}\right)^{\frac{1}{2}}\left\langle\Phi_{j}^{\square}, \tilde{\Phi}_{j}^{\square}\right\rangle_{L_{2}(\square)}\left(\Delta_{j, q}^{\square}\right)^{\frac{1}{2}}\right\| \lesssim 2^{-j} .
$$

By assumption (J1) and $\left|\partial \kappa_{q}\right| \gtrsim 1$, we have

$$
\Re\left(\left(\Delta_{j, q}^{\square}\right)^{\frac{1}{2}}\left\langle\Phi_{j}^{\square}, \tilde{\Phi}_{j}^{\square}\right\rangle_{L_{2}(\square)}\left(\Delta_{j, q}^{\square}\right)^{\frac{1}{2}}\right)=\left(\Delta_{j, q}^{\square}\right)^{\frac{1}{2}} \Re\left\langle\Phi_{j}^{\square}, \tilde{\Phi}_{j}^{\square}\right\rangle_{L_{2}(\square)}\left(\Delta_{j, q}^{\square}\right)^{\frac{1}{2}} \gtrsim I d,
$$

so that for $j \geq j_{0}$ large enough, $\Re\left\langle\Phi_{j}^{\square}, \tilde{\Phi}_{j}^{\square}\right\rangle_{L_{2}(\square),\left|\partial \kappa_{q}\right|} \gtrsim I d$. Similarly to (4.3), we find that

$$
\Re\left\langle\Phi_{j}, \tilde{\Phi}_{j}\right\rangle_{L_{2}(\Gamma)}=\sum_{q=1}^{M} \mathbf{E}_{j, q} \Re\left\langle\Phi_{j}^{\square}, \tilde{\Phi}_{j}^{\square}\right\rangle_{L_{2}(\square),\left|\partial \kappa_{q}\right|} \mathbf{E}_{j, q}^{T} \gtrsim \sum_{q=1}^{M} \mathbf{E}_{j, q} \mathbf{E}_{j, q}^{T}=I d .
$$


Since, for $j \geq j_{0}$, apparently $\left\langle\Phi_{j}, \tilde{\Phi}_{j}\right\rangle_{L_{2}(\Gamma)}$ is uniformly boundedly invertible, and $\Phi_{j}$ and $\tilde{\Phi}_{j}$ are uniform $L_{2}(\Gamma)$-Riesz systems, an application of Lemma 5.1 completes the the proof.

For $j \geq j_{0}$, the nesting $\mathcal{S}\left(\tilde{\Phi}_{j}\right) \subset \mathcal{S}\left(\tilde{\Phi}_{j+1}\right)$ gives $Q_{j}^{*}=Q_{j+1}^{*} Q_{j}^{*}$ or $Q_{j}=Q_{j} Q_{j+1}$, from which it follows that

$$
\Im\left(Q_{j+1}-Q_{j}\right)=\mathcal{S}\left(\Phi_{j+1}\right) \cap \mathcal{S}\left(\tilde{\Phi}_{j}\right)^{\perp_{L_{2}(\Gamma)}} .
$$

Analogously, $\mathcal{S}\left(\Phi_{j}\right) \subset \mathcal{S}\left(\Phi_{j+1}\right)$ implies that

$$
\Im\left(Q_{j+1}^{*}-Q_{j}^{*}\right)=\mathcal{S}\left(\tilde{\Phi}_{j+1}\right) \cap \mathcal{S}\left(\Phi_{j}\right)^{\perp_{L_{2}(\Gamma)}} .
$$

From the Jackson estimates and Bernstein inequalities at primal and dual side (4.4), (4.5), (4.6) and (4.7), and the existence and uniform $L_{2}(\Gamma)$-boundedness of the biorthogonal projectors $Q_{j}$ from Proposition 5.2, we have

TheOREM 5.3 (cf. e.g. [8], [14, Theorem 2.1]). With $Q_{j_{0}-1}:=0$, it holds that

$$
\left\|\sum_{j=j_{0}}^{\infty} w_{j}\right\|_{s}^{2} \lesssim \sum_{j=j_{0}}^{\infty} 4^{s j}\left\|w_{j}\right\|_{L_{2}(\Gamma)}^{2}, \quad\left(w_{j} \in \Im\left(Q_{j}-Q_{j-1}\right), s \in(-\tilde{d}, \gamma)\right),
$$

and

$$
\sum_{j=j_{0}}^{\infty} 4^{s j}\left\|\left(Q_{j}-Q_{j-1}\right) u\right\|_{L_{2}(\Gamma)}^{2} \lesssim\|u\|_{s}^{2}, \quad\left(u \in \mathcal{H}_{s}(\Gamma), s \in(-\tilde{\gamma}, d)\right) .
$$

For $s \in(-\min \{\tilde{\gamma}, \tilde{d}\}, \min \{\gamma, d\}),\left(w_{j}\right)_{j \geq j_{0}} \mapsto \sum_{j=j_{0}}^{\infty} w_{j}$ and $u \mapsto\left(\left(Q_{j}-Q_{j-1}\right) u\right)_{j \geq j_{0}}$, which mappings are bounded in the sense of (5.3) and (5.4), are each others inverse.

Analogous results are valid with $\left(Q_{j}\right)$ replaced by $\left(Q_{j}^{*}\right)$ and with interchanged roles of $(\gamma, d)$ and $(\tilde{\gamma}, \tilde{d})$.

Next, we construct a uniform $L_{2}(\Gamma)$-Riesz basis for $\Im\left(Q_{j+1}-Q_{j}\right)$, which elements are called wavelets.

Proposition 5.4.

(a) For $j \geq j_{0}$ being large enough, there exists a uniformly bounded projector $\bar{Q}_{j}$ : $L_{2}(\Gamma) \rightarrow L_{2}(\Gamma)$ with $\Im\left(\bar{Q}_{j}\right)=\mathcal{S}\left(\Theta_{j}\right)$ and $\Im\left(I d-\bar{Q}_{j}\right)=\mathcal{S}\left(\tilde{\Phi}_{j}\right)^{\perp_{L_{2}(\Gamma)}}$.

(b) This projector can be computed as $\bar{Q}_{j} u=\left\langle u, \tilde{\Phi}_{j}\right\rangle_{L_{2}(\Gamma)}\left\langle\Theta_{j}, \tilde{\Phi}_{j}\right\rangle_{L_{2}(\Gamma)}^{-1} \Theta_{j}$.

(c) The collection of wavelets

$$
\Psi_{j}:=\Xi_{j}-\left\langle\Xi_{j}, \tilde{\Phi}_{j}\right\rangle_{L_{2}(\Gamma)}\left\langle\Theta_{j}, \tilde{\Phi}_{j}\right\rangle_{L_{2}(\Gamma)}^{-1} \Theta_{j}
$$

is a uniform $L_{2}(\Gamma)$-Riesz basis for $\mathcal{S}\left(\Phi_{j+1}\right) \cap \mathcal{S}\left(\tilde{\Phi}_{j}\right)^{\perp_{L_{2}(\Gamma)}}$.

So by taking $j_{0}$ to be the maximum of the values from (a) and that of Proposition 5.2, for $s \in(-\min \{\tilde{\gamma}, \tilde{d}\}, \min \{\gamma, d\})$,

$$
\Phi_{j_{0}} \cup \cup_{j \geq j_{0}} 2^{-s j} \Psi_{j} \text { is a Riesz basis for } \mathcal{H}_{s}(\Gamma) \text {, }
$$

and thus, in view of (2.4), when in addition $|s|<\frac{3}{2},|s|<s_{\Gamma} \notin \mathbb{N}$ or $|s| \leq s_{\Gamma} \in \mathbb{N}$, it is a Riesz basis for $\mathcal{H}^{s}(\Gamma)$.

Proof. (a) Since $\Theta_{j}$ and $\tilde{\Phi}_{j}$ are uniform $L_{2}(\Gamma)$-Riesz bases, by Lemma 5.1 we have to show that, for $j \geq j_{0}$ being large enough, $\left\langle\Theta_{j}, \tilde{\Phi}_{j}\right\rangle_{L_{2}(\Gamma)}$ is uniformly boundedly invertible, which follows from (J2) similarly to the proof of Proposition 5.2. 
(b) Using that $\left\langle\Theta_{j}, \tilde{\Phi}_{j}\right\rangle_{L_{2}(\Gamma)}$ is uniformly boundedly invertible, one easily verifies that $\bar{Q}_{j}$, as given in (b), indeed has the properties listed in (a).

(c) Let $u \in \mathcal{S}\left(\Phi_{j+1}\right)$, then $u=\mathbf{c}_{j}^{T} \Theta_{j}+\mathbf{d}_{j}^{T} \Xi_{j}$ with $\|u\|_{L_{2}(\Gamma)} \bar{\sim}\left(\left\|\mathbf{c}_{j}\right\|^{2}+\left\|\mathbf{d}_{j}\right\|^{2}\right)^{\frac{1}{2}}$. If, in addition, $u \in \mathcal{S}\left(\tilde{\Phi}_{j}\right)^{\perp_{L_{2}(\Gamma)}}$, then $u=\left(I d-\bar{Q}_{j}\right) u=\left(I d-\bar{Q}_{j}\right) \mathbf{d}_{j}^{T} \Xi_{j}=\mathbf{d}_{j}^{T} \Psi_{j}$, and so with $\|u\|_{L_{2}(\Gamma)} \lesssim\left(1+\left\|\bar{Q}_{j}\right\|_{L_{2}(\Gamma) \rightarrow L_{2}(\Gamma)}\right)\left\|\mathbf{d}_{j}^{T} \Xi_{j}\right\|_{L_{2}(\Gamma)} \lesssim\left\|\mathbf{d}_{j}\right\| \lesssim\|u\|_{L_{2}(\Gamma)}$. Noting that $\Psi_{j} \subset \mathcal{S}\left(\Phi_{j+1}\right) \cap \mathcal{S}\left(\tilde{\Phi}_{j}\right)^{\perp_{L_{2}(\Gamma)}}$, we conclude that it is a uniform $L_{2}(\Gamma)$-Riesz basis for this space. The last statements are now consequences of Theorem 5.3.

Note that Proposition 4.1 at the dual side implies that $\Psi_{j}=\left(\psi_{j, x}\right)_{x \in J_{j}}$, yielded by (5.5), satisfies

$$
\begin{aligned}
& \left|\left\langle\psi_{j, x}, u\right\rangle_{L_{2}(\Gamma)}\right|=\left|\left\langle\psi_{j, x},\left(I d-\tilde{P}_{j}\right) u\right\rangle_{L_{2}(\Gamma)}\right| \\
& \quad \lesssim 2^{-\tilde{d} j} \sum_{q=1}^{M}\left|u \circ \kappa_{q}\right|_{H^{\tilde{d}}\left(B\left(\kappa_{q}^{-1}\left(\operatorname{supp} \psi_{j, x} \cap \Gamma_{q}\right) ;(\tilde{\vartheta}+3 \tilde{\varepsilon}) 2^{-j}\right) \cap \square\right)} \quad\left(u \in \mathcal{H}_{\tilde{d}}(\Gamma)\right),
\end{aligned}
$$

which property of the collections $\Psi_{j}$, with $\tilde{\vartheta}+3 \tilde{\varepsilon}$ replaced by an arbitrary but fixed $\tilde{\eta} \geq 0$ and the semi-norms $|\cdot|_{H^{\tilde{d}}(\ldots)}$ replaced by the norms $\|\cdot\|_{H^{\tilde{d}}(\ldots)}$, will be referred to as the uniform cancellation property of order $\tilde{d}$.

6. Stability of approximate wavelet bases. Similarly to (4.3), the definition of the collections $\Theta_{j}$ and $\tilde{\Phi}_{j}$ via (4.1) shows that

$$
\left\langle\Theta_{j}, \tilde{\Phi}_{j}\right\rangle_{L_{2}(\Gamma)}=\sum_{q=1}^{M} \mathbf{E}_{j, q}\left\langle\Theta_{j}^{\square}, \tilde{\Phi}_{j}^{\square}\right\rangle_{L_{2}(\square),\left|\partial \kappa_{q}\right|} \mathbf{E}_{j, q}^{T} .
$$

So if, for each $q, z \mapsto\left|\partial \kappa_{q}(z)\right|$ is a constant function, then (J2) shows that $\left\langle\Theta_{j}, \tilde{\Phi}_{j}\right\rangle_{L_{2}(\Gamma)}$ is diagonal, and the collection of wavelets $\Psi_{j}$ given in (5.5) is uniformly local. Unfortunately, only a restricted class of manifolds can be described as the union of patches that are the images of $\square$ under parametrizations that have constant Jacobians. In case not all Jacobians are constants, then, generally, $\left\langle\Theta_{j}, \tilde{\Phi}_{j}\right\rangle_{L_{2}(\Gamma)}$ is not diagonal and its inverse is densely populated, so that (5.5) yields wavelets $\Psi_{j}$ that have global supports.

A possibility to circumvent this problem, pursued in [12], is to carry out the whole wavelet construction outlined so far using the modified scalar product $\left\langle\langle,\rangle_{0}\right.$ instead of $\langle,\rangle_{L_{2}(\Gamma)}$. Indeed, $\left\langle\left\langle\Theta_{j}, \tilde{\Phi}_{j}\right\rangle_{0}=\sum_{q=1}^{M} \mathbf{E}_{j, q}\left\langle\Theta_{j}^{\square}, \tilde{\Phi}_{j}^{\square}\right\rangle_{L_{2}(\square)} \mathbf{E}_{j, q}^{T}=\sum_{q=1}^{M} \mathbf{E}_{j, q} \mathbf{E}_{j, q}^{T}=I d\right.$, and so uniformly local wavelets are obtained. What is more, by employing this scalar product, it is always possible to take the coarsest level $j_{0}=0$.

As was already recognized in [12], this approach, however, has two limitations: Firstly, the obtained wavelets will be orthogonal to the constant function with respect to $\left\langle\langle,\rangle_{0}\right.$. As a consequence, if the function

$$
J: \cup_{q=1}^{M} \Gamma_{q} \rightarrow \mathbb{R}: x \mapsto\left|\partial \kappa_{q^{\prime}}\left(\kappa_{q^{\prime}}^{-1}(x)\right)\right| \quad \text { when } x \in \Gamma_{q}^{\prime}
$$

has discontinuities, or more precisely, cannot be extended to a continuous function on $\Gamma$, then wavelets with supports that are not contained in one patch will generally not have a zero mean value with respect to the canonical Lebesgue measure on $\Gamma$, meaning that they have no cancellation property with respect to $\langle\cdot, \cdot\rangle_{L_{2}(\Gamma)}$. The application of wavelets we focus on is that for the solution of differential- or integral equations in variational form using the duality pairing with respect to $\langle\cdot, \cdot\rangle_{L_{2}(\Gamma)}$ (taking a different 
scalar product here yields other disadvantages, cf. [14, §1.2]). For obtaining nearly sparse representations of these operators in wavelet coordinates, and with that algorithms of optimal computational complexity, the wavelets should have a cancellation property of sufficiently high order (cf. $[10,22]$ or the surveys $[9,3]$ ), with respect thus to $\langle\cdot, \cdot\rangle_{L_{2}(\Gamma)}$. In a non-adaptive setting, under circumstances it might be possible that the fact that only wavelets along the, lower dimensional, patch interfaces do not have cancellation properties does not spoil optimal complexity. In an adaptive setting, however, such an argument cannot be applied.

The second limitation has to do with the interpretation, for $s<0$, of the statement that $\Phi_{j_{0}} \cup \cup_{j \geq j_{0}} 2^{-s j} \Psi_{j}$ is a Riesz basis for $\mathcal{H}_{s}(\Gamma)$, which is a consequence of Theorem 5.3. In case biorthogonality is realized with respect to $\langle\cdot, \cdot\rangle_{L_{2}(\Gamma)}$, then an expansion in terms of the basis $\Phi_{j_{0}} \cup \cup_{j \geq j_{0}} 2^{-s j} \Psi_{j}$ should be interpreted as an element of $\mathcal{H}_{s}(\Gamma)$, i.e., as a functional, using the embedding $L_{2}(\Gamma) \rightarrow \mathcal{H}_{s}(\Gamma): u \mapsto(v \mapsto$ $\left.\langle v, u\rangle_{L_{2}(\Gamma)}\right)$. Replacing $\langle\cdot, \cdot\rangle_{L_{2}(\Gamma)}$ by $\left\langle\langle\cdot, \cdot\rangle_{0}\right.$ means that also the embedding should be changed into $u \mapsto\left(v \mapsto\left\langle\langle v, u\rangle_{0}\right)\right.$. One can show (cf. [18, §4]) that if $J$ has discontinuities, then for $s \leq-\frac{1}{2}$ a set $\Phi_{j_{0}} \cup \cup_{j \geq j_{0}} 2^{-s j} \Psi_{j}$ which is a Riesz basis for $\mathcal{H}_{s}(\Gamma)$ using the embedding $u \mapsto\left(v \mapsto\left\langle\langle v, u\rangle_{0}\right)\right.$ cannot be a Riesz basis for $\mathcal{H}_{s}(\Gamma)$ using the embedding $u \mapsto\left(v \mapsto\langle v, u\rangle_{L_{2}(\Gamma)}\right)$. For $s>-\frac{1}{2}$, the property of being a Riesz basis for $\mathcal{H}_{s}(\Gamma)$ is the same for both embeddings. Again, since in applications duality pairing with respect to $\langle\cdot, \cdot\rangle_{L_{2}(\Gamma)}$ is used, in this paper, the property of a collection of functions to be a Riesz basis for $\mathcal{H}_{s}(\Gamma)$ for $s<0$ will always be interpreted with respect to the canonical embedding $u \mapsto\left(v \mapsto\langle v, u\rangle_{L_{2}(\Gamma)}\right)$.

In this paper, we propose another approach to solve the problem that generally $\left\langle\Theta_{j}, \tilde{\Phi}_{j}\right\rangle_{L_{2}(\Gamma)}^{-1}$ is densely populated, so that the wavelets yielded by (5.5) have global supports. As we will see, $\left\langle\Theta_{j}, \tilde{\Phi}_{j}\right\rangle_{L_{2}(\Gamma)}^{-1}$ can be well approximated by uniformly local matrices, so that close to the collections of the wavelets $\Psi_{j}$, there are collections of uniformly local functions of which suitable ones might be applied instead. In the following main theorem of this paper we derive general criteria under which such approximate wavelets satisfy the same conditions as $\Psi_{j}$ concerning both stability with respect to a range of Sobolev norms and the order of the cancellation property, where moreover, in contrast to $\Psi_{j}$, they are uniformly local.

THEOREM 6.1. If, for $j \geq j_{0}$,

(i) $\breve{\Psi}_{j}=\left(\breve{\psi}_{j, x}\right)_{x \in J_{j}} \subset \mathcal{S}\left(\Phi_{j+1}\right)$ is uniformly local,

(ii) $\breve{\Psi}_{j}$ has the uniform cancellation property of order $\tilde{d}\left(\right.$ w.r.t. $\left.\langle\cdot, \cdot\rangle_{L_{2}(\Gamma)}\right)$,

(iii) for some $\omega \in(0,1),\left\|\Psi_{j}-\breve{\Psi}_{j}\right\|_{L_{2}(\Gamma)} \lesssim \omega^{j}$,

then, possibly for a larger value of $j_{0}$, for $s \in(-\min \{\tilde{\gamma}, \tilde{d}\}, \min \{\gamma, d\})$,

$$
\Phi_{j_{0}} \cup \cup_{j \geq j_{0}} 2^{-s j} \breve{\Psi}_{j} \text { is a Riesz basis for } \mathcal{H}_{s}(\Gamma) \text {, }
$$

and thus, in view of (2.4), when in addition $|s|<\frac{3}{2},|s|<s_{\Gamma} \notin \mathbb{N}$ or $|s| \leq s_{\Gamma} \in \mathbb{N}$, it is a Riesz basis for $\mathcal{H}^{s}(\Gamma)$.

The rather lengthy proof of this theorem is postponed to Appendix A. The new aspect of this theorem is that instead of assuming (iii) with $\omega \leq 2^{-\min \{\tilde{\gamma}, \tilde{d}\}}$, which would yield the statement by "brute force" arguments, it is allowed that $\omega$ is arbitrarily close to 1 when, in addition, (ii) is valid, which property we like to have anyway. So although in two of our three constructions of approximate wavelets in the next section, $\omega$ will be equal to $\frac{1}{2}$, we nevertheless thus end up with Riesz bases for 
$\mathcal{H}_{s}(\Gamma)$ for the full range of $s$ allowed by $\tilde{\gamma}, \tilde{d}, \gamma$, and $d$. It is easily seen that (ii) alone is not sufficient to guarantee that the approximate wavelets generate a Riesz basis for any $\mathcal{H}_{s}(\Gamma)$.

The proof of Theorem 6.1 is based on perturbation arguments making use of the fact that we know that the true $L_{2}(\Gamma)$-biorthogonal wavelets generate Riesz bases for the full range of Sobolev spaces. We derived this fact in Section 5 by generalizing upon the well-known concept of stable completions developed in [2]. Note that in our setting we did not have explicit knowledge of $L_{2}(\Gamma)$-biorthogonal collections of scaling functions that are Riesz bases for $L_{2}(\Gamma)$. Theorem 6.1 and the applications in the following sections show the value of this generalization.

Remark 6.2. The approximate wavelets $\breve{\Psi}_{j}$ we are going to construct will be of type $\breve{\Psi}_{j}=\Xi_{j}-\mathbf{Z}_{j} \Theta_{j}$, where $\mathbf{Z}_{j}$ is a uniformly local $\# J_{j} \times \# I_{j}$ matrix. Since the basis transformation $\left\langle\Upsilon_{j+1}, \Lambda_{j+1}\right\rangle_{L_{2}(\Gamma)}^{T}$ from $\Upsilon_{j+1}=\left[\begin{array}{ll}\Theta_{j}^{T} & \Xi_{j}^{T}\end{array}\right]^{T}$ to $\Phi_{j+1}$ is uniformly local, so is the basis transformation from $\left[\begin{array}{cc}\Theta_{j}^{T} & \breve{\Psi}_{j}^{T}\end{array}\right]^{T}$ to $\Phi_{j+1}$, and the transformation from multi-scale basis $\Phi_{j_{0}} \cup \cup_{k=j_{0}}^{j-1} \breve{\Psi}_{k}$ to single-scale basis $\Phi_{j}$ has linear complexity.

In the special case that $\Theta_{j}=\Phi_{j}$ and $\left\langle\Upsilon_{j+1}, \Lambda_{j+1}\right\rangle_{L_{2}(\Gamma)}^{-T}$ is uniformly local, which by Proposition 4.3 holds assuming (3.2), the basis transformation from $\Phi_{j+1}$ to $\left[\begin{array}{cc}\Phi_{j}^{T} & \breve{\Psi}_{j}^{T}\end{array}\right]^{T}$ will also be uniformly local, and so the inverse transformation from single-scale basis $\Phi_{j}$ to multi-scale basis $\Phi_{j_{0}} \cup \cup_{k=j_{0}}^{j-1} \breve{\Psi}_{k}$ has also linear complexity. However, since $\mathcal{S}\left(\breve{\Psi}_{j}\right)$ is only approximately $L_{2}(\Gamma)$-orthogonal to $\mathcal{S}\left(\Phi_{j}\right)$, the corresponding dual wavelets will not be explicitly given.

Remark 6.3. Under the assumptions as in Remark 3.2, by using their uniform locality, one may verify that, for some constant $\tilde{\eta} \geq 0$ and $p \in[1, \infty]$, the approximate wavelets $\breve{\Psi}_{j}$ that will be constructed in the next section satisfy

$$
\left|\left\langle\breve{\psi}_{j, x}, u\right\rangle_{L_{2}(\Gamma)}\right| \lesssim 2^{-\left(\tilde{d}+\frac{n}{2}-\frac{p}{2}\right) j} \sum_{q=1}^{M}\left\|u \circ \kappa_{q}\right\|_{W_{p}^{\tilde{d}}\left(B\left(\kappa_{q}^{-1}\left(\operatorname{supp} \psi_{j, x} \cap \Gamma_{q}\right) ; \tilde{\eta} 2^{-j}\right) \cap \square\right)},
$$

for $u \in \mathcal{W}_{p, \tilde{d}}(\Gamma)$, which space is defined as $\mathcal{H}_{\tilde{d}}(\Gamma)$ with the $H^{\tilde{d}}(\square)$-norms replaced by $W_{p}^{\tilde{d}}(\square)$-norms. This generalization of the uniform cancellation property of order $\tilde{d}$ has some useful applications for proving results about matrix compression.

\section{Construction of uniformly local approximate wavelet bases.}

7.1. Approximating $\left\langle\Theta_{j}, \tilde{\Phi}_{j}\right\rangle_{L_{2}(\Gamma)}^{-1}$ using Jacobi iteration. As we will see, for $j \rightarrow \infty$, the matrix $\left\langle\Theta_{j}, \tilde{\Phi}_{j}\right\rangle_{L_{2}(\Gamma)}$ is increasingly close to its diagonal, and so it makes sense to approximate its inverse by a few Jacobi iteration steps ( $\tilde{d}$ steps will be sufficient). We will denote the resulting collection of approximate wavelets as as $\Psi_{j}^{\mathrm{Jc}}$, where "Jc" refers to Jacobi iteration.

Theorem 7.1. With $\mathbf{D}_{j}:=\operatorname{diag}\left\langle\Theta_{j}, \tilde{\Phi}_{j}\right\rangle_{L_{2}(\Gamma)}$, and for $j \geq j_{0}$ large enough,

$$
\Psi_{j}^{\mathrm{Jc}}:=\Xi_{j}-\left\langle\Xi_{j}, \tilde{\Phi}_{j}\right\rangle_{L_{2}(\Gamma)}\left[\sum_{k=0}^{\tilde{d}-1}\left(I d-\mathbf{D}_{j}^{-1}\left\langle\Theta_{j}, \tilde{\Phi}_{j}\right\rangle_{L_{2}(\Gamma)}\right)^{k} \mathbf{D}_{j}^{-1}\right] \Theta_{j}
$$

is uniformly local, it has the uniform cancellation property of order $\tilde{d}$, and finally, for $s \in(-\min \{\tilde{\gamma}, \tilde{d}\}, \min \{\gamma, d\})$,

$$
\Phi_{j_{0}} \cup \cup_{j \geq j_{0}} 2^{-s j} \Psi_{j}^{\mathrm{Jc}} \text { is a Riesz basis for } \mathcal{H}_{s}(\Gamma) \text {, }
$$


and thus, in view of (2.4), when in addition $|s|<\frac{3}{2},|s|<s_{\Gamma} \notin \mathbb{N}$ or $|s| \leq s_{\Gamma} \in \mathbb{N}$, it is a Riesz basis for $\mathcal{H}^{s}(\Gamma)$.

Proof. With $\Delta_{j, q}^{\square}$ as defined in (5.1), and by using (J2), similar as in (5.2) we have $\left\|\left\langle\Theta_{j}^{\square}, \tilde{\Phi}_{j}^{\square}\right\rangle_{L_{2}(\square),\left|\partial \kappa_{q}\right|}-\Delta_{j, q}^{\square}\right\| \lesssim 2^{-j}$. Since

$$
\overline{\mathbf{D}}_{j}:=\sum_{q=1}^{M} \mathbf{E}_{j, q} \Delta_{j, q}^{\square} \mathbf{E}_{j, q}^{T}
$$

is diagonal, by (6.1) we have

$$
\begin{aligned}
\left\|\mathbf{D}_{j}-\overline{\mathbf{D}}_{j}\right\| & \leq \max _{x, y \in I_{j}}\left|\left(\mathbf{D}_{j}-\overline{\mathbf{D}}_{j}\right)_{x, y}\right| \leq \max _{x, y \in I_{j}}\left|\left(\left\langle\Theta_{j}, \tilde{\Phi}_{j}\right\rangle_{L_{2}(\Gamma)}-\overline{\mathbf{D}}_{j}\right)_{x, y}\right| \\
& \leq\left\|\left\langle\Theta_{j}, \tilde{\Phi}_{j}\right\rangle_{L_{2}(\Gamma)}-\overline{\mathbf{D}}_{j}\right\| \lesssim 2^{-j}
\end{aligned}
$$

and so $\left\|\mathbf{D}_{j}-\left\langle\Theta_{j}, \tilde{\Phi}_{j}\right\rangle_{L_{2}(\Gamma)}\right\| \leq\left\|\mathbf{D}_{j}-\overline{\mathbf{D}}_{j}\right\|+\left\|\overline{\mathbf{D}}_{j}-\left\langle\Theta_{j}, \tilde{\Phi}_{j}\right\rangle_{L_{2}(\Gamma)}\right\| \lesssim 2^{-j}$.

As we have seen in the proof of Proposition 5.4, for $j \geq j_{0}$ large enough, the matrix $\left\langle\Theta_{j}, \tilde{\Phi}_{j}\right\rangle_{L_{2}(\Gamma)}$ is uniformly boundedly invertible, and thus, possible for a larger $j_{0}$, so is $\mathbf{D}_{j}$. We infer that

$$
\begin{aligned}
\|\left\langle\Theta_{j}, \tilde{\Phi}_{j}\right\rangle_{L_{2}(\Gamma)}^{-1}- & \sum_{k=0}^{\tilde{d}-1}\left(I d-\mathbf{D}_{j}^{-1}\left\langle\Theta_{j}, \tilde{\Phi}_{j}\right\rangle_{L_{2}(\Gamma)}\right)^{k} \mathbf{D}_{j}^{-1} \| \\
& =\left\|\left\langle\Theta_{j}, \tilde{\Phi}_{j}\right\rangle_{L_{2}(\Gamma)}^{-1}\left(\left(\mathbf{D}_{j}-\left\langle\Theta_{j}, \tilde{\Phi}_{j}\right\rangle_{L_{2}(\Gamma)}\right) \mathbf{D}_{j}^{-1}\right)^{\tilde{d}}\right\| \lesssim 2^{-\tilde{d} j}
\end{aligned}
$$

and thus by $\left\|\left\langle\Xi_{j}, \tilde{\Phi}_{j}\right\rangle_{L_{2}(\Gamma)}\right\| \lesssim 1$, that

$$
\left\|\Psi_{j}-\Psi_{j}^{\mathrm{Jc}}\right\|_{L_{2}(\Gamma)} \lesssim 2^{-\tilde{d j} j}\left\|\Theta_{j}\right\|_{L_{2}(\Gamma)} \lesssim 2^{-\tilde{d} j}
$$

Since furthermore $\Psi_{j}^{\mathrm{Jc}}$ is uniformly local, in view of Theorem 6.1 the only thing left to show is that $\Psi_{j}^{\mathrm{Jc}}$ has the uniform cancellation property of order $\tilde{d}$.

Although $\Psi_{j}$ has the uniform cancellation property of order $\tilde{d}$, we cannot immediately conclude this from (7.2) for $\Psi_{j}^{\mathrm{Jc}}$. Indeed, since the wavelets from $\Psi_{j}$ generally have global supports, invoking (7.2) and the cancellation property of $\psi_{j, x}$ would yield a bound for $\left|\left\langle\psi_{j, x}^{\mathrm{Jc}}, u\right\rangle_{L_{2}(\Gamma)}\right|$ in terms of the global $H^{d}$-norms of $u \circ \kappa_{q}$, whereas the definition of the cancellation property requires a bound in terms of the $H^{d}$-norms of $u \circ \kappa_{q}$ in a neighbourhood of $\operatorname{supp}\left(\psi_{j, x}^{\mathrm{Jc}} \circ \kappa_{q}\right)$ with diameter of order $2^{-j}$. To arrive at this result, we split $u$ into $\left(I d-\tilde{P}_{j}\right) u$ and $\tilde{P}_{j} u$, and then replace $\tilde{P}_{j} u$ by a function, equal to $\tilde{P}_{j} u$ on $\operatorname{supp} \psi_{j, x}^{\mathrm{Jc}}$ and still in $\mathcal{S}\left(\tilde{\Phi}_{j}\right)$, that has a support with diameter of order $2^{-j}$. The details are given below.

Using $\left\|\Psi_{j}^{\mathrm{Jc}}\right\|_{L_{2}(\Gamma)} \leq\left\|\Psi_{j}^{\mathrm{Jc}}-\Psi_{j}\right\|_{L_{2}(\Gamma)}+\left\|\Psi_{j}\right\|_{L_{2}(\Gamma)} \lesssim 1$, from the analogue of Proposition 4.1 at the dual side, for $u \in \mathcal{H}_{\tilde{d}}(\Gamma)$ we have

$$
\left|\left\langle\psi_{j, x}^{\mathrm{Jc}},\left(I d-\tilde{P}_{j}\right) u\right\rangle_{L_{2}(\Gamma)}\right| \lesssim 2^{-\tilde{d} j} \sum_{q=1}^{M}\left|u \circ \kappa_{q}\right|_{H^{\tilde{d}}\left(B\left(\kappa_{q}^{-1}\left(\operatorname{supp} \psi_{j, x}^{\mathrm{Jc}} \cap \Gamma_{q}\right) ;(\tilde{\vartheta}+3 \tilde{\varepsilon}) 2^{-j}\right) \cap \square\right)}
$$

We set

$$
I_{j}(x):=\left\{y \in I_{j}: \operatorname{supp} \tilde{\phi}_{j, y} \cap \operatorname{supp} \psi_{j, x}^{\mathrm{Jc}} \neq \emptyset\right\}, \text { and } \tilde{P}_{j, x}: u \mapsto \sum_{y \in I_{j}(x)} \tilde{\lambda}_{j, y}(u) \tilde{\phi}_{j, y} .
$$


Noting that $\psi_{j, x} \perp_{L_{2}(\Gamma)} \mathcal{S}\left(\tilde{\Phi}_{j}\right)$, and that $\# I_{j}(x)$ is bounded, uniformly in $j$ and $x \in J_{j}$, we have

$$
\begin{aligned}
& (7.5)\left|\left\langle\psi_{j, x}^{\mathrm{Jc}}, \tilde{P}_{j} u\right\rangle_{L_{2}(\Gamma)}\right| \\
& =\left|\left\langle\psi_{j, x}^{\mathrm{Jc}}, \tilde{P}_{j, x} u\right\rangle_{L_{2}(\Gamma)}\right|=\left|\left\langle\psi_{j, x}^{\mathrm{Jc}}-\psi_{j, x}, \tilde{P}_{j, x} u\right\rangle_{L_{2}(\Gamma)}\right| \\
& \lesssim 2^{-\tilde{d} j} \sum_{q=1}^{M}\left\|\sum_{z \in \kappa_{q}^{-1}\left(I_{j}(x)\right)} \tilde{\lambda}_{j, z}^{\square}\left(u \circ \kappa_{q}\right) \tilde{\phi}_{j, z}^{\square}\right\|_{L_{2}(\square)} \lesssim 2^{-\tilde{d} j} \max _{1 \leq q \leq M, z \in \kappa_{q}^{-1}\left(I_{j}(x)\right)}\left|\tilde{\lambda}_{j, z}^{\square}\left(u \circ \kappa_{q}\right)\right| .
\end{aligned}
$$

It is possible that for some $1 \leq q^{\prime} \leq M, \kappa_{q^{\prime}}^{-1}\left(I_{j}(x)\right) \neq \emptyset$, whereas $\operatorname{supp} \psi_{j, x}^{\mathrm{Jc}} \cap \Gamma_{q^{\prime}}=\emptyset$. Yet, for such $q^{\prime}$, any $z^{\prime} \in \kappa_{q^{\prime}}^{-1}\left(I_{j}(x)\right)$ is on a face of $\square$, and there exist $q$ and $z \in$ $\kappa_{q}^{-1}\left(I_{j}(x)\right)$ with $\operatorname{supp} \psi_{j, x}^{\mathrm{Jc}} \cap \Gamma_{q} \neq \emptyset$ and $\kappa_{q^{\prime}}\left(z^{\prime}\right)=\kappa_{q}(z)$, and thus $\tilde{\lambda}_{j, z^{\prime}}^{\square}\left(u \circ \kappa_{q^{\prime}}\right)=$ $\tilde{\lambda}_{j, z}^{\square}\left(u \circ \kappa_{q}\right)$, meaning that it suffices to bound $\left|\tilde{\lambda}_{j, z}^{\square}\left(u \circ \kappa_{q}\right)\right|$ for such $q$ and $z$. Since $\operatorname{dist}\left(z, \kappa_{q}^{-1}\left(\operatorname{supp} \psi_{j, x}^{\mathrm{Jc}} \cap \Gamma_{q}\right)\right) \leq \tilde{\varepsilon} 2^{-j}$, and $\left|\tilde{\lambda}_{j, z}^{\square}\left(u \circ \kappa_{q}\right)\right| \lesssim\left\|u \circ \kappa_{q}\right\|_{L_{2}\left(B\left(z, \tilde{\vartheta} 2^{-j}\right) \cap \square\right)}+$ $2^{-\tilde{d} j}\left|u \circ \kappa_{q}\right|_{H^{\tilde{d}}\left(B\left(z, \tilde{\vartheta} 2^{-j}\right) \cap \square\right)}$, as follows from (3.1) at the dual side, we conclude that

$$
\begin{aligned}
\left|\left\langle\psi_{j, x}^{\mathrm{Jc}}, \tilde{P}_{j} u\right\rangle_{L_{2}(\Gamma)}\right| \lesssim & 2^{-\tilde{d} j} \sum_{q=1}^{M}\left[\left\|u \circ \kappa_{q}\right\|_{L_{2}\left(B\left(\kappa_{q}^{-1}\left(\operatorname{supp} \psi_{j, x}^{\mathrm{Jc}} \cap \Gamma_{q}\right) ;(\tilde{\vartheta}+\tilde{\varepsilon}) 2^{-j}\right) \cap \square\right)}\right. \\
& \left.+2^{-\tilde{d} j}\left|u \circ \kappa_{q}\right|_{H^{\tilde{d}}\left(B\left(\kappa_{q}^{-1}\left(\operatorname{supp} \psi_{j, x}^{\mathrm{Jc}} \cap \Gamma_{q}\right) ;(\tilde{\vartheta}+\tilde{\varepsilon}) 2^{-j}\right) \cap \square\right)}\right] .
\end{aligned}
$$

The combination of (7.3) and (7.6) completes the proof.

Remark 7.2. The construction of the approximate wavelets $\Psi_{j}^{\mathrm{Jc}}$ in Theorem 7.1 has some similarities to the construction of approximate "prewavelets" in [23], where the inverse of a mass matrix with respect to a standard finite element basis is approximated by a number of steps of an iterative method, as the Jacobi or symmetric Gauss-Seidel method. A difference is that in our case the matrix $\mathbf{D}_{j}$ converges to $\left\langle\Theta_{j}, \tilde{\Phi}_{j}\right\rangle_{L_{2}(\Gamma)}$ as $j \rightarrow \infty$, allowing us to derive much stronger results concerning the generation of Riesz bases by the resulting approximate wavelets.

Compared to the approximate wavelets one gets by simply replacing $\langle\cdot, \cdot\rangle_{L_{2}(\Gamma)}$ by $\left\langle\langle\cdot, \cdot\rangle_{0}\right.$ in $(5.5)$, for $\tilde{d} \geq 2$ the approximate wavelets $\psi_{j, x}^{\mathrm{Jc}}$ have relatively large supports. Although this has not so much an effect on the multi-scale to single scale transform, that can be implemented being much more efficient than suggested by the sizes of the supports, it is a disadvantage for example when it concerns the compression of the stiffness matrix of an integral operator with respect to these approximate wavelets. In the following two subsections, we construct approximate wavelets with smaller supports.

As a preparation, the next proposition facilitates the verification of the third condition from Theorem 6.1, in case different constructions of approximate wavelets are used on different parts of $\Gamma$. In the proof, the problem of the generally global supports of the true biorthogonal wavelets is circumvented by approximating them by sufficiently accurate, uniformly local approximate wavelets generated by the Jacobi iteration approach.

Proposition 7.3. Let $\omega \in(0,1)$ and let $\breve{\Psi}_{j}=\left(\breve{\psi}_{j, x}\right)_{x \in J_{j}}$ be uniformly local. Then $\left\|\Psi_{j}-\breve{\Psi}_{j}\right\|_{L_{2}(\Gamma)} \lesssim \omega^{j}$ if and only if $\sup _{x \in J_{j}}\left\|\psi_{j, x}-\breve{\psi}_{j, x}\right\|_{L_{2}(\Gamma)} \lesssim \omega^{j}$.

Proof. Let $\sup _{x \in J_{j}}\left\|\psi_{j, x}-\breve{\psi}_{j, x}\right\|_{L_{2}(\Gamma)} \lesssim \omega^{j}$. Selecting $m \in \mathbb{N}$ such that $2^{-m} \leq \omega$, from the proof of Theorem 7.1 we learn that there exists a uniformly local $\Psi_{j}^{\mathrm{Jc}}$ with 
$\left\|\Psi_{j}-\Psi_{j}^{\mathrm{Jc}}\right\|_{L_{2}(\Gamma)} \lesssim 2^{-m j} \leq \omega^{j}$, and $\operatorname{so} \sup _{x \in J_{j}}\left\|\psi_{j, x}-\psi_{j, x}^{\mathrm{Jc}}\right\|_{L_{2}(\Gamma)} \lesssim \omega^{j}$ and thus $\sup _{x \in J_{j}}\left\|\psi_{j, x}^{\mathrm{Jc}}-\breve{\psi}_{j, x}\right\|_{L_{2}(\Gamma)} \lesssim \omega^{j}$. Since both $\Psi_{j}^{\mathrm{Jc}}$ and $\breve{\Psi}_{j}$ are uniformly local, this implies $\left\|\Psi_{j}^{\mathrm{Jc}}-\breve{\Psi}_{j}\right\|_{L_{2}(\Gamma)} \lesssim \omega^{j}$, and thus that $\left\|\Psi_{j}-\breve{\Psi}_{j}\right\|_{L_{2}(\Gamma)} \leq\left\|\Psi_{j}-\Psi_{j}^{\mathrm{Jc}}\right\|_{L_{2}(\Gamma)}+$ $\left\|\Psi_{j}^{\mathrm{Jc}}-\breve{\Psi}_{j}\right\|_{L_{2}(\Gamma)} \lesssim \omega^{j}$. The proof of the other implication is trivial.

7.2. Ignoring the Jacobian determinants away from the interfaces. In this subsection, we show that away from the patch interfaces, we may replace the wavelets from $\Psi_{j}^{\mathrm{Jc}}$ by the corresponding ones from

$$
\Psi_{j}^{(0)}:=\Xi_{j}-\left\langle\left\langle\Xi_{j}, \tilde{\Phi}_{j}\right\rangle_{0} \Theta_{j},\right.
$$

which is the collection of biorthogonal wavelets one obtains when biorthogonality is realized with respect to $\left\langle\langle,\rangle_{0}\right.$ instead of $\langle,\rangle_{L_{2}(\Gamma)}$, i.e., when, in the wavelet formula (5.5), all Jacobian determinants are replaced by the constant 1. and

Recalling that for $x \in \bar{\Gamma}, k(x)=\#\left\{q: x \in \overline{\Gamma_{q}}\right\}$, we set $I_{j}^{\circ}=\left\{x \in I_{j}: k(x)=1\right\}$

$$
J_{j}^{\circ}=\left\{x \in J_{j}: k(x)=1 \text { and }\left\langle\xi_{j, x}, \tilde{\phi}_{j, y}\right\rangle_{L_{2}(\Gamma)}=0 \text { for all } y \in I_{j} \backslash I_{j}^{\circ}\right\},
$$

which set is designed such that for $x \in J_{j}^{\circ}, \psi_{j, x}^{(0)}$ is fully supported inside one patch $\overline{\Gamma_{q}}$.

TheOREm 7.4. The set $\breve{\Psi}_{j}=\left\{\breve{\psi}_{j, x}: x \in J_{j}\right\}$, defined by $\breve{\psi}_{j, x}=\psi_{j, x}^{(0)}$ when $x \in J_{j}^{\circ}$, and $\breve{\psi}_{j, x}=\psi_{j, x}^{\mathrm{Jc}}$ when $x \in J_{j} \backslash J_{j}^{\circ}$, is uniformly local, it has the uniform cancellation property of order $\tilde{d}$, and for any $s \in(-\min \{\tilde{\gamma}, \tilde{d}\}, \min \{\gamma, d\})$ and $j_{0}$ large enough,

$$
\Phi_{j_{0}} \cup \cup_{j \geq j_{0}} 2^{-s j} \breve{\Psi}_{j} \text { is a Riesz basis for } \mathcal{H}_{s}(\Gamma)
$$

and thus, in view of (2.4), when in addition $|s|<\frac{3}{2},|s|<s_{\Gamma} \notin \mathbb{N}$ or $|s| \leq s_{\Gamma} \in \mathbb{N}$, it is a Riesz basis for $\mathcal{H}^{s}(\Gamma)$.

Proof. Using that, for $j \geq j_{0}$ large enough, $\left\|\left\langle\Theta_{j}, \tilde{\Phi}_{j}\right\rangle_{L_{2}(\Gamma)}^{-1}-\mathbf{D}_{j}^{-1}\right\| \lesssim 2^{-j}$, which follows as a special case from (7.1), and that the mappings $z \mapsto\left|\partial \kappa_{q}(z)\right|$ are smooth, one easily verifies that for $x \in J_{j}^{\circ}$,

$$
\left\|\psi_{j, x}-\psi_{j, x}^{(0)}\right\| \lesssim 2^{-j}
$$

Since for $x \in J_{j} \backslash J_{j}^{\circ},\left\|\psi_{j, x}-\psi_{j, x}^{\mathrm{Jc}}\right\| \lesssim 2^{-\tilde{d} j}$, and $\breve{\Psi}_{j}$ is uniformly local, in view of Theorem 6.1 and Proposition 7.3 the only thing left to show is that $\breve{\Psi}_{j}$ has the uniform cancellation property of order $\tilde{d}$. Knowing this for $\Psi_{j}^{\mathrm{Jc}}$, we only have to consider $\psi_{j, x}^{(0)}$ for $x \in J_{j}^{\circ}$.

Let $x \in J_{j}^{\circ}$, say $x \in \Gamma_{q}$, and let $u$ be a globally continuous, patchwise smooth function on $\Gamma$ that is zero on $\partial \Gamma_{D}$. Let $v$ be some arbitrary extension of the mapping $x \mapsto u(x)\left|\partial \kappa_{q}\left(\kappa_{q}^{-1}(x)\right)\right|$ on $\Gamma_{q}$ to a globally continuous, patchwise smooth function on $\Gamma$ that is zero on $\partial \Gamma_{D}$. Since $\operatorname{supp} \psi_{j, x}^{(0)} \subset \overline{\Gamma_{q}}$, from Proposition 4.1 at the dual side we have

$$
\begin{aligned}
\left|\left\langle\psi_{j, x}^{(0)}, u\right\rangle_{L_{2}(\Gamma)}\right| & =\left|\left\langle\left\langle\psi_{j, x}^{(0)}, v\right\rangle\right\rangle_{0}\right|=\left|\left\langle\left\langle\psi_{j, x}^{(0)},\left.\left(\left(I d-\tilde{P}_{j}\right) v\right)\right|_{\operatorname{supp} \psi_{j, x}^{(0)}}\right\rangle\right\rangle_{0}\right| \\
& \lesssim 2^{-\tilde{d} j}\left|v \circ \kappa_{q}\right|_{H^{\tilde{d}}\left(B\left(\kappa_{q}^{-1}\left(\operatorname{supp} \psi_{j, x}^{(0)}\right) ;(\tilde{\vartheta}+3 \tilde{\varepsilon}) 2^{-j}\right) \cap \square\right)} \\
& \lesssim 2^{-\tilde{d} j}\left\|u \circ \kappa_{q}\right\|_{H^{\tilde{d}}\left(B\left(\kappa_{q}^{-1}\left(\operatorname{supp} \psi_{j, x}^{(0)}\right) ;(\tilde{\vartheta}+3 \tilde{\varepsilon}) 2^{-j}\right) \cap \square\right)}
\end{aligned}
$$


which completes the proof.

Note that for $x \in J_{j}^{\circ}$, generally it only holds that $\left\|\psi_{j, x}-\psi_{j, x}^{(0)}\right\|_{L_{2}(\Gamma)} \approx 2^{-j}$. So in contrast to $\Psi_{j}^{\mathrm{Jc}}$, for the approximate wavelets $\breve{\Psi}_{j}$ from this subsection, generally $\left\|\Psi_{j}-\breve{\Psi}_{j}\right\|_{L_{2}(\Gamma)} \Varangle 2^{-\tilde{d} j}$ when $\tilde{d}>1$. The same will hold true for the collections $\breve{\Psi}_{j}$ that will be constructed in the next subsection. As follows from Theorem 6.1, however, this fact does not limit the range of $s$ for which the approximate wavelets generate a Riesz basis for $\mathcal{H}^{s}(\Gamma)$.

As we saw in the previous subsection, for $x \in J_{j}^{\circ}$ we can replace $\psi_{j, x}^{\mathrm{Jc}}$ by $\psi_{j, x}^{(0)}$, that, for $\tilde{d} \geq 2$, has a much smaller support. In this subsection, we investigate whether also near the interfaces we can find appropriate approximate wavelets $\psi_{j, x}$ with smaller supports.

We set

$$
\mathbb{P}_{\tilde{d}-2}(\Gamma):=C(\Gamma) \cap \prod_{q=1}^{M} \kappa_{q}\left(\mathbb{P}_{\tilde{d}-2}(\square)\right)
$$

where $\mathbb{P}_{\tilde{d}-2}(\square):=P_{\tilde{d}-2}(\square)$ when $\square$ is the interior of an $n$-simplex, and $\mathbb{P}_{\tilde{d}-2}(\square):=$ $Q_{\tilde{d}-2}(\square)$, being the tensor product space of the univariate polynomial spaces $P_{\tilde{d}-2}(0,1)$ in the $n$ coordinate directions, when $\square=(0,1)^{n}$. In the latter case, in addition to the assumption that $P_{\tilde{d}-1}(\square) \subset \mathcal{S}\left(\tilde{\Phi}_{j}^{\square}\right)((\mathcal{J})($ ii) at the dual side), in this subsection we assume that

$$
Q_{\tilde{d}-2}(\square) \subset \mathcal{S}\left(\tilde{\Phi}_{j}^{\square}\right)
$$

For $z \in \Gamma$ and $\varepsilon \geq 0$, let $B_{\Gamma}(z ; \varepsilon)=\left\{y \in \bar{\Gamma}: d_{\Gamma}(z, y) \leq \varepsilon\right\}$. With, for some constant $\rho \geq 0$, setting

$$
\tilde{V}_{j, x, \rho}=\left\{v \in \mathbb{P}_{\tilde{d}-2}(\Gamma):\left.v\right|_{\partial \Gamma_{D} \cap B_{\Gamma}\left(x ; \rho 2^{-j}\right)}=0\right\},
$$

for $x \in J_{j} \backslash J_{j}^{\circ}$ we will search

$$
\breve{\psi}_{j, x} \perp_{L_{2}(\Gamma)} \tilde{V}_{j, x, \rho} \text { with }\left\|\breve{\psi}_{j, x}-\hat{\psi}_{j, x}\right\|_{L_{2}(\Gamma)} \lesssim 2^{-j}
$$

We note that by taking $\tilde{V}_{j, x, \rho}$ to be the smaller set $\left\{v \in \mathbb{P}_{\tilde{d}-2}(\Gamma):\left.v\right|_{\partial \Gamma_{D}}=0\right\}, \breve{\psi}_{j, x}$ would not necessarily have the cancellation property of order $\tilde{d}$, and on the other hand, as we will see later, without incorporating boundary conditions in the definition of $\tilde{V}_{j, x, \rho}$, generally we cannot expect that $\left\|\breve{\psi}_{j, x}-\psi_{j, x}\right\|_{L_{2}(\Gamma)} \lesssim 2^{-j}$. For the moment assuming that such $\breve{\psi}_{j, x}$ can be found, which topic will be treated later in this subsection, the following Theorem 7.5 shows that they have the uniform cancellation property of order $\tilde{d}$, which, by Theorem 6.1, additionally yields the Riesz basis property for the full range of $s$. This may look surprising since, ignoring boundary conditions, the condition $\breve{\psi}_{j, x} \perp_{L_{2}(\Gamma)} \tilde{V}_{j, x, \rho}$ seems only to imply the uniform cancellation property of order $\tilde{d}-1$.

ThEOREM 7.5. Let $\breve{\Psi}_{j}=\left\{\breve{\psi}_{j, x}: x \in J_{j}\right\} \subset \mathcal{S}\left(\Phi_{j+1}\right)$ be a uniformly local set, with $\breve{\psi}_{j, x}=\psi_{j, x}^{(0)}$ when $x \in J_{j}^{\circ}$, and with for $x \in J_{j} \backslash J_{j}^{\circ},\left\|\breve{\psi}_{j, x}-\psi_{j, x}\right\|_{L_{2}(\Gamma)} \lesssim 2^{-j}$ and $\breve{\psi}_{j, x} \perp_{L_{2}(\Gamma)} \tilde{V}_{j, x, \rho}$. Then, for $j \geq j_{0}$ large enough, $\breve{\Psi}_{j}$ has the uniform cancellation property of order $\tilde{d}$, and for $s \in(-\min \{\tilde{\gamma}, \tilde{d}\}, \min \{\gamma, d\})$,

$$
\Phi_{j_{0}} \cup \cup_{j \geq j_{0}} 2^{-s j} \breve{\Psi}_{j} \text { is a Riesz basis for } \mathcal{H}_{s}(\Gamma) \text {, }
$$


and thus, in view of (2.4), when in addition $|s|<\frac{3}{2},|s|<s_{\Gamma} \notin \mathbb{N}$ or $|s| \leq s_{\Gamma} \in \mathbb{N}$, it is a Riesz basis for $\mathcal{H}^{s}(\Gamma)$.

Proof. In view of Theorem 6.1 and Proposition 7.3, it is sufficient to show that $\left\{\breve{\psi}_{j, x}: x \in J_{j} \backslash J_{j}^{\circ}\right\}$ has the uniform cancellation property of order $\tilde{d}$. We start by constructing, for given $u \in \mathcal{H}_{\tilde{d}}(\Gamma)$, a suitable interpolant in $\tilde{V}_{j, x, \rho}$. For $j \geq j_{0}$, and $x \in J_{j} \backslash J_{j}^{\circ}$, let $\mathcal{O}$ be the set of all $1 \leq q \leq M$ with $\operatorname{supp} \breve{\psi}_{j, x} \cap \Gamma_{q} \neq \emptyset$ or $B_{\Gamma}\left(x ; \rho 2^{-j}\right) \cap \Gamma_{q} \neq \emptyset$. For $j_{0}$ being large enough, for all $q \in \mathcal{O}$, we can find open, uniformly 'shape regular' open $n$-simplices or $n$ cubes $\square_{q}$ with $\operatorname{diam}\left(\square_{q}\right) \lesssim 2^{-j}$, and on each $\bar{\square}_{q}$, a set $K_{q}$ of $\operatorname{dim} \mathbb{P}_{\tilde{d}-2}(\square)$ interpolation points such that for given $u \in \mathcal{H}_{\tilde{d}}(\Gamma)$,

(a) $v \in \prod_{q \in \mathcal{O}} \kappa_{q}\left(\mathbb{P}_{\tilde{d}-2}(\square)\right)$ is uniquely determined by $\left(v \circ \kappa_{q}\right)\left(K_{q}\right)=\left(u \circ \kappa_{q}\right)\left(K_{q}\right)$ $(q \in \mathcal{O})$,

(b) $v \in C\left(\cup_{q \in \mathcal{O}} \overline{\Gamma_{q}}\right)$,

(c) for each $q \in \mathcal{O}$ and $z \in \overline{\square_{q}} \cap \kappa_{q}^{-1}\left(\partial \Gamma_{D}\right), v \circ \kappa_{q}$ vanishes on the lowest dimensional face $e$ of $\square$ that contains $z$.

For the last property, note that if $z \in \overline{\square_{q}} \cap \kappa_{q}^{-1}\left(\partial \Gamma_{D}\right)$, then by $(2.1), u \circ \kappa_{q}$ vanishes on the lowest dimensional face $e$ of $\square$, say of dimension $k$, that contains $z$. When $\square_{q}$ and $K_{q}$ are arranged such that $\square_{q}$ has a face $\tilde{e}$ of dimension $k$ on $e$, and $\left.\left(v \circ \kappa_{q}\right)\right|_{e}$ depends only on its values in $K_{q} \cap \tilde{e}$, indeed $v \circ \kappa_{q}$ vanishes on $e$. See Figure 7.1 for an illustration. The function $v$ can be extended to a function in $\mathbb{P}_{\tilde{d}-2}(\Gamma)$.

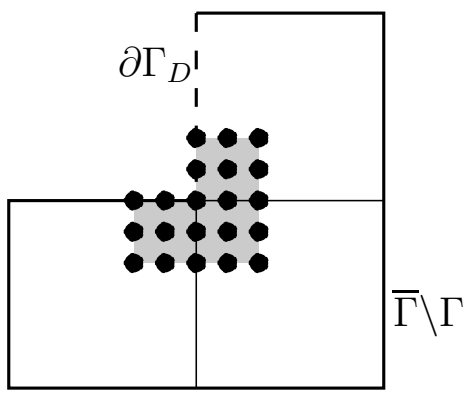

FIG. 7.1. Illustration with the proof of Theorem 7.5. $\cup_{q \in \mathcal{O}} \kappa_{q}\left(\square_{q}\right)$ and $\cup_{q \in \mathcal{O}} \kappa_{q}\left(K_{q}\right)$ for a $\Gamma$ consisting of 3 patches.

Since the above interpolation reproduces any $u \in C\left(\cup_{q \in \mathcal{O}} \overline{\Gamma_{q}}\right) \cap \prod_{q \in \mathcal{O}} \kappa_{q}\left(\mathbb{P}_{\tilde{d}-2}(\square)\right)$, standard arguments using the Sobolev embedding theorem (recall that $\tilde{d}>\frac{n}{2}$ ) and the Bramble-Hilbert lemma show that for $q \in \mathcal{O}$,

$$
\left\|(u-v) \circ \kappa_{q}\right\|_{L_{2}\left(\square_{q}\right)} \lesssim 2^{-(\tilde{d}-1) j}\left|u \circ \kappa_{q}\right|_{H^{\tilde{d}-1}\left(\square_{q}\right)}+2^{-\tilde{d} j}\left|u \circ \kappa_{q}\right|_{H^{\tilde{d}}\left(\square_{q}\right)} .
$$

Next we will select the above $\square_{q}$ to be sufficiently large such that $v \in \tilde{V}_{j, x, \rho}$ and $\left(I-\tilde{P}_{j}\right) v$ vanishes on $\operatorname{supp} \breve{\psi}_{j, x}$. Setting $\bar{I}_{j}=\cup_{q=1}^{M} \kappa_{q}\left(I_{j}^{\square}\right)$, i.e., without the exclusion of possible points on $\partial \Gamma_{D}$, and, for $y \in \bar{I}_{j} \backslash I_{j}$, defining $\tilde{\phi}_{j, y}$ and $\tilde{\lambda}_{j, y}$ similarly as in (4.1) and Proposition 4.1, respectively, we take the $\square_{q}$ to be sufficiently large such that

$$
\begin{aligned}
& B_{\Gamma}\left(x ; \rho 2^{-j}\right) \subset \cup_{q \in \mathcal{O}} \kappa_{q}\left(\overline{\square_{q}}\right), \\
&\left\{y \in \bar{I}_{j} \backslash I_{j}: \operatorname{supp} \tilde{\phi}_{j, y} \cap \operatorname{supp} \breve{\psi}_{j, x} \neq \emptyset\right\} \subset \cup_{q \in \mathcal{O}} \kappa_{q}\left(\overline{\square_{q}}\right), \\
& B\left(\kappa_{q}^{-1}\left(\operatorname{supp} \breve{\psi}_{j, x} \cap \Gamma_{q}\right) ;(\tilde{\vartheta}+\tilde{\varepsilon}) 2^{-j}\right) \cap \square \subset \square_{q} .
\end{aligned}
$$


From (c) and (7.9), we infer that $v \in \tilde{V}_{j, x, \rho}$, so that $\left\langle\breve{\psi}_{j, x}, v\right\rangle_{L_{2}(\Gamma)}=0$ by definition of $\breve{\psi}_{j, x}$. Because of the assumption ( $\tilde{\mathcal{J}}_{\mathrm{e}}$ ), the projector $w \mapsto \sum_{y \in \bar{I}_{j}}\left\langle w, \tilde{\lambda}_{j, y}\right\rangle_{L_{2}(\Gamma)} \tilde{\phi}_{j, y}$ reproduces any $v \in \mathbb{P}_{\tilde{d}-2}(\Gamma)$. By additionally using (7.10), (c), and (J)(ii), we have that $\left(I d-\tilde{P}_{j}\right) v$ vanishes on $\operatorname{supp} \breve{\psi}_{j, x}$. Writing

$$
\left\langle\breve{\psi}_{j, x}, u\right\rangle_{L_{2}(\Gamma)}=\left\langle\breve{\psi}_{j, x}, u-v\right\rangle_{L_{2}(\Gamma)}=\left\langle\breve{\psi}_{j, x},\left(I d-\tilde{P}_{j}\right) u\right\rangle_{L_{2}(\Gamma)}+\left\langle\breve{\psi}_{j, x}, \tilde{P}_{j}(u-v)\right\rangle_{L_{2}(\Gamma)},
$$

the first term can be estimated using Proposition 4.1 at the dual side.

From (7.4), recall the definition of $I_{j}(x)$ (with now $\psi_{j, x}^{\mathrm{Jc}}$ reading as $\breve{\psi}_{j, x}$ ) and that of the local projector $\tilde{P}_{j, x}$. Similar to (7.5) and (7.6) and using (7.11), for the second term we have

$$
\begin{aligned}
\left|\left\langle\breve{\psi}_{j, x}, \tilde{P}_{j}(u-v)\right\rangle_{L_{2}(\Gamma)}\right| & =\left|\left\langle\breve{\psi}_{j, x}, \tilde{P}_{j, x}(u-v)\right\rangle_{L_{2}(\Gamma)}\right|=\left|\left\langle\breve{\psi}_{j, x}-\psi_{j, x}, \tilde{P}_{j, x}(u-v)\right\rangle_{L_{2}(\Gamma)}\right| \\
& \lesssim 2^{-j} \sum_{q \in \mathcal{O}}\left[\left\|(u-v) \circ \kappa_{q}\right\|_{L_{2}\left(\square_{q}\right)}+2^{-\tilde{d} j}\left|u \circ \kappa_{q}\right|_{H^{\tilde{d}}\left(\square_{q}\right)}\right] \\
& \lesssim \sum_{q \in \mathcal{O}}\left[2^{-\tilde{d} j}\left|u \circ \kappa_{q}\right|_{H^{\tilde{d}-1}\left(\square_{q}\right)}+2^{-(\tilde{d}+1) j}\left|u \circ \kappa_{q}\right|_{H^{\tilde{d}\left(\square_{q}\right)}}\right]
\end{aligned}
$$

by (7.8), which yields the uniform cancellation property of order $\tilde{d}$, and so completes the proof. $\square$

Next, we discuss a construction of $\breve{\Psi}_{j}$ as in Theorem 7.5. Consider for $x \in J_{j} \backslash J_{j}^{\circ}$, the first order approximation $\hat{\psi}_{j, x}$ for $\psi_{j, x}$ from the collection

$$
\hat{\Psi}_{j}:=\Xi_{j}-\left\langle\Xi_{j}, \tilde{\Phi}_{j}\right\rangle_{L_{2}(\Gamma)} \mathbf{D}_{j}^{-1} \Theta_{j} .
$$

As a special case of $(7.1)$, we have $\left\|\psi_{j, x}-\hat{\psi}_{j, x}\right\|_{L_{2}(\Gamma)} \lesssim 2^{-j}$, where generally $\hat{\psi}_{j, x}$ only has the cancellation property of order 1 . We will construct $\breve{\psi}_{j, x}$ from $\hat{\psi}_{j, x}$ by adding correction terms. In view of our requirement that $\left\|\psi_{j, x}-\breve{\psi}_{j, x}\right\|_{L_{2}(\Gamma)} \lesssim 2^{-j}$, we first show that $\hat{\psi}_{j, x}$ is already nearly orthogonal to $\tilde{V}_{j, x, \rho}$, so that the correction can be small. For this to be true, the incorporation of boundary conditions in the definition of $\tilde{V}_{j, x, \rho}$ is essential.

LEMMA 7.6. Let the constant $\rho$ in the definition of $\tilde{V}_{j, x, \rho}$ be sufficiently large such that for all $x \in J_{j}$ and $y \in \bar{I}_{j}$ with $\operatorname{supp} \tilde{\phi}_{j, y} \cap \operatorname{supp} \hat{\psi}_{j, x} \neq \emptyset$, supp $\tilde{\lambda}_{j, y} \subset B_{\Gamma}\left(x ; \rho 2^{-j}\right)$. Then

$$
\left|\left\langle\hat{\psi}_{j, x}, p\right\rangle_{L_{2}(\Gamma)}\right| \lesssim 2^{-j}\|p\|_{L_{2}\left(\operatorname{supp} \hat{\psi}_{j, x}\right)} \quad\left(p \in \tilde{V}_{j, x, \rho}\right)
$$

Proof. Since $p \in \mathbb{P}_{\tilde{d}-2}(\Gamma)$, by the inclusion of possible points on $\partial \Gamma_{D}$ and $\left(\tilde{f}_{\mathrm{e}}\right)$, we have $p=\sum_{y \in \bar{I}_{j}}\left\langle p, \tilde{\lambda}_{j, y}\right\rangle_{L_{2}(\Gamma)} \tilde{\phi}_{j, y}$. Terms in this sum for $y \in \bar{I}_{j} \backslash I_{j}$ vanish on $\operatorname{supp} \hat{\psi}_{j, x}$ by (J)(ii) and because $p$ vanishes on $\partial \Gamma_{D} \cap B_{\Gamma}\left(x ; \rho 2^{-j}\right)$. Setting $p_{j, x}=$ $\sum_{\left\{y \in I_{j}: \operatorname{supp} \tilde{\phi}_{j, y} \cap \operatorname{supp} \hat{\psi}_{j, x} \neq \emptyset\right\}}\left\langle p, \tilde{\lambda}_{j, y}\right\rangle_{L_{2}(\Gamma)} \tilde{\phi}_{j, y}$, which is a function in $\mathcal{S}\left(\tilde{\Phi}_{j}\right)$, we find that

$$
\begin{aligned}
\left|\left\langle\hat{\psi}_{j, x}, p\right\rangle_{L_{2}(\Gamma)}\right|=\left|\left\langle\hat{\psi}_{j, x}, p_{j, x}\right\rangle_{L_{2}(\Gamma)}\right| & =\left|\left\langle\hat{\psi}_{j, x}-\psi_{j, x}, p_{j, x}\right\rangle_{L_{2}(\Gamma)}\right| \\
& \lesssim 2^{-j}\left\|p_{j, x}\right\|_{L_{2}(\Gamma)} \lesssim 2^{-j}\|p\|_{L_{2}\left(\operatorname{supp} \hat{\psi}_{j, x}\right)},
\end{aligned}
$$

where in the last step we used (J)(iv). 
Let us first consider the special case $\tilde{d}=2$ making the natural assumption that $\left|\int \xi_{j, x} d \mu\right| \gtrsim 2^{-j n / 2}$. Let $\rho$ be as in Lemma 7.6. If $x \in J_{j} \backslash J_{j}^{\circ}$ is such that $\partial \Gamma_{D} \cap$ $B_{\Gamma}\left(x ; \rho 2^{-j}\right) \neq \emptyset$, then $\tilde{V}_{j, x, \rho}=\{0\}$, and we can take $\breve{\psi}_{j, x}=\hat{\psi}_{j, x}$. Otherwise, we take $\breve{\psi}_{j, x}:=\hat{\psi}_{j, x}-\left[\int_{\Gamma} \hat{\psi}_{j, x} d \mu / \int_{\Gamma} \xi_{j, x} d \mu\right] \xi_{j, x}$. Obviously $\breve{\psi}_{j, x} \perp_{L_{2}(\Gamma)}$ 1, i.e., $\breve{\psi}_{j, x} \perp$ $\tilde{V}_{j, x, \rho}$, and Lemma 7.6 shows that $\left|\int_{\Gamma} \hat{\psi}_{j, x} d \mu\right| \lesssim 2^{-j(1+n / 2)}$, so that indeed $\| \hat{\psi}_{j, x}-$ $\breve{\psi}_{j, x} \|_{L_{2}(\Gamma)} \lesssim 2^{-j}$. In view of our aim to replace $\psi_{j, x}^{\mathrm{Jc}}$ for $x \in J_{j} \backslash J_{j}^{\circ}$ by an approximate wavelet with smaller support, note that the support of $\breve{\psi}_{j, x}$ is equal to that of $\hat{\psi}_{j, x}$ (which is equal to that of $\psi_{j, x}^{(0)}$ ).

For $\tilde{d}>2$, generally we have to add more than one degree of freedom to find a correction of $\hat{\psi}_{j, x}$ that is orthogonal to $\tilde{V}_{j, x, \rho}$. We will search the correction from the span of $\theta_{j, y}$ with $d_{\Gamma}(x, y) \lesssim 2^{-j}$. Instead of adding as many degrees of freedom as $\operatorname{dim}\left(\tilde{V}_{j, x, \rho}\right)$, generally we add more degrees of freedom, but then solve the resulting underdetermined problem in a minimal norm sense to end up with a correction term that is as small as possible. The resulting approximate wavelets will be denoted as $\psi_{j, x}^{\mathrm{ls}}$, where 'ls' refers to least-squares. In Theorem 7.7 it is stated that if, for sufficiently large $\delta$, we use all $\theta_{j, y}$ for $y \in I_{j}$ with $d_{\Gamma}(x, y) \leq \delta 2^{-j}$, then the constrained minimization problem has a unique solution $\psi_{j, x}^{\mathrm{ls}}$, with $\left\|\psi_{j, x}^{\mathrm{ls}}-\psi_{j, x}\right\|_{L_{2}(\Gamma)} \lesssim 2^{-j}$. Note that although in our numerical example we end up with $\psi_{j, x}^{\text {ls }}$ that has the same support as $\hat{\psi}_{j, x}$, which thus in particular is much smaller than the support of $\psi_{j, x}^{\mathrm{Jc}}$, we cannot prove this in general.

THEOREM 7.7. Let $\rho$ be as in Lemma 7.6. For a sufficiently large constant $\delta>0$, and with $\Theta_{j, x}^{\delta}:=\left\{\theta_{j, y}: y \in I_{j} \cap B_{\Gamma}\left(x ; \delta 2^{-j}\right)\right\}$, for any $x \in J_{j} \backslash J_{j}^{\circ}$ the problem of determining

$$
\underset{\psi_{j, x}^{\mathrm{ls}} \in \hat{\psi}_{j, x}+\mathcal{S}\left(\Theta_{j, x}^{\delta}\right)}{\operatorname{argmin}}\left\{\left\|\psi_{j, x}^{\mathrm{ls}}-\hat{\psi}_{j, x}\right\|_{L_{2}(\Gamma)}: \psi_{j, x}^{\mathrm{ls}} \perp_{L_{2}(\Gamma)} \tilde{V}_{j, x, \rho}\right\}
$$

has a unique solution with $\left\|\psi_{j, x}^{\mathrm{ls}}-\psi_{j, x}\right\|_{L_{2}(\Gamma)} \lesssim 2^{-j}$, so that Theorem 7.5 applies.

Proof. In the following, let $\eta>0$ be a constant such that

$$
\operatorname{supp} \tilde{\phi}_{j, y}, \operatorname{supp} \theta_{j, y} \subset B_{\Gamma}\left(y ; \eta 2^{-j}\right) \quad\left(y \in I_{j}\right)
$$

and let $\delta>0$ be a constant that will be fixed later, that, in any case, is sufficiently large such that

$$
\operatorname{supp} \hat{\psi}_{j, x} \subset B_{j, x}^{\delta}:=B_{\Gamma}\left(x ;(\delta+\eta) 2^{-j}\right) \quad\left(x \in J_{j}\right) .
$$

For $p \in \mathbb{P}_{\tilde{d}-2}(\Gamma)$, it holds that

$$
p-\sum_{y \in I_{j} \cap B_{\Gamma}\left(x ; \delta 2^{-j}\right)}\left\langle p, \tilde{\lambda}_{j, y}\right\rangle_{L_{2}(\Gamma)} \tilde{\phi}_{j, y}=\sum_{y \in \bar{I}_{j} \backslash\left(I_{j} \cap B_{\Gamma}\left(x ; \delta 2^{-j}\right)\right)}\left\langle p, \tilde{\lambda}_{j, y}\right\rangle_{L_{2}(\Gamma)} \tilde{\phi}_{j, y},
$$

and so, by the properties of the $\tilde{\phi}_{j, y}$ and $\tilde{\lambda}_{j, y}$, for some constant $\zeta>0$

$$
\begin{aligned}
& \left.\left\|p-\sum_{y \in I_{j} \cap B_{\Gamma}\left(x ; \delta 2^{-j}\right)}\left\langle p, \tilde{\lambda}_{j, y}\right\rangle_{L_{2}(\Gamma)} \tilde{\phi}_{j, y}\right\|_{L_{2}\left(B_{j, x}^{\delta}\right)} \sum_{\left\{y \in \bar{I}_{j} \backslash\left(I_{j} \cap B_{\Gamma}\left(x ; \delta 2^{-j}\right)\right): \operatorname{supp} \tilde{\phi}_{j, y} \cap B_{j, x}^{\delta} \neq \emptyset\right\}}\left|\left\langle p, \tilde{\lambda}_{j, y}\right\rangle_{L_{2}(\Gamma)}\right|^{2}\right]^{\frac{1}{2}} \\
& \lesssim\left[2^{-j n / 2}\|p\|_{L_{\infty}\left(B_{\Gamma}\left(x ;(\delta+\eta+\zeta) 2^{-j}\right)\right)}\left[\#\left\{y \in \bar{I}_{j} \backslash\left(I_{j} \cap B_{\Gamma}\left(x ; \delta 2^{-j}\right)\right): \operatorname{supp} \tilde{\phi}_{j, y} \cap B_{j, x}^{\delta} \neq \emptyset\right\}\right]^{\frac{1}{2}}\right. \\
& \lesssim \delta^{-n / 2}\|p\|_{L_{2}\left(B_{j, x}^{\delta}\right)} \times \delta^{(n-1) / 2},
\end{aligned}
$$


where we used that $p$ is a function from a fixed, finite dimensional space. See Figure 7.2 for an illustration. With $\tilde{\Phi}_{j, x}^{\delta}:=\left\{\tilde{\phi}_{j, y}: y \in I_{j} \cap B_{\Gamma}\left(x, \delta 2^{-j}\right)\right\}$, we conclude that

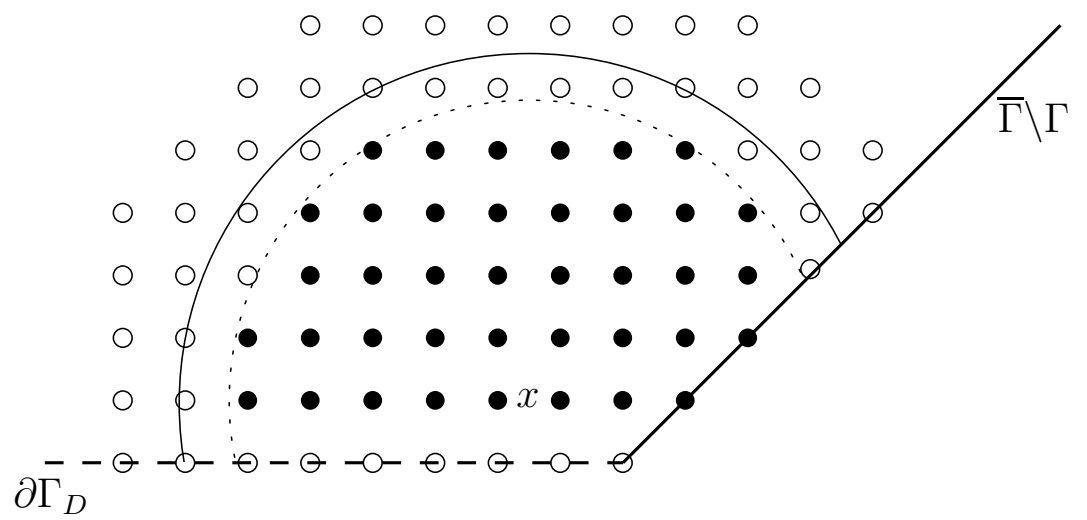

FIG. 7.2. Illustration with the proof of Theorem 7.7. $B_{j, x}^{\delta}\left(\right.$ solid circle), $I_{j} \cap B_{\Gamma}\left(x ; \delta 2^{-j}\right)(\bullet)$, $\bar{I}_{j} \backslash\left(I_{j} \cap B_{\Gamma}\left(x ; \delta 2^{-j}\right)\right)(\circ)$.

$$
\inf _{\tilde{v}_{j} \in \mathcal{S}\left(\tilde{\Phi}_{j, x}^{\delta}\right)}\left\|p-\tilde{v}_{j}\right\|_{L_{2}\left(B_{j, x}^{\delta}\right)} \lesssim \delta^{-\frac{1}{2}}\|p\|_{L_{2}\left(B_{j, x}^{\delta}\right)} \quad\left(p \in \mathbb{P}_{\tilde{d}-2}(\Gamma)\right)
$$

As we have seen, for $j \geq j_{0}$ large enough, the matrix $\left\langle\Theta_{j}, \tilde{\Phi}_{j}\right\rangle_{L_{2}(\Gamma)}$ is uniformly bounded invertible. Since furthermore $\left\|\left\langle\Theta_{j}, \tilde{\Phi}_{j}\right\rangle_{L_{2}(\Gamma)}-\operatorname{diag}\left\langle\Theta_{j}, \tilde{\Phi}_{j}\right\rangle_{L_{2}(\Gamma)}\right\| \lesssim 2^{-j}$, we infer that, for $j \geq j_{0}$ large enough, any principal submatrix of $\left\langle\Theta_{j}, \tilde{\Phi}_{j}\right\rangle_{L_{2}(\Gamma)}$ is uniformly boundedly invertible, again also with respect to the selection of this submatrix.

By using in addition that by (7.13) for any $y \in I_{j} \cap B_{\Gamma}\left(x, \delta 2^{-j}\right), \tilde{\phi}_{j, y}$ and $\theta_{j, y}$ vanish outside $B_{j, x}^{\delta}$, we infer that $\tilde{\Phi}_{j, x}^{\delta}$ and $\Theta_{j, x}^{\delta}$ are uniform $L_{2}\left(B_{j, x}^{\delta}\right)$-Riesz systems, with $\left\langle\tilde{\Phi}_{j, x}^{\delta}, \Theta_{j, x}^{\delta}\right\rangle_{L_{2}\left(B_{j, x}^{\delta}\right)}$ being uniformly boundedly invertible, where here as well as in the following "uniform" not only refers to $j \geq j_{0}$, but also to $x$ and $\delta$, as long as the latter has not been fixed.

By Lemma 5.1 there exists a uniformly bounded projector $\hat{Q}_{j, x}^{\delta}: L_{2}\left(B_{j, x}^{\delta}\right) \rightarrow$ $L_{2}\left(B_{j, x}^{\delta}\right)$ with $\Im\left(\hat{Q}_{j, x}^{\delta}\right)=\mathcal{S}\left(\tilde{\Phi}_{j, x}^{\delta}\right)$ and $\Im\left(I-\hat{Q}_{j, x}^{\delta}\right)=\mathcal{S}\left(\Theta_{j, x}^{\delta}\right)^{\perp_{L_{2}\left(B_{j, x}^{\delta}\right)} \text {. From }}$

$$
\left\|p-\hat{Q}_{j, x}^{\delta} p\right\|_{L_{2}\left(B_{j, x}^{\delta}\right)} \leq\left(1+\left\|\hat{Q}_{j, x}^{\delta}\right\|_{L_{2}\left(B_{j, x}^{\delta}\right) \rightarrow L_{2}\left(B_{j, x}^{\delta}\right)}\right) \inf _{\tilde{v}_{j} \in \mathcal{S}\left(\tilde{\Phi}_{j, x}^{\delta}\right)}\left\|p-\tilde{v}_{j}\right\|_{L_{2}\left(B_{j, x}^{\delta}\right)}
$$

for $p \in \mathbb{P}_{\tilde{d}-2}(\Gamma)$, we conclude that by fixing $\delta$ to be a sufficiently large constant, we have

$$
\left\|\hat{Q}_{j, x}^{\delta} p\right\|_{L_{2}\left(B_{j, x}^{\delta}\right)} \geq\|p\|_{L_{2}\left(B_{j, x}^{\delta}\right)}-\left\|p-\hat{Q}_{j, x}^{\delta} p\right\|_{L_{2}\left(B_{j, x}^{\delta}\right)} \gtrsim\|p\|_{L_{2}\left(B_{j, x}^{\delta}\right)},
$$

and thus

$$
\left\langle\left(\hat{Q}_{j, x}^{\delta}\right)^{*} \hat{Q}_{j, x}^{\delta} p, p\right\rangle_{\left.L_{2}\left(B_{j, x}^{\delta}\right)\right)} \gtrsim\|p\|_{L_{2}\left(B_{j, x}^{\delta}\right)}^{2} \gtrsim\left\|\left(\hat{Q}_{j, x}^{\delta}\right)^{*} \hat{Q}_{j, x}^{\delta} p\right\|_{L_{2}\left(B_{j, x}^{\delta}\right)}\|p\|_{L_{2}\left(B_{j, x}^{\delta}\right)} .
$$

Since this result is in particular valid for any $p \in \tilde{V}_{j, x, \rho}$, and $\Im\left(\left(\hat{Q}_{j, x}^{\delta}\right)^{*} \hat{Q}_{j, x}^{\delta}\right) \subset \mathcal{S}\left(\Theta_{j, x}^{\delta}\right)$, we have that

$$
\inf _{0 \neq p \in \tilde{V}_{j, x, \rho}} \sup _{0 \neq v_{j} \in \mathcal{S}\left(\Theta_{j, x}^{\delta}\right)} \frac{\left|\left\langle v_{j}, p\right\rangle_{L_{2}\left(B_{j, x}^{\delta}\right)}\right|}{\left\|v_{j}\right\|_{L_{2}\left(B_{j, x}^{\delta}\right)}\|p\|_{L_{2}\left(B_{j, x}^{\delta}\right)}} \gtrsim 1 .
$$


It is well-known that this so-called inf-sup condition implies that for any $u \in L_{2}\left(B_{j, x}^{\delta}\right)$, the problem of finding $v_{j} \in \mathcal{S}\left(\Theta_{j, x}^{\delta}\right)$ with minimal $L_{2}\left(B_{j, x}^{\delta}\right)$-norm satisfying

$$
\left\langle v_{j}, p\right\rangle_{L_{2}\left(B_{j, x}^{\delta}\right)}=\langle u, p\rangle_{L_{2}\left(B_{j, x}^{\delta}\right)} \quad\left(p \in \tilde{V}_{j, x, \rho}\right),
$$

has a unique solution, denoted as $v_{j}=L_{j, x}^{\delta} u$, for which $\left\|v_{j}\right\|_{L_{2}\left(B_{j, x}^{\delta}\right)} \lesssim\|u\|_{L_{2}\left(B_{j, x}^{\delta}\right)}$. Obviously, $L_{j, x}^{\delta}: L_{2}\left(B_{j, x}^{\delta}\right) \rightarrow \mathcal{S}\left(\Theta_{j, x}^{\delta}\right)$ is linear with $\operatorname{ker}\left(L_{j, x}^{\delta}\right)=\left(\left.\tilde{V}_{j, x, \rho}\right|_{B_{j, x}^{\delta}}\right)^{\perp_{L_{2}\left(B_{j, x}^{\delta}\right)}}$. Since supp $v_{j} \subset B_{j, x}^{\delta}$ for any $v_{j} \in \mathcal{S}\left(\Theta_{j, x}^{\delta}\right)$, for $u$ being supported in $B_{j, x}^{\delta}$ both $L_{2}\left(B_{j, x}^{\delta}\right)$ scalar products in (7.16) read as $L_{2}(\Gamma)$-scalar products. From supp $\hat{\psi}_{j, x} \subset B_{j, x}^{\delta}$ we conclude that the constrained minimization problem from the theorem has the unique solution $\psi_{j, x}^{\mathrm{ls}}=\left(I-L_{j, x}^{\delta}\right) \hat{\psi}_{j, x}$.

What is left to show is that $\left\|\psi_{j, x}^{\mathrm{ls}}-\hat{\psi}_{j, x}\right\|_{L_{2}(\Gamma)} \lesssim 2^{-j}$. Since supp $\hat{\psi}_{j, x} \subset B_{j, x}^{\delta}$, Lemma 7.6 shows that

$$
\left|\left\langle\hat{\psi}_{j, x}, p\right\rangle_{L_{2}\left(B_{j, x}^{\delta}\right)}\right|=\left|\left\langle\hat{\psi}_{j, x}, p\right\rangle_{L_{2}(\Gamma)}\right| \lesssim 2^{-j}\|p\|_{L_{2}\left(B_{j, x}^{\delta}\right)}, \quad\left(p \in \tilde{V}_{j, x, \rho}\right) .
$$

Since $\Im\left(\left(L_{j, x}^{\delta}\right)^{*}\right)=\operatorname{ker}\left(L_{j, x}^{\delta}\right)^{\perp_{L_{2}\left(B_{j, x}^{\delta}\right)}}=\left.\tilde{V}_{j, x, \rho}\right|_{B_{j, x}^{\delta}}$, we have

$$
\begin{aligned}
\left\|L_{j, x}^{\delta} \hat{\psi}_{j, x}\right\|_{L_{2}\left(B_{j, x}^{\delta}\right)} & =\sup _{0 \neq v \in L_{2}\left(B_{j, x}^{\delta}\right)} \frac{\left|\left\langle L_{j, x}^{\delta} \hat{\psi}_{j, x}, v\right\rangle_{L_{2}\left(B_{j, x}^{\delta}\right)}\right|}{\|v\|_{L_{2}\left(B_{j, x}^{\delta}\right)}} \\
& =\sup _{0 \neq v \in L_{2}\left(B_{j, x}^{\delta}\right)} \frac{\left|\left\langle\hat{\psi}_{j, x},\left(L_{j, x}^{\delta}\right)^{*} v\right\rangle_{L_{2}\left(B_{j, x}^{\delta}\right)}\right|}{\|v\|_{L_{2}\left(B_{j, x}^{\delta}\right)}} \\
& \lesssim 2^{-j}\left\|\hat{\psi}_{j, x}\right\|_{L_{2}\left(B_{j, x}^{\delta}\right)}\left\|\left(L_{j, x}^{\delta}\right)^{*}\right\|_{L_{2}\left(B_{j, x}^{\delta}\right) \rightarrow L_{2}\left(B_{j, x}^{\delta}\right)} \lesssim 2^{-j}\left\|\hat{\psi}_{j, x}\right\|_{L_{2}\left(B_{j, x}^{\delta}\right)},
\end{aligned}
$$

that is, $\left\|\psi_{j, x}^{\mathrm{ls}}-\hat{\psi}_{j, x}\right\|_{L_{2}(\Gamma)} \lesssim 2^{-j}$. [

Remark 7.8. In case $\Theta_{j} \neq \Phi_{j}$, it is not of any interest that $\breve{\Psi}_{j}-\Xi_{j} \in \mathcal{S}\left(\Theta_{j}\right)$ (cf. Remark 6.2). In that case, in Theorem 7.7 we may search $\psi_{j, x}^{\text {ls }}$ in the larger space $\hat{\psi}_{j, x}+\mathcal{S}\left(\Phi_{j+1, x}^{\delta}\right)$, with $\Phi_{j+1, x}^{\delta}:=\left\{\phi_{j+1, y}: x \in I_{j+1} \cap B_{\Gamma}\left(x ; \delta 2^{-j}\right)\right\}$, which opens the possibility that we may take a smaller $\delta$, and so reduce the support of the resulting $\psi_{j, x}^{\mathrm{ls}}$.

Remark 7.9. Both the construction of $\psi_{j, x}^{\mathrm{Jc}}$ from Theorem 7.1, and that of $\psi_{j, x}^{\mathrm{ls}}$ from Theorem 7.7 requires the evaluation of $L_{2}(\Gamma)$-scalar products. For general parametrizations $\kappa_{q}$, these scalar products cannot be evaluated exactly, and therefore have to be approximated using numerical quadrature. Theorem 6.1 shows that if the quadrature is organized such that it causes an $L_{2}(\Gamma)$-error $\lesssim 2^{-\tilde{d} j}$ in the resulting approximate wavelet, then all results concerning cancellation properties and the generation of Riesz bases remain valid. 
8. Numerical example. We consider a 2-dimensional Lipschitz manifold $\Gamma=$ $\cup_{i=1}^{4} \overline{\Gamma_{q}} \subset \mathbb{R}^{3}$ as illustrated in Figure 8.1, which, together with its parametrization that satisfies $(\mathcal{M})$, is defined as follows. Let $P$ be a tetrahedron in $\mathbb{R}^{3}$, with vertices on the unit sphere, geometric centroid in $(0,0,0)$, and one of its four facets $F_{1}, \ldots, F_{4}$, say $F_{4}$, parallel to and below the $x_{3}=0$ plane. Let $\square$ be the interior of a reference 2 -simplex

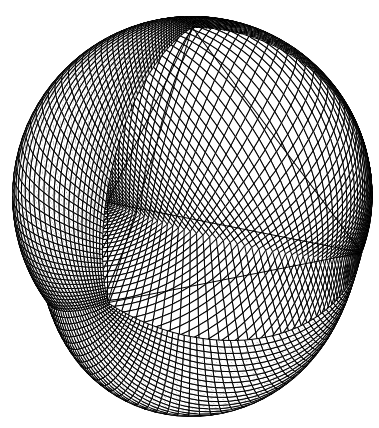

FIG. 8.1. The manifold $\Gamma$, excluding one of the patches $F_{1}, F_{2}$, or $F_{3}$, and the tetrahedron $P$.

in $\mathbb{R}^{2}$, with $\operatorname{vol}(\square)=1$, and for $1 \leq q \leq 4$, let $B_{q}: \square \rightarrow F_{q}$ some affine bijection. The parametrizations $\kappa_{q}: \square \rightarrow \Gamma_{q}$ are defined by $\kappa_{q}(z)=B_{q}(z) /\left\|B_{q}(z)\right\|$ for $1 \leq q \leq 3$, and by $\kappa_{4}(z)=B_{q}(z) /\left\|B_{q}(z)\right\|-\frac{27}{4}\left(0,0, \lambda_{1}(z) \lambda_{2}(z) \lambda_{3}(z)\right)$, where $\left(\lambda_{1}(z), \lambda_{2}(z), \lambda_{3}(z)\right)$ are the barycentric coordinates of $z$ with respect to $\square$. So without the perturbation by this cubic bubble, $\Gamma$ would be the unit sphere. We added this perturbation term so that $J: x \mapsto\left|\partial \kappa_{q}\left(\kappa_{q}^{-1}(x)\right)\right|$ when $x \in \Gamma_{q}$, cannot be extended to a continuous function on $\Gamma$. This means that constructions based on ignoring the Jacobian determinants will yield wavelets of which those that have supports that intersect an interface between $\Gamma_{4}$ and one of the three other patches have no cancellation properties. Furthermore, in view of our discussion at the beginning of $\S 6$, note that $z \mapsto\left|\partial \kappa_{q}(z)\right|$ are not constant functions.

We consider two examples of collections $\Phi_{j}^{\square}, \tilde{\Phi}_{j}^{\square}, \Theta_{j}^{\square}, \Xi_{j}^{\square}, \Lambda_{j}^{\square}, \tilde{\Lambda}_{j}^{\square}$, both based on the construction of finite element wavelets from [14]. With $\tau_{0}=\{\square\}$, let $\tau_{0}, \tau_{1}, \ldots$ be the sequence of triangulations of $\square$ with $\tau_{j+1}$ generated from $\tau_{j}$ by a uniform dyadic refinement, let $V_{j}$ be the set of vertices of all $T \in \tau_{j}$, and for $p \in \mathbb{N}_{>0}$, let $S_{j}^{p}=C(\bar{\square}) \cap \prod_{T \in \tau_{j}} P_{p}(T)$.

Let $\boldsymbol{\Phi}=\left(\boldsymbol{\phi}_{\alpha}\right)_{\alpha \in \boldsymbol{I}}, \tilde{\boldsymbol{\Phi}}=\left(\tilde{\boldsymbol{\phi}}_{\alpha}\right)_{\alpha \in \boldsymbol{I}}, \boldsymbol{\Theta}=\left(\boldsymbol{\theta}_{\alpha}\right)_{\alpha \in \boldsymbol{I}}, \boldsymbol{\Xi}=\left(\boldsymbol{\xi}_{\alpha}\right)_{\alpha \in \boldsymbol{J}}$, and $\boldsymbol{\Lambda}=\left(\boldsymbol{\lambda}_{\alpha}\right)_{\alpha \in \boldsymbol{I}}$, $\tilde{\boldsymbol{\Lambda}}=\left(\tilde{\boldsymbol{\lambda}}_{\alpha}\right)_{\alpha \in \boldsymbol{I}}$ be collections in $C(\bar{\square})$ or $C(\bar{\square})^{\prime}$, respectively, where both the index sets $\boldsymbol{I}$ and $\boldsymbol{J}$, and all collections of functions or functionals are symmetric in the barycentric coordinates. For $j \in \mathbb{N}$, and with, for each $T \in \tau_{j}, B_{T}: \square \rightarrow T$ being some arbitrary affine bijection, let $I_{j}^{\square}=\cup_{T \in \tau_{j}} B_{T}(\boldsymbol{I}), J_{j}^{\square}=\cup_{T \in \tau_{j}} B_{T}(\boldsymbol{J})$, and for $x \in \bar{\square}$, let $k_{j}^{\square}(x):=\#\left\{T \in \tau_{j}: x \in \bar{T}\right\}$. The 'local' index sets $\boldsymbol{I}$ and $\boldsymbol{J}$ will be chosen such that $J_{j}^{\square}=I_{j+1}^{\square} \backslash I_{j}^{\square}$. We define the collections $\Phi_{j}^{\square}:=\left(\phi_{j, x}^{\square}\right)_{x \in I_{j}^{\square}}$, and analogously $\tilde{\Phi}_{j}^{\square}, \Theta_{j}^{\square}$ and $\Xi_{j}^{\square}=\left(\xi_{j, y}^{\square}\right)_{y \in J_{j}^{\square}}$, and the collections $\Lambda_{j}^{\square}=\left(\lambda_{j, x}^{\square}\right)_{x \in I_{j}^{\square}}$, and analogously $\tilde{\Lambda}_{j}^{\square}$, by

$$
\phi_{j, x}^{\square}(z)=2^{j} k_{j}^{\square}(x)^{-\frac{1}{2}}\left\{\begin{array}{cl}
\phi_{B_{T}^{-1}(x)}\left(B_{T}^{-1}(z)\right) & \text { if } x, z \in T \in \tau_{j}, \\
0 & \text { elsewhere, }
\end{array}\right.
$$


and

$$
\lambda_{j, x}^{\square}(u)=2^{-j} k_{j}^{\square}(x)^{\frac{1}{2}} \lambda_{B_{T}^{-1}(x)}\left(u \circ B_{T}\right) \quad \text { when } x \in \bar{T} \text { for some } T \in \tau_{j},
$$

respectively. The 'local' collections will be designed such that the 'global' collections given by (8.1) or (8.2) are well-defined, where moreover all functions are continuous. Furthermore, it will hold that $\langle\boldsymbol{\Phi}, \boldsymbol{\Lambda}\rangle_{L_{2}(\square)}=\langle\tilde{\boldsymbol{\Phi}}, \tilde{\boldsymbol{\Lambda}}\rangle_{L_{2}(\square)}=\langle\boldsymbol{\Theta}, \tilde{\boldsymbol{\Phi}}\rangle_{L_{2}(\square)}=I d$, so that $\left\langle\Phi_{j}^{\square}, \Lambda_{j}^{\square}\right\rangle_{L_{2}(\square)}=\left\langle\tilde{\Phi}_{j}^{\square}, \tilde{\Lambda}_{j}^{\square}\right\rangle_{L_{2}(\square)}=\left\langle\Theta_{j}^{\square}, \tilde{\Phi}_{j}^{\square}\right\rangle_{L_{2}(\square)}=I d$, and all other conditions imposed in $\S 3$ will be also satisfied with parameters $\gamma=\tilde{\gamma}=\frac{3}{2}, d=2$, and either $\tilde{d}=2$ or $\tilde{d}=3$. However, in both our examples $\mathcal{S}(\boldsymbol{\Theta}) \neq \mathcal{S}(\boldsymbol{\Phi})$, so that the basis transformation from single-scale to multi-scale basis will not have a linear complexity.

In our example for $\tilde{d}=2$, we have $\boldsymbol{I}=V_{0}, \boldsymbol{J}=V_{1} \backslash V_{0}, \boldsymbol{\Phi}=\tilde{\boldsymbol{\Phi}}$ with $\mathcal{S}(\boldsymbol{\Phi})=S_{0}^{1}$, and $\mathcal{S}(\boldsymbol{\Theta} \cup \boldsymbol{\Xi})=S_{1}^{1}$. The collections were introduced in [14, §4.5.1], and they are determined by the illustrations in Figure 8.2 by using symmetry in the barycentric coordinates. The collection $\boldsymbol{\Lambda}=\tilde{\boldsymbol{\Lambda}}$ is just the set of function evaluations on $V_{0}$. Note

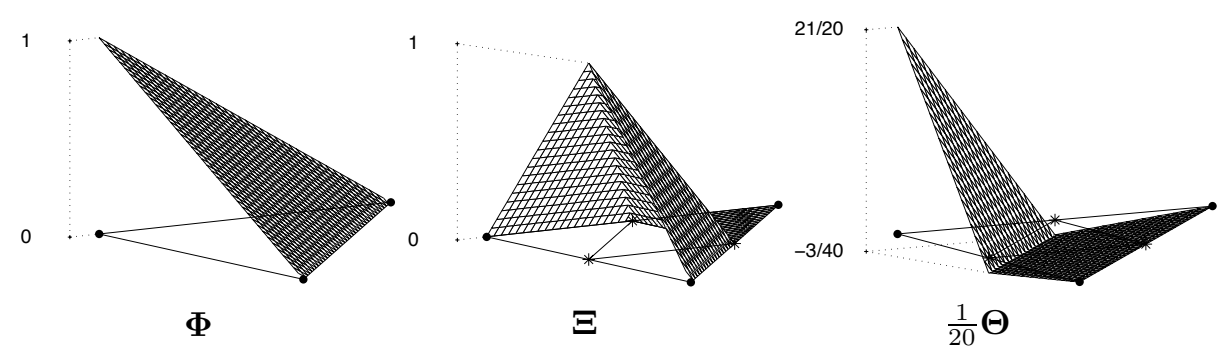

FIG. 8.2. "Local building blocks" for $\tilde{d}=2$.

that $I_{j}^{\square}=V_{j}, \mathcal{S}\left(\Phi_{j}^{\square}\right)=\mathcal{S}\left(\tilde{\Phi}_{j}^{\square}\right)=S_{j}^{1}$. So, in particular, for the resulting collection $\Phi_{j}$ on $\Gamma$, we have $\mathcal{S}\left(\Phi_{j}\right)=C(\Gamma) \cap \prod_{q=1}^{4} \kappa_{q}\left(S_{j}^{1}\right)$.

In our example for $\tilde{d}=3$, we have $\boldsymbol{I}=V_{1}, \boldsymbol{J}=V_{2} \backslash V_{1}, \mathcal{S}(\boldsymbol{\Phi})=S_{1}^{1}, \mathcal{S}(\tilde{\boldsymbol{\Phi}})=S_{0}^{2}$, and $\mathcal{S}(\boldsymbol{\Theta} \cup \boldsymbol{\Xi})=S_{2}^{1}$. The collections we use are modifications of those introduced in [14, §4.5.4], and yield better conditioned wavelet bases. They are illustrated in Figure 8.3, and their derivation and precise definition will be presented in [17], together with examples for other values of $d$ and $\tilde{d}$. The collection $\boldsymbol{\Lambda}$ is the set of function evaluations on $V_{1}$. Writing $\tilde{\boldsymbol{\Phi}}$ as an invertible $6 \times 6$ matrix $\mathbf{B}$ applied to the nodal basis of $S_{0}^{2}, \tilde{\boldsymbol{\Lambda}}$ is obtained by applying $\mathbf{B}^{-T}$ to the collection of function evaluations on $V_{1}$. Note that $I_{j}^{\square}=V_{j+1}, \mathcal{S}\left(\Phi_{j}^{\square}\right)=S_{j+1}^{1}$ and $\mathcal{S}\left(\tilde{\Phi}_{j}^{\square}\right)=S_{j}^{2}$. So for the resulting collections $\Phi_{j}$ and $\tilde{\Phi}_{j}$ on $\Gamma$, we have $\mathcal{S}\left(\Phi_{j}\right)=C(\Gamma) \cap \prod_{q=1}^{4} \kappa_{q}\left(S_{j+1}^{1}\right)$ and $\mathcal{S}\left(\tilde{\Phi}_{j}\right)=C(\Gamma) \cap \prod_{q=1}^{4} \kappa_{q}\left(S_{j}^{2}\right)$, respectively.

We have implemented the approximate wavelet constructions from $\S 7.2$ and $\S 7.2$, that away from the patch interfaces both yield the approximate wavelets from the collection $\Psi_{j}^{(0)}=\Xi_{j}-\left\langle\left\langle\Xi_{j}, \tilde{\Phi}_{j}\right\rangle\right\rangle_{0}\left\langle\left\langle\Theta_{j}, \tilde{\Phi}_{j}\right\rangle\right\rangle_{0}^{-1} \Theta_{j}$ obtained by ignoring the Jacobian determinants. The pull-backs of these wavelets to the parameter domain are illustrated in Figure 8.4, which functions are thus continuous piecewise linear with respect to the indicated triangulation.

With the approach from $\$ 7.2$, wavelets along the patch interfaces are taken from the collection $\Psi_{j}^{\mathrm{Jc}}:=\Xi_{j}-\left\langle\Xi_{j}, \tilde{\Phi}_{j}\right\rangle_{L_{2}(\Gamma)}\left[\sum_{k=0}^{\tilde{d}-1}\left(I d-\mathbf{D}_{j}^{-1}\left\langle\Theta_{j}, \tilde{\Phi}_{j}\right\rangle_{L_{2}(\Gamma)}\right)^{k} \mathbf{D}_{j}^{-1}\right] \Theta_{j}$, where $\mathbf{D}_{j}:=\operatorname{diag}\left\langle\Theta_{j}, \tilde{\Phi}_{j}\right\rangle_{L_{2}(\Gamma)}$. Illustrations of the, naturally joined, patchwise pullbacks of these wavelets can be found in Figure 8.5. For $j=0$, the Neumann se- 


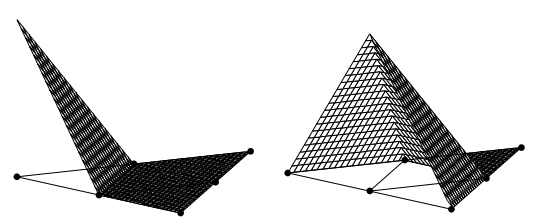

$\Phi$

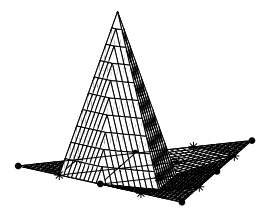

$\Xi$

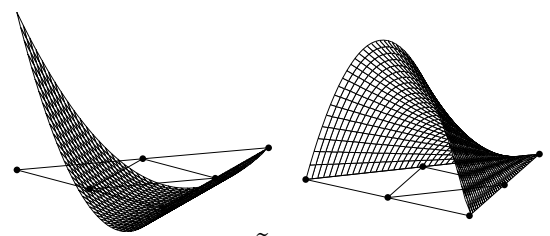

$\tilde{\Phi}$

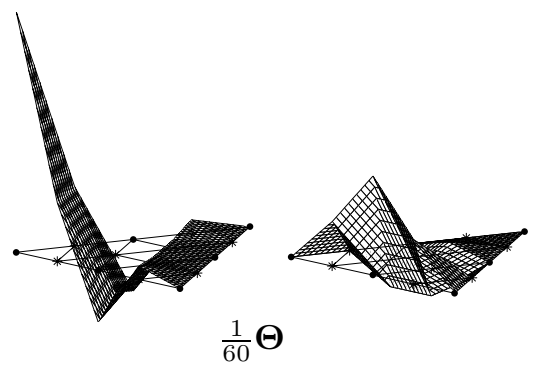

FIG. 8.3. "Local building blocks" for $\tilde{d}=3$.
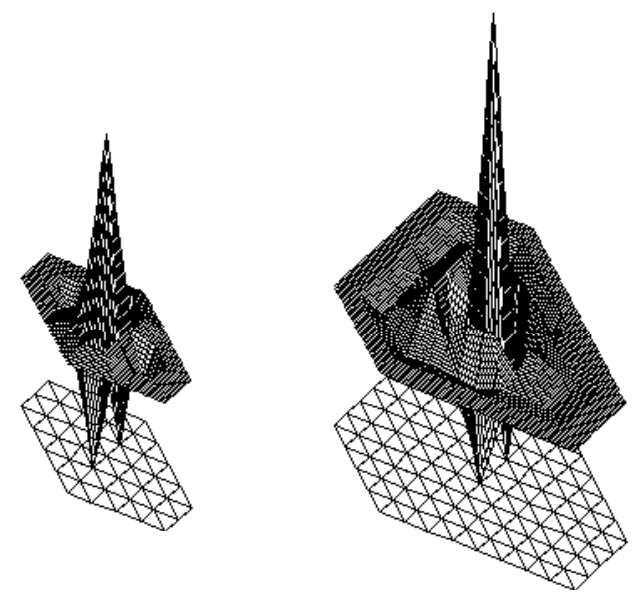

FIG. 8.4. Wavelets $\psi_{j, x}^{(0)}$ away from the patch interfaces for $\tilde{d}=2$ and $\tilde{d}=3$ (one of the two different types), and their supports in terms of the underlying triangulation.

ries does not converge, and as a consequence $\sum_{k=0}^{\tilde{d}-1}\left(I d-\mathbf{D}_{j}^{-1}\left\langle\Theta_{j}, \tilde{\Phi}_{j}\right\rangle_{L_{2}(\Gamma)}\right)^{k} \mathbf{D}_{j}^{-1}$ provides a very poor approximation for $\left\langle\Theta_{j}, \tilde{\Phi}_{j}\right\rangle_{L_{2}(\Gamma)}^{-1}$. We redefined $\breve{\Psi}_{0}:=\Xi_{0}-$ $\left\langle\Xi_{j}, \tilde{\Phi}_{j}\right\rangle_{L_{2}(\Gamma)}\left\langle\Theta_{j}, \tilde{\Phi}_{j}\right\rangle_{L_{2}(\Gamma)}^{-1} \Theta_{0}$.

For the construction from $\S 7.2$, for each $x \in J_{j} \backslash J_{j}^{\circ}$ we have to specify a subspace $A_{j, x} \subset \mathcal{S}\left(\Phi_{j+1}\right)$ that defines $\psi_{j, x}^{\text {ls }}$ via

$$
\underset{\psi_{j, x}^{\mathrm{ls}} \in \psi_{j, x}+A_{j, x}}{\operatorname{argmin}}\left\{\left\|\psi_{j, x}^{\mathrm{ls}}-\hat{\psi}_{j, x}\right\|_{L_{2}(\Gamma)}: \psi_{j, x}^{\mathrm{ls}} \perp_{L_{2}(\Gamma)} \mathbb{P}_{\tilde{d}-2}(\Gamma)\right\} .
$$

For $\tilde{d}=2$, we take $A_{j, x}=\mathcal{S}\left(\left\{\xi_{j, x}\right\}\right)$, so that $\psi_{j, x}^{\mathrm{ls}}=\hat{\psi}_{j, x}+\alpha \xi_{j, x}$ with $\alpha$ such that $\int_{\Gamma} \psi_{j, x}^{\mathrm{ls}}=0$. For $\tilde{d}=3$, we take $A_{j, x}=\mathcal{S}\left(\left\{\phi_{j+1, y}: \operatorname{supp} \phi_{j+1, y} \subset \operatorname{supp} \hat{\psi}_{j, x}\right\}\right)$, which space turns out to be sufficiently large so that the constrained minimization problem has a solution $\psi_{j, x}^{\mathrm{ls}}$, with $\left\|\psi_{j, x}^{\mathrm{ls}}-\hat{\psi}_{j, x}\right\|_{L_{2}(\Gamma)} \lesssim 2^{-j}$. The, naturally joined, 

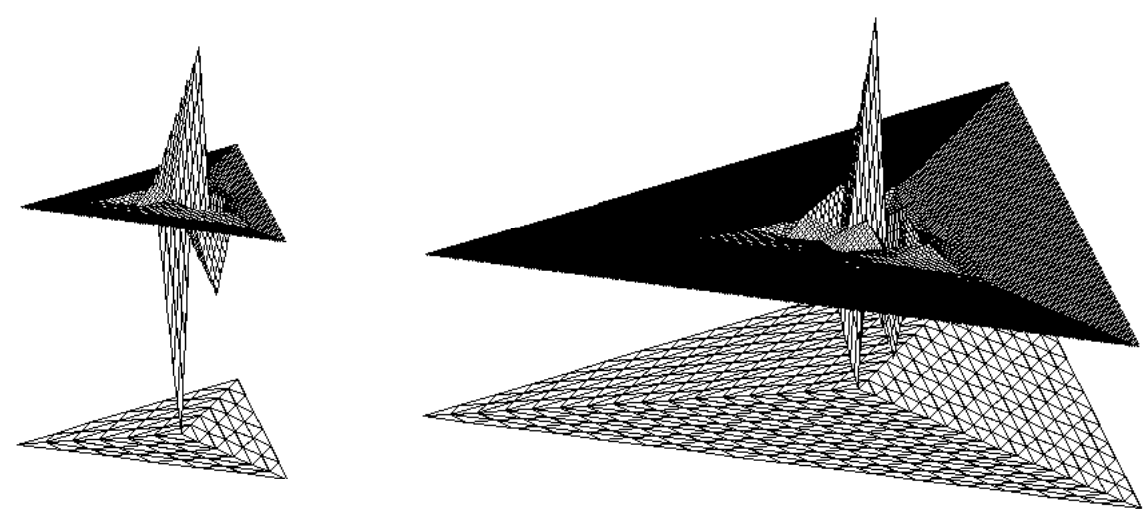

FIG. 8.5. Wavelets $\psi_{4, x}^{\mathrm{Jc}}$ for $\tilde{d}=2$ and $\psi_{3, x}^{\mathrm{Jc}}$ for $\tilde{d}=3$ near the "north pole", and their supports in terms of the underlying triangulation.

patchwise pull-backs of the resulting $\psi_{j, x}^{\mathrm{ls}}$ are illustrated in Figure 8.6. By definition they have the same supports as the corresponding $\psi_{j, x}^{(0)}$ that one obtains by ignoring the Jacobian determinants also along the interfaces. As with the Jacobi iteration
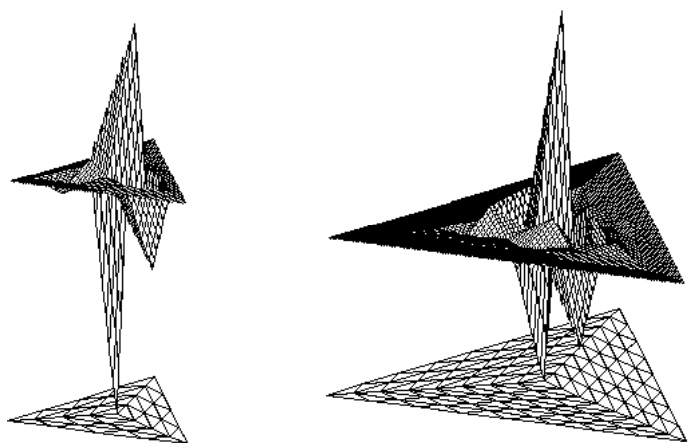

FIG. 8.6. Wavelets $\psi_{4, x}^{\mathrm{ls}}$ for $\tilde{d}=2$ and $\psi_{3, x}^{\mathrm{ls}}$ for $\tilde{d}=3$ near the "north pole", and their supports in terms of the underlying triangulation.

approach, for $j=0$, we redefined $\breve{\Psi}_{0}:=\Xi_{0}-\left\langle\Xi_{j}, \tilde{\Phi}_{j}\right\rangle_{L_{2}(\Gamma)}\left\langle\Theta_{j}, \tilde{\Phi}_{j}\right\rangle_{L_{2}(\Gamma)}^{-1} \Theta_{0}$.

With

$$
\kappa_{\Sigma,\|\cdot\|}:=\sup _{0 \neq \mathbf{c}=\left(c_{\sigma}\right)_{\sigma \in \Sigma}} \frac{\left\|\sum_{\sigma \in \Sigma} c_{\sigma} \frac{\sigma}{\|\sigma\|}\right\|^{2}}{\|\mathbf{c}\|^{2}} / \inf _{0 \neq \mathbf{c}=\left(c_{\sigma}\right)_{\sigma \in \Sigma}} \frac{\left\|\sum_{\sigma \in \Sigma} c_{\sigma} \frac{\sigma}{\|\sigma\|}\right\|^{2}}{\|\mathbf{c}\|^{2}},
$$

and, for the obtained collections $\breve{\Psi}_{j}$ of approximate wavelets using either the approach from $\S 7.2$ (Jacobi approximation along the interfaces) or that of $\S 7.2$ (least squares approximation along the interfaces), with

$$
\breve{\Psi}^{(\ell)}:= \begin{cases}\Phi_{0} \cup \cup_{k=0}^{\ell-1} \breve{\Psi}_{k} & \text { when } \tilde{d}=2, \\ \Phi_{0} \cup \cup_{k=0}^{\ell-2} \breve{\Psi}_{k} & \text { when } \tilde{d}=3,\end{cases}
$$

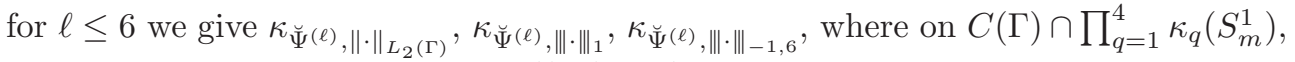
$\|u\|_{-1, m}:=\sup _{v \in C(\Gamma) \cap \prod_{q=1}^{4} \kappa_{q}\left(S_{m}^{1}\right)} \frac{\left|\langle u, v\rangle_{L_{2}(\Gamma)}\right|}{\|v\|_{1}}$. The uniform boundedness in $\|\cdot\|_{1}$ of 
the $L_{2}(\Gamma)$-orthogonal projector onto $C(\Gamma) \cap \prod_{q=1}^{4} \kappa_{q}\left(S_{m}^{1}\right)$, which is a consequence of Theorem 5.3, shows that $\|\cdot\|_{-1} \bar{\sim}\|\cdot\|_{-1, m}$ on $C(\Gamma) \cap \prod_{q=1}^{4} \kappa_{q}\left(S_{\ell}^{1}\right)$ uniformly in $\ell \leq m$. Recall that for both $\tilde{d}=2$ and $\tilde{d}=3, \breve{\Psi}^{(\ell)}$ is a basis for $C(\Gamma) \cap \prod_{q=1}^{4} \kappa_{q}\left(S_{\ell}^{1}\right)$. Since the functions from $\Phi_{0}$, and for $\tilde{d}=2$, from $\breve{\Psi}_{0}$ and $\breve{\Psi}_{1}$, and for $\tilde{d}=3$, from $\breve{\Psi}_{0}$ have global supports anyway, for computing the condition numbers we replaced each of these collections by orthonormalized versions by multiplying them by the inverse of the square root of their mass matrix with respect to either $\langle\cdot, \cdot\rangle_{L_{2}(\Gamma)},\left\langle\langle\cdot, \cdot\rangle_{1}\right.$ or $\langle\langle\cdot, \cdot\rangle\rangle_{-1,6}$, the latter being the scalar product corresponding to the norm $\|\cdot\|_{-1,6}$. The results are presented in Tables 8.1 and 8.2. Although it turns out that unfortunately,

TABLE 8.1

Condition numbers for $\tilde{d}=2$.

\begin{tabular}{|c|c|c|c|c|c|c|}
\hline & \multicolumn{3}{|c|}{ Jacobi approximation } & \multicolumn{3}{|c|}{ least squares approximation } \\
\hline$\ell$ & $\kappa_{\Psi}^{(\ell)},\|\cdot\|_{L_{2}(\Gamma)}$ & $\kappa_{\breve{\Psi}(\ell),\|\cdot\|_{1}}$ & $\kappa_{\breve{\Psi}^{(\ell)},\|\cdot\| \|_{-1,6}}$ & $\kappa_{\breve{\Psi}(\ell)},\|\cdot\|_{L,(\Gamma)}$ & $\kappa_{\breve{\Psi}^{(\ell)},\|\cdot\|_{1}}$ & $\kappa_{\breve{\Psi}^{(\ell)},\|\cdot\| \|_{-1,6}}$ \\
\hline 1 & $1.35 e 0$ & $2.30 e 0$ & $3.06 e 0$ & $1.35 e 0$ & $2.30 e 0$ & $3.09 e 0$ \\
\hline 2 & $1.45 e 0$ & $7.86 e 0$ & $8.65 e 0$ & $1.82 e 0$ & $7.64 e 0$ & $1.09 e 1$ \\
\hline 3 & $1.75 e 1$ & $4.82 e 1$ & $2.79 e 1$ & $2.42 e 1$ & $6.84 e 1$ & $3.41 e 1$ \\
\hline 4 & $1.79 e 1$ & $6.51 e 1$ & $4.53 e 1$ & $2.46 e 1$ & $9.06 e 1$ & $5.21 e 1$ \\
\hline 5 & $1.79 e 1$ & $7.66 e 1$ & $6.20 e 1$ & $2.46 e 1$ & $1.06 e 2$ & $6.69 e 1$ \\
\hline 6 & $1.79 e 1$ & $8.25 e 1$ & $7.34 e 1$ & $2.46 e 1$ & $1.14 e 2$ & $7.84 e 1$ \\
\hline
\end{tabular}

TABLE 8.2

Condition numbers for $\tilde{d}=3$.

\begin{tabular}{|c||c|c|c||c|c|c|}
\hline & \multicolumn{3}{|c||}{ Jacobi approximation } & \multicolumn{3}{c|}{ least squares approximation } \\
\hline$\ell$ & $\kappa_{\Psi^{(\ell)},\|\cdot\|_{L_{2}(\Gamma)}}$ & $\kappa_{\Psi^{(}(\ell),\|\cdot \cdot\|_{1}}$ & $\kappa_{\Psi^{(\ell)},\|\cdot\| \|_{-1,6}}$ & $\kappa_{\breve{\Psi}^{(\ell)},\|\cdot\|_{L_{2}(\Gamma)}}$ & $\kappa_{\breve{\Psi}^{(\ell)},\|\cdot \cdot\|_{1}}$ & $\kappa_{\breve{\Psi}^{(\ell)},\|\cdot\|_{-1,6}}$ \\
\hline 2 & $2.80 e 0$ & $1.26 e 1$ & $4.18 e 1$ & $2.80 e 0$ & $1.26 e 1$ & $4.22 e 0$ \\
\hline 3 & $1.36 e 1$ & $5.47 e 1$ & $3.36 e 1$ & $1.85 e 1$ & $8.51 e 1$ & $3.82 e 1$ \\
\hline 4 & $1.81 e 1$ & $7.88 e 1$ & $5.76 e 1$ & $2.34 e 1$ & $1.19 e 2$ & $6.86 e 1$ \\
\hline 5 & $1.97 e 1$ & $8.83 e 1$ & $8.00 e 1$ & $2.44 e 1$ & $1.32 e 2$ & $9.65 e 1$ \\
\hline 6 & $2.03 e 1$ & $9.20 e 1$ & $9.64 e 1$ & $2.47 e 1$ & $1.36 e 2$ & $1.34 e 2$ \\
\hline
\end{tabular}

in particular in the $\|\cdot\|_{-1,6}$-norm, the condition numbers haven't really stabilized, we stopped our computations at $\ell=6$ since mainly due to the normalization of the wavelets, in particular with respect to the $\|\cdot\|_{-1,6}$-norm, on higher levels they become too time consuming.

Appendix A. Proof of Theorem 6.1. The proof consists of steps (I)-(VI). Although basically (V) and (VI) can be found in [20, Theorem 3.1], which in turn was based on [24, Appendix], for convenience we include a complete proof.

(I) Since, as shown in Proposition 5.4, for $j \geq j_{0}$ large enough, $\Psi_{j}$ is a uniform $L_{2}(\Gamma)$-Riesz system, and by Condition (iii),

$$
\begin{aligned}
& \left\|\left\langle\breve{\Psi}_{j}, \breve{\Psi}_{j}\right\rangle_{L_{2}(\Gamma)}-\left\langle\Psi_{j}, \Psi_{j}\right\rangle_{L_{2}(\Gamma)}\right\| \\
& =\left\|\left\langle\breve{\Psi}_{j}-\Psi_{j}, \Psi_{j}\right\rangle_{L_{2}(\Gamma)}+\left\langle\Psi_{j}, \breve{\Psi}_{j}-\Psi_{j}\right\rangle_{L_{2}(\Gamma)}+\left\langle\breve{\Psi}_{j}-\Psi_{j}, \breve{\Psi}_{j}-\Psi_{j}\right\rangle_{L_{2}(\Gamma)}\right\| \lesssim \omega^{j},
\end{aligned}
$$

we infer that, possibly for a larger value of $j_{0}$, for $j \geq j_{0}, \breve{\Psi}_{j}$ is a uniform $L_{2}(\Gamma)$-Riesz system. 
(II) Next we investigate whether $\mathcal{S}\left(\Phi_{j}\right)+\mathcal{S}\left(\breve{\Psi}_{j}\right)$ is a uniformly $L_{2}(\Gamma)$-stable twolevel decomposition of $\mathcal{S}\left(\Phi_{j+1}\right)$ :

Proposition A.1. Let $V, W$ be subspaces of a Hilbert space $H$. Then equivalent are

(a) $H=V+W$ and $\alpha:=\sup _{0 \neq v \in V, 0 \neq w \in W} \frac{|\langle v, w\rangle|}{\|v\|\|w\|}<1$,

(b) There exists a bounded projector $Q: H \rightarrow H$ with $\Im(Q)=V$ and $\Im(I d-Q)=W$. Moreover, $\|Q\|=\left(1-\alpha^{2}\right)^{-\frac{1}{2}}$.

Now let (a), or equivalently (b), be satisfied, and let $\breve{W}$ be another subspace of $H$ for which there exists a linear mapping $R: \breve{W} \rightarrow W$ with $\|I d-R\|<\frac{1-\alpha}{1+\alpha}$. Then

$$
\breve{\alpha}:=\sup _{0 \neq v \in V, 0 \neq \breve{w} \in \breve{W}} \frac{|\langle v, \breve{w}\rangle|}{\|v\|\|\breve{w}\|} \leq \alpha+(1+\alpha)\|I d-R\|<1 .
$$

With $\breve{Q}: H \rightarrow H$ being the bounded projector with $\Im(\breve{Q})=V$ and $\Im(I d-\breve{Q})=\breve{W}$, it holds that

$$
\|Q-\breve{Q}\| \leq \frac{\|I d-R\|}{\left(1-\alpha^{2}\right)^{\frac{1}{2}}\left(1-\breve{\alpha}^{2}\right)^{\frac{1}{2}}} .
$$

Proof. If (a) is valid, then $H=V \oplus W$, and so there exists a projector $Q$ with $\Im(Q)=V$ and $\Im(I d-Q)=W$. For any $u \in H$, we have

$$
\begin{aligned}
\|u\|^{2} & =\|Q u+(I d-Q) u\|^{2} \geq\|Q u\|^{2}-2|\langle Q u,(I d-Q) u\rangle|+\|(I d-Q) u\|^{2} \\
& \geq\|Q u\|^{2}-2 \alpha\|Q u\|\|(I d-Q) u\|+\|(I d-Q) u\|^{2} \geq\left(1-\alpha^{2}\right)\|Q u\|^{2},
\end{aligned}
$$

or $\|Q\| \leq\left(1-\alpha^{2}\right)^{-\frac{1}{2}}$.

Now let (b) be valid. Suppose there exist nonzero $v \in V, w \in W$ such that $\mu:=\frac{|\langle v, w\rangle|}{\|v\|\|w\|}>\left(1-\|Q\|^{-2}\right)^{\frac{1}{2}}$. Then there exist nonzero $v \in V, w \in W$ with $\langle v, w\rangle=$ $-\mu\|v\|\|w\|$, moreover which can be chosen such that $\|w\|=-\mu\|v\|$. From

$$
\|Q\|^{-2}\|v\|^{2} \leq\|v+w\|^{2}=\|v\|^{2}+2\langle v, w\rangle+\|w\|^{2}=\left(1-\mu^{2}\right)\|v\|^{2}
$$

we conclude a contradiction, so that $\sup _{0 \neq v \in V, 0 \neq w \in W} \frac{|\langle v, w\rangle|}{\|v\|\|w\|} \leq\left(1-\|Q\|^{-2}\right)^{\frac{1}{2}}$.

Now let (a) or, equivalently, (b) be valid, and let $\breve{W}$ be a subspace as in the proposition. For any $v \in V, \breve{w} \in \breve{W}$,

$$
\begin{aligned}
|\langle v, \breve{w}\rangle| & =|\langle v, R \breve{w}\rangle+\langle v,(I d-R) \breve{w}\rangle| \leq \alpha\|v\|\|R \breve{w}\|+\|v\|\|(I d-R) \breve{w}\| \\
& \leq \alpha\|v\|\|\breve{w}\|+(1+\alpha)\|v\|\|(I d-R) \breve{w}\| \leq(\alpha+(1+\alpha)\|I d-R\|)\|v\|\|\breve{w}\|,
\end{aligned}
$$

showing the statement about $\breve{\alpha}$. The last statement follows from $\|Q\|=\left(1-\alpha^{2}\right)^{-\frac{1}{2}}$, $\|I d-\breve{Q}\|=\left(1-\breve{\alpha}^{2}\right)^{-\frac{1}{2}}$, and $Q-\breve{Q}=Q(I d-R)(I d-\breve{Q})$ by $Q R=0$ and $Q \breve{Q}=\breve{Q}$, $\mathbf{\square}$

Returning to the proof of Theorem 6.1, with, for $j \geq j_{0}, Q_{j}^{(j+1)}:=\left.Q_{j}\right|_{\mathcal{S}\left(\Phi_{j+1}\right)}$, it holds that $\Im\left(Q_{j}^{(j+1)}\right)=\mathcal{S}\left(\Phi_{j}\right)$ and $\Im\left(I d-Q_{j}^{(j+1)}\right)=\mathcal{S}\left(\Psi_{j}\right)$. Setting $R_{j}: \mathcal{S}\left(\breve{\Psi}_{j}\right) \rightarrow$ $\mathcal{S}\left(\Psi_{j}\right): \mathbf{c}_{j}^{T} \breve{\Psi}_{j} \mapsto \mathbf{c}_{j}^{T} \Psi_{j}$, by Condition (iii) and (I) we have

$$
\left\|\left(I d-R_{j}\right) \mathbf{c}_{j}^{T} \breve{\Psi}_{j}\right\|_{L_{2}(\Gamma)}=\left\|\mathbf{c}_{j}^{T}\left(\breve{\Psi}_{j}-\Psi_{j}\right)\right\|_{L_{2}(\Gamma)} \leq\|\mathbf{c}\|\left\|\breve{\Psi}_{j}-\Psi_{j}\right\|_{L_{2}(\Gamma)} \lesssim\left\|\mathbf{c}_{j}^{T} \breve{\Psi}_{j}\right\|_{L_{2}(\Gamma)} \omega^{j}
$$


or $\left\|I d-R_{j}\right\|_{L_{2}(\Gamma) \rightarrow L_{2}(\Gamma)} \lesssim \omega^{j}$. From Proposition A.1 we conclude that, possibly for a larger value of $j_{0}$, for $j \geq j_{0}$ there exists a uniformly $L_{2}(\Gamma)$-bounded projector $\breve{Q}_{j}^{(j+1)}: \mathcal{S}\left(\Phi_{j+1}\right) \rightarrow \mathcal{S}\left(\Phi_{j+1}\right)$ with

$$
\Im\left(\breve{Q}_{j}^{(j+1)}\right)=\mathcal{S}\left(\Phi_{j}\right), \quad \Im\left(I d-\breve{Q}_{j}^{(j+1)}\right)=\mathcal{S}\left(\breve{\Psi}_{j}\right),
$$

and

$$
\left\|Q_{j}^{(j+1)}-\breve{Q}_{j}^{(j+1)}\right\|_{L_{2}(\Gamma) \rightarrow L_{2}(\Gamma)} \lesssim \omega^{j} .
$$

(III) By Condition (i), $\breve{\Psi}_{j}$ is uniformly local. Since furthermore, as shown in (I), $\breve{\Psi}_{j}$ is a uniform $L_{2}(\Gamma)$-Riesz system, that, by Condition (ii), has the uniform cancellation property of order $\tilde{d}$, for some $\tilde{\eta} \geq 0$ we have

$$
\begin{aligned}
& \left|\left\langle\mathbf{c}_{j}^{T} \breve{\Psi}_{j}, u\right\rangle_{L_{2}(\Gamma)}\right| \leq \sum_{x \in J_{j}}\left|c_{j, x}\right|\left|\left\langle\breve{\psi}_{j, x}, u\right\rangle_{L_{2}(\Gamma)}\right| \quad\left(\mathbf{c}_{j} \in \ell_{2}\left(J_{j}\right), u \in \mathcal{H}_{\tilde{d}}(\Gamma)\right) \\
& \lesssim 2^{-j \tilde{d}}\left\|\mathbf{c}_{j}\right\|\left[\sum_{x \in J_{j}} \sum_{q=1}^{M}\left\|u \circ \kappa_{q}\right\|_{H^{\tilde{d}}\left(B\left(\kappa_{q}^{-1}\left(\operatorname{supp} \breve{\psi}_{j, x} \cap \Gamma_{q}\right) ; \tilde{\eta} 2^{-j}\right) \cap \square\right)}^{2}\right)^{\frac{1}{2}} \lesssim 2^{-j \tilde{d}}\left\|\mathbf{c}_{j}^{T} \breve{\Psi}_{j}\right\|_{L_{2}(\Gamma)}\|u\|_{\tilde{d}},
\end{aligned}
$$

or

$$
\|\cdot\|_{-\tilde{d}} \lesssim 2^{-\tilde{d} j}\|\cdot\|_{L_{2}(\Gamma)} \quad \text { on } \mathcal{S}\left(\breve{\Psi}_{j}\right) .
$$

By the uniform $L_{2}(\Gamma)$-boundedness of $\breve{Q}_{j}^{(j+1)}$ for $j \geq j_{0}$, an direct consequence of the last result is that $\left\|I d-\breve{Q}_{j}^{(j+1)}\right\|_{0 \rightarrow-\tilde{d}} \lesssim 2^{-\tilde{d} j}$. By the Jackson estimate at the dual side (4.6), and the uniform $L_{2}(\Gamma)$-boundedness of $Q_{j}$ for $j \geq j_{0}$, we have $\left\|I d-Q_{j}^{(j+1)}\right\|_{0 \rightarrow-\tilde{d}} \leq\left\|I d-Q_{j}\right\|_{0 \rightarrow-\tilde{d}} \lesssim 2^{-\tilde{d} j}$, and so $\left\|Q_{j}^{(j+1)}-\breve{Q}_{j}^{(j+1)}\right\|_{0 \rightarrow-\tilde{d}} \lesssim 2^{-\tilde{d} j}$. By the extended Bernstein inequality Lemma $4.2,\left\|Q_{j}^{(j+1)}-\breve{Q}_{j}^{(j+1)}\right\|_{-\tilde{d} \rightarrow-\tilde{d}} \lesssim 1$, so that by interpolation using (A.1) we infer that

$$
\left\|Q_{j}^{(j+1)}-\breve{Q}_{j}^{(j+1)}\right\|_{s \rightarrow s} \lesssim \omega^{\left(1+\frac{s}{d}\right) j}, \quad(s \in[-\tilde{d}, 0]) .
$$

(IV) Knowing (A.3), we are ready to investigate the stability of the multi-level decomposition defined by the collections $\breve{\Psi}_{j}$. We are going to construct a projector $\breve{Q}_{j}$ defined on $\mathcal{H}_{s}(\Gamma)$ for some range of $s$, such that, for $j \geq j_{0}, \Im\left(\breve{Q}_{j}\right)=\mathcal{S}\left(\Phi_{j}\right)$ and $\Im\left(\breve{Q}_{j+1}-\breve{Q}_{j}\right)=\mathcal{S}\left(\breve{\Psi}_{j}\right)$.

By writing $Q_{j}=\sum_{k=j_{0}}^{j} Q_{k}-Q_{k-1}$, for any $s \in(-\min \{\tilde{\gamma}, \tilde{d}\}, \min \{\gamma, d\})$ and $u \in \mathcal{H}_{s}(\Gamma)$, Theorem 5.3 shows that $\left\|Q_{j} u\right\|_{s}^{2} \lesssim \sum_{k=j_{0}}^{j} 4^{s k}\left\|\left(Q_{k}-Q_{k-1}\right) u\right\|_{L_{2}(\Gamma)}^{2} \leq$ $\sum_{k=j_{0}}^{\infty} 4^{s k}\left\|\left(Q_{k}-Q_{k-1}\right) u\right\|_{L_{2}(\Gamma)}^{2} \lesssim\|u\|_{s}^{2}$ or $\left\|Q_{j}\right\|_{s \rightarrow s} \lesssim 1$.

For $\ell \geq j \geq j_{0}$, we define $\breve{Q}_{j}^{(\ell)}: \mathcal{S}\left(\Phi_{\ell}\right) \rightarrow \mathcal{S}\left(\Phi_{j}\right)$ by $\breve{Q}_{\ell}^{(\ell)}=I d$ and, for $j<\ell$, by $\breve{Q}_{j}^{(\ell)}=\breve{Q}_{j}^{(j+1)} \breve{Q}_{j+1}^{(\ell)}$. For some arbitrary, but fixed $t \in(-\min \{\tilde{\gamma}, \tilde{d}\}, 0]$, we set

$$
\rho_{j}^{(\ell)}:=\max _{j_{0} \leq k \leq j}\left\|Q_{k} \breve{Q}_{j}^{(\ell)}\right\|_{t \rightarrow t}, \quad \varepsilon_{j}:=\max _{j_{0} \leq k \leq j}\left\|Q_{k}\left(\breve{Q}_{j}^{(j+1)}-Q_{j}^{(j+1)}\right)\right\|_{t \rightarrow t} .
$$

From $Q_{k} \breve{Q}_{j}^{(\ell)}=Q_{k}\left(\breve{Q}_{j}^{(j+1)}-Q_{j}^{(j+1)}\right) \breve{Q}_{j+1}^{(\ell)}+Q_{k} \breve{Q}_{j+1}^{(\ell)}$, we find $\rho_{j}^{(\ell)} \leq\left(\varepsilon_{j}+1\right) \rho_{j+1}^{(\ell)}$, and so by $\rho_{\ell}^{(\ell)}=\max _{j_{0} \leq k \leq j}\left\|Q_{k}\right\|_{t \rightarrow t} \lesssim 1$, and $\varepsilon_{j} \lesssim \omega^{\left(1+\frac{t}{d}\right) j}$ by (A.3), we infer that

$$
\left\|\breve{Q}_{j}^{(\ell)}\right\|_{t \rightarrow t} \leq \rho_{j}^{(\ell)} \lesssim \prod_{k=j}^{\ell-1}\left(\varepsilon_{k}+1\right) \lesssim 1+\sum_{k=j}^{\ell-1} \varepsilon_{k} \lesssim 1,
$$


which thus hold uniformly in $j$ and $\ell$.

As a consequence of $I d=\sum_{j=j_{0}}^{\infty}\left(Q_{j}-Q_{j-1}\right)$ on $\mathcal{H}_{t}(\Gamma)$ by Theorem 5.3, we have $\operatorname{clos}_{H_{t}(\Gamma)} \cup_{j \geq j_{0}} \mathcal{S}\left(\Phi_{j}\right)=\mathcal{H}_{t}(\Gamma)$. Since for any $u \in \mathcal{H}_{t}(\Gamma), j \leq k \leq \ell$ and $u_{k} \in \mathcal{S}\left(\Phi_{k}\right)$, $\breve{Q}_{j}^{(\ell)} Q_{\ell} u=\breve{Q}_{j}^{(k)} u_{k}+\breve{Q}_{j}^{(\ell)} Q_{\ell}\left(u-u_{k}\right)$, from $\left\|\breve{Q}_{j}^{(\ell)} Q_{\ell}\right\|_{t \rightarrow t} \lesssim 1$ we infer that for any $j \geq j_{0}$, $\left(\breve{Q}_{j}^{(\ell)} Q_{\ell} u\right)_{\ell \geq j}$ is a Cauchy-sequence in $\mathcal{H}_{t}(\Gamma)$, and we set $\breve{Q}_{j} u=\lim _{\ell \rightarrow \infty} \breve{Q}_{j}^{(\ell)} Q_{\ell} u$. We conclude that $\breve{Q}_{j}: \mathcal{H}_{t}(\Gamma) \rightarrow \mathcal{H}_{t}(\Gamma)$ is uniformly bounded, with $\Im\left(\breve{Q}_{j}\right)=\mathcal{S}\left(\Phi_{j}\right)$ and $\Im\left(\breve{Q}_{j+1}-\breve{Q}_{j}\right)=\mathcal{S}\left(\breve{\Psi}_{j}\right)$

(V) Let $s \in(t, \min \{\gamma, d\})$. With $\breve{Q}_{j_{0}-1}:=0$, we are going to prove that

$$
\sum_{j=j_{0}}^{\infty} 4^{s j}\left\|\left(\breve{Q}_{j}-\breve{Q}_{j-1}\right) u\right\|_{L_{2}(\Gamma)}^{2} \lesssim\|u\|_{s}^{2}, \quad\left(u \in \mathcal{H}_{s}(\Gamma)\right) .
$$

For $u \in \mathcal{H}_{s}(\Gamma), w_{\ell}:=\left(Q_{\ell}-Q_{\ell-1}\right) u$, Theorem 6.1 shows that $u=\sum_{\ell=j_{0}}^{\infty} w_{\ell},\|u\|_{s}^{2} \bar{\sim}$ $\sum_{\ell=j_{0}}^{\infty} 4^{s \ell}\left\|w_{\ell}\right\|_{L_{2}(\Gamma)}^{2}$, and $\left\|w_{\ell}\right\|_{t} \lesssim 2^{t \ell}\left\|w_{\ell}\right\|_{L_{2}(\Gamma)}$. Since $\left\|\breve{Q}_{j}\right\|_{t \rightarrow 0} \lesssim 2^{-t j}$, which follows from $\left\|\breve{Q}_{j}\right\|_{t \rightarrow t} \lesssim 1$ and the extended Bernstein inequality Lemma 4.2 , and $\breve{Q}_{j}-\breve{Q}_{j-1}=$ 0 on $\mathcal{S}\left(\Phi_{j-1}\right)$, we arrive at

$$
\begin{aligned}
& \sum_{j=j_{0}}^{\infty} 4^{s j}\left\|\left(\breve{Q}_{j}-\breve{Q}_{j-1}\right) u\right\|_{L_{2}(\Gamma)}^{2}=\sum_{j=j_{0}}^{\infty} \sum_{\ell, \ell^{\prime}=j_{0}}^{\infty} 4^{s j}\left\langle\left(\breve{Q}_{j}-\breve{Q}_{j-1}\right) w_{\ell},\left(\breve{Q}_{j}-\breve{Q}_{j-1}\right) w_{\ell^{\prime}}\right\rangle_{L_{2}(\Gamma)} \\
& =\sum_{\ell, \ell^{\prime}=j_{0}}^{\infty} \sum_{j=j_{0}}^{\min \left\{\ell, \ell^{\prime}\right\}} 4^{s j}\left\langle\left(\breve{Q}_{j}-\breve{Q}_{j-1}\right) w_{\ell},\left(\breve{Q}_{j}-\breve{Q}_{j-1}\right) w_{\ell^{\prime}}\right\rangle_{L_{2}(\Gamma)} \\
& \lesssim \sum_{\ell, \ell^{\prime}=j_{0}}^{\infty} \sum_{j=j_{0}}^{\min \left\{\ell, \ell^{\prime}\right\}} 4^{s j} 4^{-t j}\left\|w_{\ell}\right\|\left\|_{t}\right\| w_{\ell^{\prime}}\left\|_{t} \lesssim \sum_{\ell, \ell^{\prime}=j_{0}}^{\infty} 4^{(s-t) \min \left\{\ell, \ell^{\prime}\right\}}\right\| w_{\ell}\|t\| w_{\ell^{\prime}} \|_{t} \\
& \lesssim \sum_{\ell, \ell^{\prime}=j_{0}}^{\infty} 4^{(s-t) \min \left\{\ell, \ell^{\prime}\right\}} 2^{(t-s)\left(\ell+\ell^{\prime}\right)}\left(2^{s \ell}\left\|w_{\ell}\right\|_{L_{2}(\Gamma)}\right)\left(2^{s \ell^{\prime}}\left\|w_{\ell^{\prime}}\right\|_{L_{2}(\Gamma)}\right) \\
& \lesssim \sum_{\ell=j_{0}}^{\infty} 4^{s \ell}\left\|w_{\ell}\right\|_{L_{2}(\Gamma)}^{2} \approx\|u\|_{s}^{2},
\end{aligned}
$$

where we have used that the infinite matrix $\left[2^{(s-t)\left(2 \min \left\{\ell, \ell^{\prime}\right\}-\ell-\ell^{\prime}\right)}\right]_{\ell, \ell^{\prime} \geq j_{0}}$ is bounded.

(VI) For $s \in[-\tilde{d}, \gamma)$, it holds that

$$
\|\cdot\|_{s} \lesssim 2^{s j}\|\cdot\|_{L_{2}(\Gamma)} \quad \text { on } \Im\left(\breve{Q}_{j}-\breve{Q}_{j-1}\right) .
$$

Indeed for $s>0$ this is just the Bernstein inequality (4.5), whereas for $s<0$ it is a consequence of the extended Bernstein inequality Lemma 4.2 and (A.2). Now let $s \in$ $(-\tilde{d}, \gamma)$, and let $\varepsilon>0$ be such that $s \pm \varepsilon \in[-\tilde{d}, \gamma)$. Then for any $\breve{w}_{j} \in \Im\left(\breve{Q}_{j}-\breve{Q}_{j-1}\right)$, with $\sum_{j=j_{0}}^{\infty} 4^{s j}\left\|\breve{w}_{j}\right\|_{L_{2}(\Gamma)}^{2}<\infty$, it holds that

$$
\begin{aligned}
& \left\|\sum_{j=j_{0}}^{\infty} \breve{w}_{j}\right\|_{s}^{2}=\sum_{j, j^{\prime}=j_{0}}^{\infty}\left\langle\left\langle\breve{w}_{j}, \breve{w}_{j^{\prime}}\right\rangle\right\rangle_{s} \lesssim \sum_{j=j_{0}}^{\infty} \sum_{j^{\prime} \geq j}^{\infty}\left\|\breve{w}_{j}\right\|_{s+\varepsilon}\left\|\breve{w}_{j^{\prime}}\right\|_{s-\varepsilon} \\
& \lesssim \sum_{j=j_{0}}^{\infty} \sum_{j^{\prime} \geq j}^{\infty} 2^{\varepsilon j} 2^{-\varepsilon j^{\prime}}\left(2^{s j}\left\|\breve{w}_{j}\right\|_{L_{2}(\Gamma)}\right)\left(2^{s j^{\prime}}\left\|\breve{w}_{j^{\prime}}\right\|_{L_{2}(\Gamma)}\right) \lesssim \sum_{j=j_{0}}^{\infty} 4^{s j}\left\|\breve{w}_{j}\right\|_{L_{2}(\Gamma)}^{2} .
\end{aligned}
$$


Since, when $s \in(t, \min \{\gamma, d\}),\left(\right.$ A.4) shows that $\sum_{j=j_{0}}^{\infty} 4^{s j}\left\|\breve{w}_{j}\right\|_{L_{2}(\Gamma)}^{2} \lesssim\left\|\sum_{j=j_{0}}^{\infty} \breve{w}_{j}\right\|_{s}^{2}$, and $\Phi_{j_{0}}$ is an $L_{2}(\Gamma)$-Riesz basis for $\Im\left(\breve{Q}_{j_{0}}\right)$, and for $j>j_{0}, \breve{\Psi}_{j-1}$ is a uniform $L_{2}(\Gamma)$ Riesz basis for $\Im\left(\breve{Q}_{j}-\breve{Q}_{j-1}\right)$, we conclude that $\Phi_{j_{0}} \cup \cup_{j \geq j_{0}} 2^{-s j} \breve{\Psi}_{j}$ is a Riesz system in $\mathcal{H}_{s}(\Gamma)$. Finally, since, as follows from Theorem 5.3, $\operatorname{clos}_{H_{s}(\Gamma)} \cup_{j \geq 0} \mathcal{S}\left(\Phi_{j}\right)=\mathcal{H}_{s}(\Gamma)$, we conclude it is even Riesz basis for this space, with which the proof of Theorem 6.1 is completed.

Acknowledgment. The author is indebted to H. Nguyen for the computation of the numerical results.

\section{REFERENCES}

[1] C. Canuto, A. Tabacco, and K. Urban. The wavelet element method part I: Construction and analysis. Appl. Comput. Harmon. Anal., 6:1-52, 1999.

[2] J.M. Carnicer, W. Dahmen, and J.M. Peña. Local decomposition of refinable spaces and wavelets. Appl. and Comp. Harm. Anal., 3:127-153, 1996.

[3] A. Cohen. Numerical Analysis of Wavelet Methods. Elsevier, Amsterdam, 2003.

[4] A. Cohen, W. Dahmen, and R. DeVore. Adaptive wavelet methods for elliptic operator equations - Convergence rates. Math. Comp, 70:27-75, 2001.

[5] A. Cohen, W. Dahmen, and R. DeVore. Adaptive wavelet methods II - Beyond the elliptic case. Found. Comput. Math., 2(3):203-245, 2002.

[6] A. Cohen, I. Daubechies, and J.C. Feauveau. Biorthogonal bases of compactly supported wavelets. Comm. Pur. Appl. Math., 45:485-560, 1992.

[7] A. Cohen and R. Masson. Wavelet adaptive method for second order elliptic problems: Boundary conditions and domain decomposition. Numer. Math., 86:193-238, 2000.

[8] W. Dahmen. Stability of multiscale transformations. J. Fourier Anal. Appl., 4:341-362, 1996.

[9] W. Dahmen. Wavelet and multiscale methods for operator equations. Acta Numer., 6:55-228, 1997.

[10] W. Dahmen, H. Harbrecht, and R. Schneider. Compression techniques for boundary integral equations - optimal complexity estimates. IGPM report, RWTH Aachen, June 2002. To appear in SIAM J. Numer. Anal.

[11] W. Dahmen, A. Kunoth, and K. Urban. Biorthogonal spline-wavelets on the interval - Stability and moment conditions. Appl. Comp. Harm. Anal., 6:132-196, 1999.

[12] W. Dahmen and R. Schneider. Composite wavelet bases for operator equations. Math. Comp., 68:1533-1567, 1999.

[13] W. Dahmen and R. Schneider. Wavelets on manifolds I: Construction and domain decomposition. SIAM J. Math. Anal., 31:184-230, 1999.

[14] W. Dahmen and R.P. Stevenson. Element-by-element construction of wavelets satisfying stability and moment conditions. SIAM J. Numer. Anal., 37(1):319-352, 1999.

[15] T. Gantumur, H. Harbrecht, and R.P. Stevenson. An optimal adaptive wavelet method without coarsening of the iterands. Technical Report 1325, Utrecht University, March 2005. To appear in Math. Comp.

[16] A. Kunoth and J. Sahner. Wavelets on manifolds: An optimized construction. SFB 611 Preprint 163, Universität Bonn, July 2004. To appear in Math. Comp.

[17] H. Nguyen. Finite element wavelets for solving partial differential equations. PhD thesis, Utrecht University, 2005.

[18] H. Nguyen and R.P. Stevenson. Finite element wavelets on manifolds. IMA J. Numer. Math, 23:149-173, 2003

[19] R. Schneider. Multiskalen- und Wavelet-Matrixkompression: Analysisbasierte Methoden zur Lösung großer vollbesetzter Gleigungssysteme. Habilitationsschrift, 1995. Advances in Numerical Mathematics. Teubner, Stuttgart, 1998.

[20] R.P. Stevenson. Stable three-point wavelet bases on general meshes. Numer. Math., 80:131-158, 1998.

[21] R.P. Stevenson. Locally supported, piecewise polynomial biorthogonal wavelets on non-uniform meshes. Constr. Approx., 19(4):477-508, 2003.

[22] R.P. Stevenson. On the compressibility of operators in wavelet coordinates. SIAM J. Math. Anal., 35(5):1110-1132, 2004.

[23] P.S. Vassilevski and J. Wang. Stabilizing the hierarchical basis by approximate wavelets, II: Implementation and numerical experiments. SIAM J. Sci. Comput., 20:490-514, 1999. 
[24] J. Xu. Iterative methods by space decomposition and subspace correction. SIAM Rev., 34:581613, 1992. 